Pure and Applied Mathematics Quarterly

Volume 8 , Number 2

(Special Issue: In honor of

F. Thomas Farrell and Lowell E. Jones, Part 2 of 2)

497-555, 2012

\title{
On The Classifying Space of The Family of Virtually Cyclic Subgroups
}

\author{
Wolfgang Lück and Michael Weiermann
}

\begin{abstract}
We study the minimal dimension of the classifying space of the family of virtually cyclic subgroups of a discrete group. We give a complete answer for instance if the group is virtually poly- $\mathbb{Z}$, word-hyperbolic or countable locally virtually cyclic. We give examples of groups for which the difference of the minimal dimensions of the classifying spaces of virtually cyclic and of finite subgroups is $-1,0$ and 1 , and show in many cases that no other values can occur.
\end{abstract}

Keywords: classifying spaces of families, dimensions, virtually cyclic subgroups.

\section{Introduction}

In this paper, we study the classifying spaces $\underline{\underline{E}} G=E_{\mathcal{V C Y}}(G)$ of the family of virtually cyclic subgroups of a group $G$. We are mainly interested in the minimal dimension $\operatorname{hdim}^{G}(\underline{\underline{E}} G)$ that a $G-C W$-model for $\underline{\underline{E}} G$ can have. The classifying space for proper $G$-actions $\underline{E} G=E_{\mathcal{F} \mathcal{I N}}(G)$ has already been studied 
intensively in the literature. The spaces $\underline{E} G$ and $\underline{\underline{E}} G$ appear in the source of the assembly maps whose bijectivity is predicted by the Baum-Connes conjecture and the Farrell-Jones conjecture, respectively. Hence the analysis of $\underline{E} G$ and $\underline{\underline{E}} G$ is important for computations of $K$ - and $L$-groups of reduced group $C^{*}$-algebras and of group rings (see Section 6). These spaces can also be viewed as invariants of $G$, and one would like to understand what properties of $G$ are reflected in the geometry and homotopy theoretic properties of $\underline{E} G$ and $\underline{\underline{E}} G$.

The main results of this manuscript aim at the computation of the homotopy dimension $\operatorname{hdim}^{G}(\underline{\underline{E}} G)$ (see Definition 4.1), which is much more difficult than in the case of $\operatorname{hdim}^{G}(\underline{E} G)$. It has lead to some (at least for us) surprising phenomenons, already for some nice groups which can be described explicitly and have interesting geometry.

After briefly recalling the notion of $\underline{E} G$ and $\underline{\underline{E}} G$ in Section 1, we investigate in Section 2 how one can build $E_{\mathcal{G}}(G)$ out of $\overline{E_{\mathcal{F}}}(G)$ for families $\mathcal{F} \subseteq \mathcal{G}$. We construct a $G$-pushout

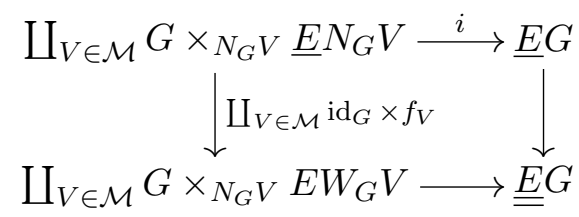

for a certain set $\mathcal{M}$ of maximal virtually cyclic subgroups of $G$, provided that $G$ satisfies the condition $\left(M_{\mathcal{F} \mathcal{I N} \subseteq \mathcal{V} \mathcal{C Y}}\right)$ that every infinite virtually cyclic subgroup $H$ is contained in a unique maximal infinite virtually cyclic subgroup $H_{\max }$.

For a subgroup $H \subseteq G$, we denote by $N_{G} H:=\left\{g \in G \mid g H g^{-1}=H\right\}$ its normalizer and put $W_{G} H:=N_{G} H / H$. We will say that $G$ satisfies $\left(N M_{\mathcal{F} \mathcal{I N} \subseteq \mathcal{V} \mathcal{Y} Y}\right)$ if it satisfies $\left(M_{\mathcal{F} \mathcal{I N} \subseteq \mathcal{V} \mathcal{Y} Y}\right)$ and $N_{G} V=V$, i.e., $W_{G} V=\{1\}$, holds for all $V \in \mathcal{M}$.

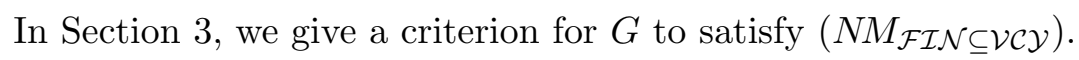

Section 4 is devoted to the construction of models for $\underline{E} G$ and $\underline{E} G$ from models for $\underline{\underline{E}} G_{i}$ and $\underline{E} G_{i}$, respectively, if $G$ is the directed union of the directed system $\left\{G_{i} \mid i \in I\right\}$ of subgroups.

In Section 5 , we prove various results concerning $\operatorname{hdim}^{G}(\underline{\underline{E}} G)$ and give some examples. In Subsection 5.1, we prove

$$
\operatorname{hdim}^{G}(\underline{E} G) \leq 1+\operatorname{hdim}^{G}(\underline{\underline{E}} G) .
$$


While one knows that $\operatorname{hdim}^{G \times H}(\underline{E}(G \times H)) \leq \operatorname{hdim}^{G}(\underline{E} G)+\operatorname{hdim}^{H}(\underline{E} H)$, we prove that

$$
\operatorname{hdim}^{G \times H}(\underline{\underline{E}}(G \times H)) \leq \operatorname{hdim}^{G}(\underline{\underline{E}} G)+\operatorname{hdim}^{H}(\underline{\underline{E}} H)+3
$$

and give examples showing that this inequality cannot be improved in general.

In Subsection 5.2, we show

$$
\operatorname{hdim}^{G}(\underline{\underline{E}} G) \begin{cases}=\operatorname{hdim}^{G}(\underline{E} G) & \text { if } \operatorname{hdim}^{G}(\underline{E} G) \geq 2 ; \\ \leq 2 & \text { if } \operatorname{hdim}^{G}(\underline{E} G) \leq 1\end{cases}
$$

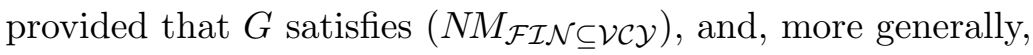

$$
\operatorname{hdim}^{G}(\underline{\underline{E}} G) \leq \operatorname{hdim}^{G}(\underline{E} G)+1
$$

provided that $G$ satisfies $\left(M_{\mathcal{F} \mathcal{I N} \subseteq \mathcal{V} \mathcal{Y} Y}\right)$. This implies that the fundamental group $\pi$ of a closed hyperbolic closed manifold $M$ satisfies

$$
\operatorname{vcd}(\pi)=\operatorname{dim}(N)=\operatorname{hdim}^{\pi}(\underline{E} \pi)=\operatorname{hdim}^{\pi}(\underline{\underline{E}} \pi),
$$

yielding a counterexample to a conjecture due to Connolly-Fehrmann-Hartglass.

In Subsection 5.3, a complete computation of $\operatorname{him}^{G}(\underline{\underline{E}} G)$ is presented for virtually poly- $\mathbb{Z}$ groups. This leads to some interesting examples in Subsection 5.4. For instance, we construct, for $k=-1,0,1$, automorphisms $f_{k}$ : Hei $\rightarrow$ Hei of the three-dimensional Heisenberg group Hei such that

$$
\operatorname{hdim}^{\text {Hei }_{f_{k}} \mathbb{Z}}\left(\underline{\underline{E}}\left(\text { Hei }_{f_{k}} \mathbb{Z}\right)\right)=4+k .
$$

Notice that hdim ${ }^{\text {Hei } \rtimes_{f_{k}} \mathbb{Z}}\left(\underline{E}\left(\right.\right.$ Hei $\left.\left.\rtimes_{f} \mathbb{Z}\right)\right)=\operatorname{cd}\left(\right.$ Hei $\left.\rtimes_{f} \mathbb{Z}\right)=4$ holds for any automorphism $f:$ Hei $\rightarrow$ Hei.

In Subsection 5.5, we briefly investigate $\operatorname{hdim}^{G}\left(E_{\mathcal{S F G}}(G)\right)$, where $\mathcal{S F \mathcal { G }}$ is the family of subgroups of $G$ which are contained in some finitely generated subgroup. After that, in Subsection 5.6, we deal with groups for which the values of hdim ${ }^{G}(\underline{E} G)$ and $\operatorname{hdim}^{G}(\underline{E} G)$ are small. We show that a countable infinite group $G$ is locally finite if and only if $\operatorname{hdim}^{G}(\underline{E} G)=\operatorname{hdim}^{G}(\underline{\underline{E}} G)=1$.

Finally, we explain in Section 6 how the models we construct for $\underline{\underline{E}} G$ can be used to obtain information about the relative equivariant homology group that 
appears in the source of the assembly map in the Farrell-Jones conjecture for algebraic $K$-theory.

All the results in this paper raise the following question:

For which groups $G$ is it true that

$$
\operatorname{hdim}^{G}(\underline{E} G)-1 \leq \operatorname{hdim}^{G}(\underline{\underline{E}} G) \leq \operatorname{hdim}^{G}(\underline{E} G)+1 \quad ?
$$

We have no example of a group for which the above inequality is not true.

In this paper, we are not dealing with the question whether there is a finite $G$ $C W$-model for $\underline{\underline{E}} G$. Namely, there is no known counterexample to the conjecture (see [10, Conjecture 1]) that a group $G$ possessing a finite $G$ - $C W$-model for $\underline{\underline{E}} G$ is virtually cyclic.

The authors wishes to thank the referee for his detailed and valuable report.

The paper was supported by the Sonderforschungsbereich 478 - Geometrische Strukturen in der Mathematik - the Max-Planck-Forschungspreis and the LeibnizPreis of the first author. Parts of this paper have already appeared in the Ph.D. thesis of the second author.

\section{Classifying Spaces for Families}

We briefly recall the notions of a family of subgroups and the associated classifying spaces. For more information, we refer for instance to the survey article [16].

A family $\mathcal{F}$ of subgroups of $G$ is a set of subgroups of $G$ which is closed under conjugation and taking subgroups. Examples for $\mathcal{F}$ are

$$
\begin{aligned}
\{1\} & =\{\text { trivial subgroup }\} \\
\mathcal{F} \mathcal{I N} & =\{\text { finite subgroups }\} \\
\mathcal{V C Y} & =\{\text { virtually cyclic subgroups }\} \\
\mathcal{S} \mathcal{F} \mathcal{G} & =\{\text { subgroups of finitely generated subgroups }\} \\
\mathcal{A L} \mathcal{L} & =\{\text { all subgroups }\}
\end{aligned}
$$


Let $\mathcal{F}$ be a family of subgroups of $G$. A model for the classifying space $E_{\mathcal{F}}(G)$ of the family $\mathcal{F}$ is a $G$ - $C W$-complex $X$ all of whose isotropy groups belong to $\mathcal{F}$ and such that for any $G-C W$-complex $Y$ whose isotropy groups belong to $\mathcal{F}$ there is precisely one $G$-map $Y \rightarrow X$ up to $G$-homotopy. In other words, $X$ is a terminal object in the $G$-homotopy category of $G$-CW-complexes whose isotropy groups belong to $\mathcal{F}$. In particular, two models for $E_{\mathcal{F}}(G)$ are $G$-homotopy equivalent, and for two families $\mathcal{F}_{0} \subseteq \mathcal{F}_{1}$ there is precisely one $G$-map $E_{\mathcal{F}_{0}}(G) \rightarrow E_{\mathcal{F}_{1}}(G)$ up to $G$-homotopy. There exists a model for $E_{\mathcal{F}}(G)$ for any group $G$ and any family $\mathcal{F}$ of subgroups.

A $G$-CW-complex $X$ is a model for $E_{\mathcal{F}}(G)$ if and only if the $H$-fixed point set $X^{H}$ is contractible for $H \in \mathcal{F}$ and is empty for $H \notin \mathcal{F}$.

We abbreviate $\underline{E} G:=E_{\mathcal{F} \mathcal{I N}}(G)$ and call it the universal $G$-CW-complex for proper $G$-actions. We also abbreviate $\underline{\underline{E}} G:=E_{\mathcal{V C Y}}(G)$.

A model for $E_{\mathcal{A L L}}(G)$ is $G / G$. A model for $E_{\{1\}}(G)$ is the same as a model for $E G$, which denotes the total space of the universal $G$-principal bundle $E G \rightarrow B G$. Our interest in these notes concerns the spaces $\underline{\underline{E}} G$.

\section{Passing to larger families}

In this section, we explain in general how one can construct a model for $E_{\mathcal{G}}(G)$ from $E_{\mathcal{F}}(G)$ if $\mathcal{F}$ and $\mathcal{G}$ are families of subgroups of the group $G$ with $\mathcal{F} \subseteq \mathcal{G}$. Let $\sim$ be an equivalence relation on $\mathcal{G} \backslash \mathcal{F}$ with the properties:

- If $H, K \in \mathcal{G} \backslash \mathcal{F}$ with $H \subseteq K$, then $H \sim K$;

- If $H, K \in \mathcal{G} \backslash \mathcal{F}$ and $g \in G$, then $H \sim K \Leftrightarrow g H g^{-1} \sim g K g^{-1}$.

Let $[\mathcal{G} \backslash \mathcal{F}]$ be the set of equivalence classes of $\sim$. Denote by $[H] \in[\mathcal{G} \backslash \mathcal{F}]$ the equivalence class of $H \in \mathcal{G} \backslash \mathcal{F}$, and define the subgroup

$$
N_{G}[H]:=\left\{g \in G \mid\left[g^{-1} H g\right]=[H]\right\}
$$

of $G$. Then $N_{G}[H]$ is the isotropy group of $[H]$ under the $G$-action on $[\mathcal{G} \backslash \mathcal{F}]$ induced by conjugation. Define a family of subgroups of $N_{G}[H]$ by

$$
\mathcal{G}[H]:=\left\{K \subseteq N_{G}[H] \mid K \in \mathcal{G} \backslash \mathcal{F},[K]=[H]\right\} \cup\left(\mathcal{F} \cap N_{G}[H]\right),
$$


where $\mathcal{F} \cap N_{G}[H]$ consists of all the subgroups of $N_{G}[H]$ belonging to $\mathcal{F}$.

Definition 2.2 (Equivalence relation on $\mathcal{V C Y} \backslash \mathcal{F} \mathcal{I N}$ ). If $\mathcal{G}=\mathcal{V C Y}$ and $\mathcal{F}=$ $\mathcal{F} \mathcal{I N}$, we will take for the equivalence relation $\sim$

$$
V \sim W \Leftrightarrow|V \cap W|=\infty
$$

Theorem 2.3 (Constructing models from given ones). Let $\mathcal{F} \subseteq \mathcal{G}$ and $\sim$ be as above such that properties (2.1) hold. Let I be a complete system of representatives $[H]$ of the $G$-orbits in $[\mathcal{G} \backslash \mathcal{F}]$ under the $G$-action coming from conjugation. Choose arbitrary $N_{G}[H]-C W$-models for $E_{\mathcal{F} \cap N_{G}[H]}\left(N_{G}[H]\right)$ and $E_{\mathcal{G}[H]}\left(N_{G}[H]\right)$, and an arbitrary $G-C W$-model for $E_{\mathcal{F}}(G)$. Define a $G$-CW-complex $X$ by the cellular $G$-pushout

$$
\begin{aligned}
& \coprod_{[H] \in I} G \times_{N_{G}[H]} E_{\mathcal{F} \cap N_{G}[H]}\left(N_{G}[H]\right) \stackrel{i}{\longrightarrow} E_{\mathcal{F}}(G)
\end{aligned}
$$

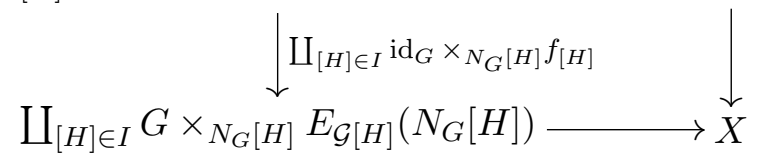

such that $f_{[H]}$ is a cellular $N_{G}[H]$-map for every $[H] \in I$ and $i$ is an inclusion of $G$-CW-complexes, or such that every map $f_{[H]}$ is an inclusion of $N_{G}[H]-C W$ complexes for every $[H] \in I$ and $i$ is a cellular $G$-map.

Then $X$ is a model for $E_{\mathcal{G}}(G)$.

Proof. We have to show that $X^{K}$ is contractible if $K$ belongs to $\mathcal{G}$, and that it is empty, otherwise. For $[H] \in I$, let $s_{[H]}: G / N_{G}[H] \rightarrow G$ be a set-theoretic section of the projection $G \rightarrow G / N_{G}[H]$. Let $K \subseteq G$ be a subgroup. Taking $K$ fixed points in the above $G$-pushout yields, up to homeomorphism, the following pushout

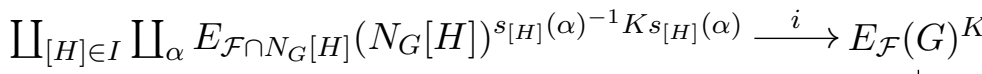

$$
\begin{aligned}
& \amalg_{[H] \in I} \amalg_{\alpha} f_{[H], \alpha} \downarrow \\
& \coprod_{[H] \in I} \coprod_{\alpha} E_{\mathcal{G}[H]}\left(N_{G}[H]\right)^{s_{[H]}(\alpha)^{-1} K s_{[H]}(\alpha)} \longrightarrow \underset{X^{K}}{\downarrow}
\end{aligned}
$$

in which one of the maps starting from the left upper corner is a cofibration and $\alpha$ runs through $\left\{\alpha \in G / N_{G}[H] \mid s_{[H]}(\alpha)^{-1} K s_{[H]}(\alpha) \subseteq N_{G}[H]\right\}$. 
Assume first that $K \notin \mathcal{G}$. Then the entries in the upper row and the lower left entry of (2.4) are clearly empty. Hence, in this case $X^{K}$ is empty.

If $K \in \mathcal{G} \backslash \mathcal{F}$, the entries in the upper row of (2.4) are again empty, so in order to show that $X^{K}$ is contractible, we must show that the lower left entry of (2.4) is contractible. Note that the space $E_{\mathcal{G}[H]}\left(N_{G}[H]\right)^{s_{[H]}}(\alpha)^{-1} K s_{[H]}(\alpha)$ is nonempty if and only if $s_{[H]}(\alpha)^{-1} K s_{[H]}(\alpha)$ belongs to the family $\mathcal{G}[H]$ of subgroups of $N_{G}[H]$, which is equivalent to the condition that $\left[s_{[H]}(\alpha)^{-1} K s_{[H]}(\alpha)\right]=[H]$ and $s_{[H]}(\alpha)^{-1} K s_{[H]}(\alpha) \subseteq N_{G}[H]$. Now, there is precisely one $\left[H_{K}\right] \in I$ for which there exists $g_{K} \in G$ with the property that $\left[g_{K}^{-1} K g_{K}\right]=\left[H_{K}\right]$. An element $g \in G$ satisfies $\left[g^{-1} K g\right]=\left[H_{K}\right]$ if and only if $g_{K}^{-1} g \in N_{G}\left[H_{K}\right]$. Moreover, $L \subseteq N_{G}[H]$ always holds if $L \in \mathcal{G} \backslash \mathcal{F}$ is such that $[L]=[H]$. We conclude from these observations that $E_{\mathcal{G}[H]}\left(N_{G}[H]\right)^{s_{[H]}(\alpha)^{-1} K s_{[H]}(\alpha)}$ is non-empty if and only if $[H]=\left[H_{K}\right]$ and $\alpha=g_{K} N_{G}\left[H_{K}\right]$. Furthermore, it is contractible in this case. This implies that the lower left entry of (2.4) is contractible.

Finally, if $K \in \mathcal{F}$, then the upper right entry of (2.4) is contractible. This implies that the same will hold for $X^{K}$ if we can show that all the maps $f_{[H], \alpha}$ are homotopy equivalences. But this is clear since the source and target spaces of the $f_{[H], \alpha}$ are contractible.

Remark 2.5 (Dimension of the model constructed for $E_{\mathcal{G}}(G)$ ). A $G$-pushout as described in Theorem 2.3 always exists. To be more precise, the maps $i$ and $f_{[H]}$ exist and are unique up to equivariant homotopy equivalence due to the universal property of the classifying spaces $E_{\mathcal{F}}(G)$ and $E_{\mathcal{G}[H]}\left(N_{G}[H]\right)$. Moreover, because of the equivariant cellular approximation theorem (see for instance [13, Theorem 2.1 on page 32]), these maps can be assumed to be cellular. Finally, one can replace a cellular $G$-map $f: X \rightarrow Y$ by the canonical inclusion $j: X \rightarrow \operatorname{cyl}(f)$ into its mapping cylinder. Then the canonical projection $p: \operatorname{cyl}(f) \rightarrow Y$ is a $G$ homotopy equivalence such that $\operatorname{pr} \circ i=f$.

Note that the dimension of $\operatorname{cyl}(f)$ is $\max \{1+\operatorname{dim}(X), \operatorname{dim}(Y)\}$. This means that we we get the following conclusion from Theorem 2.3 which we will often use: There exists an $n$-dimensional $G$ - $C W$-model for $E_{\mathcal{G}}(G)$ if there exists an $n$-dimensional $G$ - $C W$-model for $E_{\mathcal{F}}(G)$ and, for every $H \in I$, an $(n-$ 1)-dimensional $N_{G}[H]$-CW-model for $E_{\mathcal{F} \cap N_{G}[H]}\left(N_{G}[H]\right)$ and an $n$-dimensional 
$N_{G}[H]$-CW-model for $E_{\mathcal{G}[H]}\left(N_{G}[H]\right)$.

Example 2.6. In general $N_{G}[V]$, cannot be written as a normalizer $N_{G} W$ for some $W \in[V]$ in the case $\mathcal{G}=\mathcal{V} \mathcal{C Y}, \mathcal{F}=\mathcal{F} \mathcal{I N}$ and $\sim$ as defined in Definition 2.2.

For instance, let $p$ be a prime number. Let $\mathbb{Z}[1 / p]$ be the subgroup of $\mathbb{Q}$ consisting of rational numbers $x \in \mathbb{Q}$ for which $p^{n} \cdot x \in \mathbb{Z}$ for some positive integer $n$. This is the directed union $\bigcup_{n \in \mathbb{N}} p^{-n} \cdot \mathbb{Z}$. Let $p \cdot \operatorname{id}: \mathbb{Z}[1 / p] \rightarrow \mathbb{Z}[1 / p]$ be the automorphism given by multiplication with $p$, and define $G$ to be the semidirect product $\mathbb{Z}[1 / p] \rtimes_{p \text {.id }} \mathbb{Z}$. Let $C$ be the cyclic subgroup of $\mathbb{Z}[1 / p]$ generated by 1 . Then $N_{G}[C]=G$ but $N_{G} V=\mathbb{Z}[1 / p]$ for every $V \in[C]$.

Notation 2.7. Let $\mathcal{F} \subseteq \mathcal{G}$ be families of subgroups of a group $G$.

We shall say that $G$ satisfies $\left(M_{\mathcal{F} \subseteq \mathcal{G}}\right)$ if every subgroup $H \in \mathcal{G} \backslash \mathcal{F}$ is contained in a unique $H_{\max } \in \mathcal{G} \backslash \mathcal{F}$ which is maximal in $\mathcal{G} \backslash \mathcal{F}$, i.e., $H_{\max } \subseteq K$ for $K \in \mathcal{G} \backslash \mathcal{F}$ implies $H_{\max }=K$.

We shall say that $G$ satisfies $\left(N M_{\mathcal{F} \subseteq \mathcal{G}}\right)$ if $M$ satisfies $\left(M_{\mathcal{F} \subseteq \mathcal{G}}\right)$ and every maximal element $M \in \mathcal{G} \backslash \mathcal{F}$ equals its normalizer, i.e., $N_{G} M=M$ or, equivalently, $W_{G} M=\{1\}$.

Examples of groups satisfying $\left(N_{\mathcal{F} \mathcal{I} \mathcal{N} \subseteq \mathcal{V} \mathcal{Y} Y}\right)$ will be given in Section 3. In [7, page 101], Davis-Lück discuss examples of groups satisfying $\left(M_{\{1\} \subseteq \mathcal{F} \mathcal{I N}}\right)$ are discussed. The following will be a consequence of Theorem 2.3:

Corollary 2.8. Let $\mathcal{F} \subseteq \mathcal{G}$ be families of subgroups of a group $G$ which satisfies $\left(M_{\mathcal{F} \subseteq \mathcal{G}}\right)$. Let $\mathcal{M}$ be a complete system of representatives of the conjugacy classes of subgroups in $\mathcal{G} \backslash \mathcal{F}$ which are maximal in $\mathcal{G} \backslash \mathcal{F}$. Let $\mathcal{S U B}(M)$ be the family of subgroups of $M$. Consider a cellular $G$-pushout

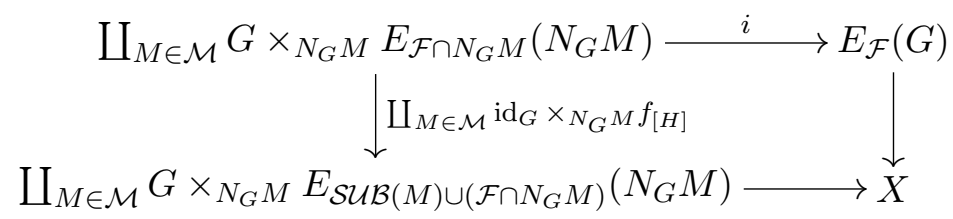

such that $f_{[H]}$ is a cellular $N_{G}[H]$-map for every $[H] \in I$ and $i$ is an inclusion of $G$-CW-complexes, or such that $f_{[H]}$ is an inclusion of $N_{G}[H]-C W$-complexes for every $[H] \in I$ and $i$ is a cellular $G$-map.

Then $X$ is a model for $E_{\mathcal{G}}(G)$. 
Proof. Let $H_{\text {max }}$ denote the unique maximal element in $\mathcal{G} \backslash \mathcal{F}$ which contains $H \in \mathcal{G} \backslash \mathcal{F}$. We use the equivalence relation on $\mathcal{G} \backslash \mathcal{F}$ given by $H \sim K \Leftrightarrow H_{\max }=$ $K_{\text {max }}$. Then, for $M \in \mathcal{M}$, the family $\mathcal{G}[M]$ is just $\{K \in \mathcal{G} \mid K \subseteq M$ or $K \in \mathcal{F}\}$. Now apply Theorem 2.3 .

Remark 2.9. If $G$ satisfies $\left(M_{\mathcal{F} \mathcal{I N} \subseteq \mathcal{V} \mathcal{C Y})}\right)$, then the equivalence relation $\sim$ defined in Definition 2.2 agrees with the equivalence relation $H \sim K \Leftrightarrow H_{\max }=K_{\max }$ appearing in the proof of Corollary 2.8 above.

Corollary 2.10. Let $G$ be a group satisfying $\left(M_{\{1\} \subseteq \mathcal{F} \mathcal{I N}}\right)$ or $\left(M_{\mathcal{F} \mathcal{I N} \subseteq \mathcal{V} C \mathcal{Y}}\right)$ respectively. We denote by $\mathcal{M}$ a complete system of representatives of the conjugacy classes of maximal finite subgroups $F \subseteq G$ or of maximal infinite virtually cyclic subgroups $V \subseteq G$ respectively. Consider the cellular $G$-pushouts

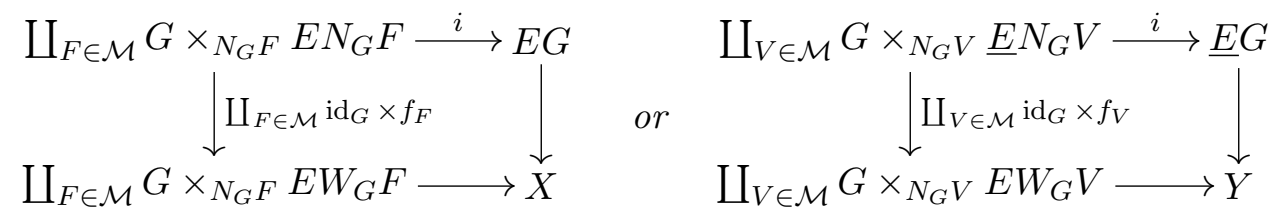

where $E W_{G} H$ is viewed as an $N_{G} H-C W$-complex by restricting with the projection $N_{G} H \rightarrow W_{G} H$ for $H \subseteq G$, the maps starting from the left upper corner are cellular and one of them is an inclusion of $G$-CW-complexes.

Then $X$ is a model for $\underline{E} G$ or $Y$ is a model for $\underline{\underline{E}} G$ respectively.

Proof. This follows from Corollary 2.8 and the following facts. A model for $E_{\mathcal{S U B}(H)}\left(N_{G} H\right)$ is given by $E W_{G} H$ considered as an $N_{G} H$ - $C W$-complex by restricting with the projection $N_{G} H \rightarrow W_{G} H$ for every $H \subseteq G$. Furthermore, we obviously have $\{1\} \cap N_{G} F \subseteq \mathcal{S U B}(F)$ for every maximal finite subgroup $F$, whereas $\mathcal{F} \mathcal{I N} \cap N_{G} V \subseteq \mathcal{S U B}(V)$ for every maximal infinite virtually cyclic subgroup $V \subseteq G$ because $W_{G} V$ is torsionfree (if it were not, then the preimage of a non-trivial finite subgroup of $W_{G} V$ under the projection $N_{G} V \rightarrow W_{G} V$ would be virtually cyclic and strictly containing $V$, contradicting its maximality).

As a special case of Corollary 2.10, we get

Corollary 2.11. Let $G$ be a group satisfying $\left(N M_{\{1\} \subseteq \mathcal{F} \mathcal{I N})}\right.$ or $\left(N M_{\mathcal{F} \mathcal{I N} \subseteq \mathcal{V} \mathcal{Y} Y}\right)$ respectively. Let $\mathcal{M}$ be a complete system of representatives of the conjugacy 
classes of maximal finite subgroups $F \subseteq G$ or of maximal infinite virtually cyclic subgroups $V \subseteq G$ respectively. Consider the cellular $G$-pushouts

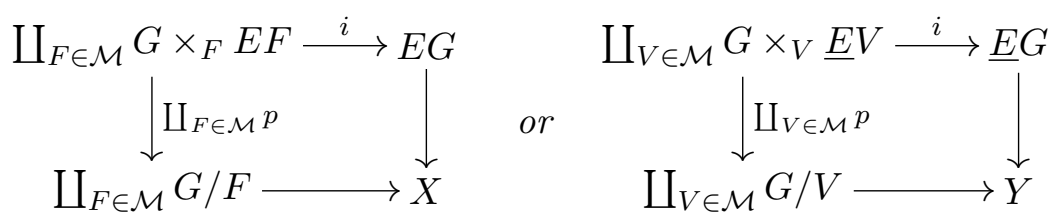

where $i$ is an inclusion of $G$-CW-complexes and $p$ is the obvious projection.

Then $X$ is a model for $\underline{E} G$ or $Y$ is a model for $\underline{\underline{E}} G$ respectively.

\section{A Class of Groups satisfying $\left(N_{\mathcal{F} \mathcal{I N} \subseteq \mathcal{V} \mathcal{C Y}}\right)$}

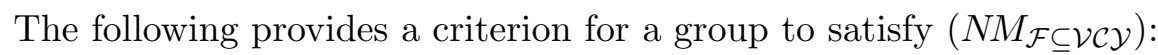

Theorem 3.1. Suppose that the group $G$ satisfies the following two conditions:

- Every infinite cyclic subgroup $C \subseteq G$ has finite index $\left[C_{G} C: C\right]$ in its centralizer;

- Every ascending chain $H_{1} \subseteq H_{2} \subseteq$.. of finite subgroups of $G$ becomes stationary, i.e., there is an $n_{0} \in \mathbb{N}$ such that $H_{n}=H_{n_{0}}$ for all $n \geq n_{0}$.

Then every infinite virtually cyclic subgroup $V \subseteq G$ is contained in a unique maximal virtually cyclic subgroup $V_{\max } \subseteq G$. Moreover, $V_{\max }$ is equal to its normalizer $N_{G}\left(V_{\max }\right)$, and

$$
V_{\max }=\bigcup_{C \subseteq V} N_{G} C
$$

where the union is over all infinite cyclic subgroups $C \subseteq V$ that are normal in $V$.

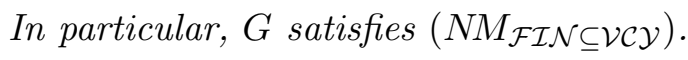

Proof. We fix an infinite virtually cyclic subgroup $V \subseteq G$ and define $V_{\max }:=$ $\bigcup_{C \subseteq V} N_{G} C$, where the union is over all infinite cyclic subgroups $C \subseteq V$ that are normal in $V$. The collection of all such subgroups of $V$ is countable, and we denote it by $\left\{C_{n}\right\}_{n \in \mathbb{N}}$. Since every index $\left[V: C_{n}\right]$ is finite, $\left[V: C_{0} \cap \ldots \cap C_{n}\right]$ 
must also be finite for $n \in \mathbb{N}$. Thus, if we set $Z_{n}:=C_{0} \cap \ldots \cap C_{n}$, then $Z_{n} \subseteq C_{n}$, and $Z_{0} \supseteq Z_{1} \supseteq \ldots$ is a descending chain of infinite cyclic subgroups of $V$ that are normal in $V$. If $C^{\prime} \subseteq C$ are two infinite cyclic subgroups of $G$, then $N_{G} C \subseteq N_{G} C^{\prime}$ because $C^{\prime}$ is a characteristic subgroup of $C$. It follows in our situation that $N_{G} C_{n} \subseteq N_{G} Z_{n}$, which implies

$$
\bigcup_{n=0}^{\infty} N_{G} C_{n}=\bigcup_{n=0}^{\infty} N_{G} Z_{n} .
$$

Furthermore, $N_{G} Z_{0} \subseteq N_{G} Z_{1} \subseteq \ldots$ is an ascending chain, which becomes stationary by the following argument. Namely, we can estimate

$$
\left[N_{G} Z_{n}: N_{G} Z_{0}\right] \leq\left[N_{G} Z_{n}: C_{G} Z_{0}\right]=\left[N_{G} Z_{n}: C_{G} Z_{n}\right] \cdot\left[C_{G} Z_{n}: C_{G} Z_{0}\right],
$$

and the first factor on the right is not greater than 2 since there is an injection $N_{G} Z_{n} / C_{G} Z_{n} \rightarrow \operatorname{aut}\left(Z_{n}\right)$, whereas the second does not exceed an appropriate constant as we will show in Lemma 3.3 below. Hence, it follows from (3.2) that $V_{\max }=N_{G} Z_{n}$ for all $n \geq n_{0}$ if $n_{0} \in \mathbb{N}$ is sufficiently large. In other words, we can record that this construction yields, for any infinite cyclic subgroup $C \subseteq V$ that is normal in $V$, an infinite cyclic subgroup $Z \subseteq C$ that is normal in $V$ such that for all infinite cyclic subgroups $Z^{\prime} \subseteq Z$ we have $V_{\max }=N_{G} Z^{\prime}$.

Using the equality $V_{\max }=N_{G} Z_{n_{0}}$, it is now obvious that $V_{\max }$ is virtually cyclic since the finite index subgroup $C_{G} Z_{n_{0}}$ is so due to the assumption imposed on $G$. In addition, $V \subseteq V_{\max }$ since $Z_{n_{0}}$ is normal in $V$. Now suppose $W \subseteq G$ is an infinite virtually cyclic subgroup such that $V \subseteq W$. We claim that $V_{\max }=W_{\max }$. In order to prove this, let $Z_{W} \subseteq W$ be an infinite cyclic subgroup that is normal in $W$ such that $W_{\max }=N_{G} Z_{W}^{\prime}$ holds for all infinite cyclic subgroups $Z_{W}^{\prime} \subseteq Z_{W}$. Then $Z_{W} \cap V$ is a finite index subgroup of $W$ and so is an infinite cyclic subgroup of $V$ that is normal in $V$. As we have already seen, there is an infinite cyclic subgroup $Z_{V} \subseteq Z_{W} \cap V$ that is normal in $V$ such that $V_{\max }=N_{G} Z_{V}^{\prime}$ holds for all infinite cyclic subgroups $Z_{V}^{\prime} \subseteq Z_{V}$. Since $V$ has finite index in $W$ and $Z_{V}$ is normal in $V$, the intersection $Z_{V}^{\prime \prime}$ of all the conjugates of $Z_{V}$ by elements in $W$ has finite index in $Z_{V}$ and therefore is an infinite cyclic subgroup of $Z_{V}$. This implies $V_{\max }=N_{G} Z_{V}^{\prime \prime}=W_{\max }$. From this we can deduce immediately that $V_{\max }$ is indeed maximal among virtually cyclic subgroups of $G$ containing $V$ and that it is uniquely determined by this property. 
Finally, we will show that $N_{G} V_{\max }$ is virtually cyclic, so that it is equal to $V_{\max }$. Let $C \subseteq V_{\max }$ be infinite cyclic. Since $C$ has finite index in $V_{\max }$ and $V_{\max }$ contains only finitely many subgroups of index $\left[V_{\max }: C\right]$, the group $D$ which we define as the intersection of all conjugates of $C$ in $N_{G} V_{\max }$ has finite index in $V_{\max }$ and is therefore infinite cyclic as well. Obviously, $D$ is normal in $N_{G} V_{\max }$, so $N_{G} V_{\max } \subseteq N_{G} D$ holds, the latter being virtually cyclic because the finite index subgroup $C_{G} D$ is so by assumption. Hence Theorem 3.1 is proven as soon as we have finished the proof of the next lemma.

Lemma 3.3. There exists a natural number $N$ such that for all $n \in \mathbb{N}$ we have

$$
\left[C_{G} Z_{n}: C_{G} Z_{0}\right] \leq N
$$

Proof. Since the center of the virtually cyclic group $C_{G} Z_{n}$ contains $Z_{n}$ and hence is infinite, $C_{G} Z_{n}$ is of type I, i.e., possesses an epimorphism onto $\mathbb{Z}$. Denoting by $T_{n}$ the torsion subgroup of $H_{1}\left(C_{G} Z_{n}\right)$, it then follows that $H_{1}\left(C_{G} Z_{n}\right) / T_{n}$ is infinite cyclic. Let $p_{n}: C_{G} Z_{n} \rightarrow H_{1}\left(C_{G} Z_{n}\right) \rightarrow H_{1}\left(C_{G} Z_{n}\right) / T_{n}$ be the canonical projection, and let $c_{n} \in C_{G} Z_{n}$ be such that $p_{n}\left(c_{n}\right)$ is a generator.

Consider the commutative diagram

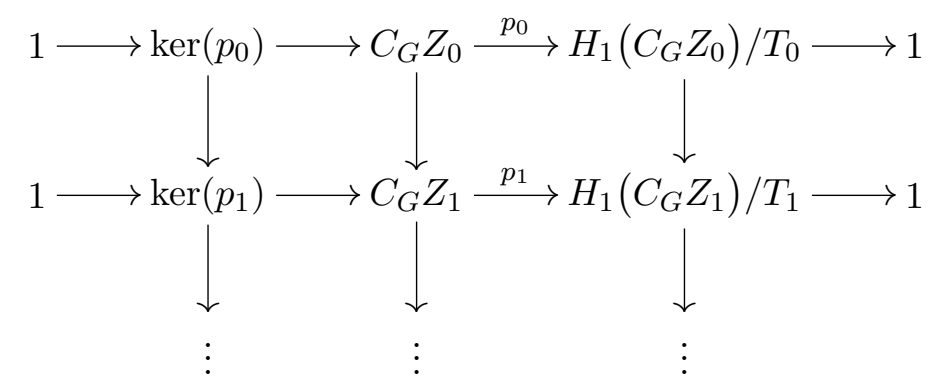

which has exact rows and in which all the vertical arrows in the first and second column are inclusions and all vertical arrows in the third column are induced by the obvious inclusions. They, too, are injective because $\operatorname{ker}\left(p_{n}\right)$ is finite and $H_{1}\left(C_{G} Z_{n}\right) / T_{n}$ is infinite cyclic for $n \in \mathbb{N}$. Since all the $\operatorname{ker}\left(p_{n}\right)$ are finite, $\bigcup_{n=0}^{\infty} \operatorname{ker}\left(p_{n}\right)$ is again finite, say of order $a \in \mathbb{N}$, by the assumption on $G$. Then in particular $\left[\operatorname{ker}\left(p_{n}\right): \operatorname{ker}\left(p_{0}\right)\right] \leq a$ for $n \in \mathbb{N}$, so $\left\{\left[C_{G} Z_{n}: C_{G} Z_{0}\right] \mid n \in \mathbb{N}\right\}$ will be bounded if we can show that the index of $H_{1}\left(C_{G} Z_{0}\right) / T_{0}$ in $H_{1}\left(C_{G} Z_{n}\right) / T_{n}$ is less than a constant which does not depend on $n \in \mathbb{N}$. 
In order to construct such a constant, let $r_{n} \in \mathbb{Z}$ be such that $p_{0}\left(c_{0}\right)$ is mapped to $p_{n}\left(c_{n}\right)^{r_{n}}$ under the inclusions in the above diagram. Then, by exactness, there is a $k_{n} \in \operatorname{ker}\left(p_{n}\right)$ such that $c_{0}=k_{n} c_{n}^{r_{n}}$. Since the order of $\operatorname{ker}\left(p_{n}\right)$ divides $a$, the group aut $\left(\operatorname{ker}\left(p_{n}\right)\right)$ contains at most $a$ ! elements, so that any $\phi_{n} \in \operatorname{aut}\left(\operatorname{ker}\left(p_{n}\right)\right)$ satisfies $\phi_{n}^{a !}=\mathrm{id}$. Put $d:=a \cdot a$ !, then we get for every $\phi_{n} \in \operatorname{aut}\left(\operatorname{ker}\left(p_{n}\right)\right)$ and $k \in \operatorname{ker}\left(p_{n}\right)$ the equality

$$
\prod_{i=0}^{d-1} \phi_{n}^{i}(k)=\prod_{j=1}^{a} \prod_{i=(j-1) \cdot a !}^{j \cdot a !-1} \phi_{n}^{i}(k)=\left(\prod_{i=0}^{a !-1} \phi_{n}^{i}(k)\right)^{a}=1 .
$$

Specializing to $\phi_{n}(k):=c_{n}^{r_{n}} k c_{n}^{-r_{n}}$ yields

$$
c_{0}^{d}=\left(k_{n} c_{n}^{r_{n}}\right)^{d}=\left(\prod_{i=0}^{d-1} \phi_{n}^{i}\left(k_{n}\right)\right) \cdot c_{n}^{r_{n} d}=c_{n}^{r_{n} d} .
$$

If $z_{0}$ is a generator of $Z_{0}$, then there exists an $s \in \mathbb{Z}$ such that $p_{0}\left(z_{0}\right)=p_{0}\left(c_{0}\right)^{s}$. This means $z_{0}^{-1} c_{0}^{s} \in \operatorname{ker}\left(p_{0}\right)$, hence $z_{0}^{d}=c_{0}^{s d}$. Altogether this implies that if $d Z_{0}$ denotes the cyclic group generated by $z_{0}^{d}$, we have $d Z_{0}=\left\langle c_{n}^{r_{n}} d s\right\rangle$ and thus $c_{n} \in C_{G} d Z_{0}$.

We can finally define $b:=\left[C_{G} d Z_{0}: d Z_{0}\right]$, which is finite by assumption and constitutes the required constant. This is due to the fact that $c_{n}^{b} \in d Z_{0}$, so there is a $t_{n} \in \mathbb{Z}$ such that $c_{n}^{b}=c_{n}^{r_{n} d s t_{n}}$. Hence $r_{n}$ divides $b$, and the index of $H_{1}\left(C_{G} Z_{0}\right) / T_{0}$ in $H_{1}\left(C_{G} Z_{n}\right) / T_{n}$ equals $\left|r_{n}\right|$ by construction.

Lemma 3.4. Let $G$ be a group with the property that every non-virtually cyclic subgroup of $G$ contains a copy of $\mathbb{Z} * \mathbb{Z}$. Then $G$ satisfies the conditions appearing in Theorem 3.1.

Proof. Let $C \subseteq G$ be infinite cyclic. By assumption, its centralizer $C_{G} C$ is either virtually cyclic or contains $\mathbb{Z} * \mathbb{Z}$. To prove the first condition appearing in Theorem 3.1, we have to show that $C_{G} C$ does not contain $\mathbb{Z} * \mathbb{Z}$. Suppose it does. Then $\mathbb{Z} * \mathbb{Z} \cap C=\{1\}$ as $\mathbb{Z} * \mathbb{Z}$ has a trivial center. Hence one of the generators of $\mathbb{Z} * \mathbb{Z}$ together with a generator of $C$ generate a copy of $\mathbb{Z} \oplus \mathbb{Z}$ inside $G$, which contradicts the assumption imposed on $G$.

It is obvious that any ascending chain $H_{1} \subseteq H_{2} \subseteq \ldots$ of finite subgroups of $G$ must become stationary since, otherwise, $\bigcup_{n} H_{n}$ would be an infinite torsion 
subgroup of $G$, contradicting the assumptions on $G$. This proves the second condition appearing in Theorem 3.1.

Remark 3.5. Lemma 3.4 implies that the main result of Juan-Pineda and Leary in [10] is covered by Theorem 3.1. Obviously this is also true for [16, Theorem 8.11] which motivated Theorem 3.1.

It follows from the Kurosh subgroup theorem (see for example [17, Theorem I.5.14 on page 56]) that the class of groups satisfying the conditions of Theorem 3.1 is closed under arbitrary free products, whereas this is not the case for the class of groups considered in [16, Theorem 8.11].

Example 3.6 (Word-hyperbolic groups). Each word-hyperbolic group $G$ satisfies the two conditions appearing in Theorem 3.1. A proof for this can be found in [3, Theorem 3.2 in III.Г. 3 on page 459 and Corollary 3.10 in III.Г.3 on page 462].

Example 3.7 (A group not satisfying $\left(M_{\mathcal{F} \mathcal{I N} \subseteq \mathcal{V} \mathcal{C Y}}\right)$ ). Let $G$ be the semi-direct product $\mathbb{Z} \rtimes \mathbb{Z}$ with respect to the group homomorphism $\mathbb{Z} \rightarrow$ aut $(\mathbb{Z})$ sending the generator to $-\mathrm{id}: \mathbb{Z} \rightarrow \mathbb{Z}$. A presentation of $G$ is given by $\left\langle s, t \mid s t s^{-1}=t^{-1}\right\rangle$. Consider the infinite cyclic subgroups $\langle s\rangle$ and $\langle s t\rangle$. They are maximal among infinite virtually cyclic subgroups. These two groups are obviously different. Their

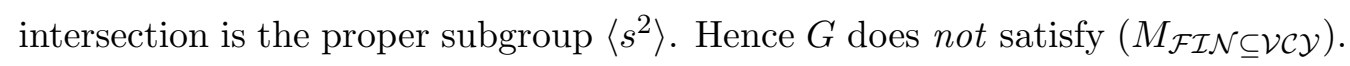

\section{Models for $E_{\mathcal{F}}(G)$ for colimits of groups}

In this section, we want to construct models for $E_{\mathcal{F}}(G)$ if $G$ is a colimit of a directed system of groups $\left\{G_{i} \mid i \in I\right\}$.

Given a directed set $I=(I, \leq)$, we consider $I$ as a category. Namely, the set of objects is the set $I$ itself and $\operatorname{mor}_{I}(i, j)$ consists of precisely one element if $i \leq j$ and is empty otherwise. A directed system of groups is a covariant functor from the category $I$ into the category of groups, i.e., it consists of a collection $\left\{G_{i} \mid i \in I\right\}$ of groups together with group homomorphisms $\phi_{i, j}: G_{i} \rightarrow G_{j}$ for $i \leq$ $j$. Its colimit $G:=\operatorname{colim}_{i \in I} G_{i}$ is a group $G$ together with group homomorphisms $\psi_{i}: G_{i} \rightarrow G$ for every $i \in I$ which satisfy $\psi_{j}=\psi_{i} \circ \phi_{i, j}$ for $i, j \in I$ and $i \leq j$ such that the following universal property holds: Given a group $K$ together with 
group homomorphisms $\mu_{i}: G_{i} \rightarrow K$ satisfying $\mu_{j}=\mu_{i} \circ \phi_{i, j}$ for $i, j \in I$ and $i \leq j$, there is precisely one group homomorphism $\nu: G \rightarrow K$ satisfying $\nu \circ \psi_{i}=\mu_{i}$ for every $i \in I$. If $\left\{G_{i} \mid i \in I\right\}$ is a directed system of subgroups of $G$ directed by inclusion, then $G=\operatorname{colim}_{i \in I} G_{i}$ if and only if $G=\bigcup_{i \in I} G_{i}$.

Given a homomorphism $\psi: G^{\prime} \rightarrow G$ of groups and a family of subgroups $\mathcal{F}$ of $G$, define $\psi^{*} \mathcal{F}$ to be the family of subgroups of $G^{\prime}$ given by $\left\{H \subseteq G^{\prime} \mid \psi(H) \in \mathcal{F}\right\}$. If $\psi$ is an inclusion of groups, then $\psi^{*} \mathcal{F}$ agrees with the family $\mathcal{F} \cap G^{\prime}$ consisting of all the subgroups of $G^{\prime}$ which belong to $\mathcal{F}$.

Definition 4.1 (Homotopy dimension). Given a $G$-space $X$, the homotopy $d i$ mension $\operatorname{hdim}^{G}(X) \in\{0,1, \ldots\} \amalg\{\infty\}$ of $X$ is defined to be the infimum over the dimensions of all $G$-CW-complexes $Y$ which are $G$-homotopy equivalent to $X$.

Obviously, $\operatorname{hdim}^{G}(X)$ depends only on the $G$-homotopy type of $X$. In particular, $\operatorname{hdim}^{G}\left(E_{\mathcal{F}}(G)\right)$ is well-defined for any group $G$ and any family $\mathcal{F}$. For example, $\operatorname{hdim}^{G}\left(E_{\mathcal{F}}(G)\right)=0$ is equivalent to the condition that $\mathcal{F}=\mathcal{A L} \mathcal{L}$.

Let $\mathcal{C}$ be a small category. A contravariant $\mathcal{C}-C W$-complex is a contravariant functor from $\mathcal{C}$ to the category of $C W$-complexes which is built of cells of the shape $\operatorname{mor}_{\mathcal{C}}(?, c) \times D^{n}$. For a rigorous definition of this object, we refer for instance to [6, Definition 3.2] (where it is called a contravariant free $\mathcal{C}-C W$ complex). A model for the contravariant classifying space $E \mathcal{C}$ of the category $\mathcal{C}$ is, by definition, a contravariant $\mathcal{C}-C W$-complex whose evaluation at any object in $\mathcal{C}$ is contractible. Let $\operatorname{hdim}(\mathcal{C})$ be the infimum of the dimensions of all models for $E \mathcal{C}$. The elementary proof of the next result is left to the reader (see $[6$, Example 3.9])).

Lemma 4.2. Let I be a directed set. Then I contains a maximal element if and only if $\operatorname{hdim}(I)=0$. If I contains a countable cofinal subset $I^{\prime}$, then $\operatorname{hdim}(I) \leq 1$. (Cofinal means that for every $i \in I$ there exists $i^{\prime} \in I$ with $i \leq i^{\prime}$.)

Now we can formulate the main result of this section, whose proof is carried out in the remainder of this section.

Theorem 4.3. Let $\left\{G_{i} \mid i \in I\right\}$ be a directed system of groups (with not necessarily injective structure maps $\phi_{i, j}: G_{i} \rightarrow G_{j}$ for $\left.i \leq j\right)$. Let $G=\operatorname{colim}_{i \in I} G_{i}$ be 
the colimit of this directed system. Let $\mathcal{F}$ be a family of subgroups of $G$ such that every $H \in \mathcal{F}$ is contained in $\operatorname{im}\left(\psi_{i}\right)$ for some $i \in I$, where $\psi_{i}: G_{i} \rightarrow G$ is the structure map of the colimit for $i \in I$. Then

$$
\operatorname{hdim}^{G}\left(E_{\mathcal{F}}(G)\right) \leq \operatorname{hdim}(I)+\sup \left\{\operatorname{hdim}^{G_{i}}\left(E_{\psi_{i}^{*} \mathcal{F}}\left(G_{i}\right)\right) \mid i \in I\right\} .
$$

We need some preparations for the proof of Theorem 4.3. In the following, we will use the notation and results of [6, Section 1 and 3], except that we replace the notation $\otimes_{\mathcal{C}}$ in $\left[6\right.$, Definition 1.4] by $\times_{\mathcal{C}}$. We also mention that, for a covariant functor $X: \mathcal{C} \rightarrow$ SPACES, the space $E \mathcal{C} \times_{\mathcal{C}} X$ is a model for the homotopy colimit of $X$ (see [6, page 225]).

Lemma 4.4. Let $\mathcal{C}$ be a small category. Let $Z$ be a contravariant $\mathcal{C}-C W$-complex and let $X: \mathcal{C} \rightarrow G$-SPACES be a covariant functor from $\mathcal{C}$ to the category of $G$ spaces.

Then $Z \times_{\mathcal{C}} X$ is a $G$-space and we have:

(i) If $X(c)$ is a $G$-CW-complex for every $c \in \mathcal{C}$, then $Z \times_{\mathcal{C}} X$ has the homotopy type of a $G$-CW-complex. We have

$$
\operatorname{hdim}^{G}\left(Z \times_{\mathcal{C}} X\right) \leq \operatorname{dim}(Z)+\sup \left\{\operatorname{hdim}^{G}(X(c)) \mid c \in \mathrm{ob}(\mathcal{C})\right\} ;
$$

(ii) For every $H \subseteq G$ there is a natural homeomorphism of spaces

$$
Z \times_{\mathcal{C}} X^{H} \stackrel{\cong}{\longrightarrow}\left(Z \times_{\mathcal{C}} X\right)^{H}
$$

Proof. (i) Let $Z_{n}$ be the $n$-skeleton of $Z_{n}$. We construct, by induction over $n=-1,0,1, \ldots$, a sequence of inclusions of $G$-CW-complexes $Y_{-1} \subseteq Y_{0} \subseteq$ $Y_{1} \subseteq \ldots$ together with $G$-homotopy equivalences $f_{n}: Z_{n} \times_{\mathcal{C}} X \rightarrow Y_{n}$ such that $\left.f_{n}\right|_{Z_{n-1} \times_{\mathcal{C}} X}=f_{n-1}$ and

$$
\operatorname{dim}\left(Y_{n}\right) \leq n+\sup \left\{\operatorname{hdim}^{G}(X(c)) \mid c \in \operatorname{ob}(\mathcal{C})\right\}
$$

holds for $n=0,1,2, \ldots$ Then the claim is obviously true if $Z$ is finite-dimensional. For infinite-dimensional $Z$, a standard colimit argument implies that the system $\left(f_{n}\right)_{n \in \mathbb{N}}$ of $G$-homotopy equivalences yields a $G$-homotopy equivalence 
$f: Z \times_{\mathcal{C}} X \rightarrow Y:=\bigcup_{n \in \mathbb{N}} Y_{n}$ since every map $Z_{n-1} \times_{\mathcal{C}} X \rightarrow Z_{n} \times_{\mathcal{C}} X$ is a $G$ cofibration and $Z \times_{\mathcal{C}} X$ is $\bigcup_{n \in \mathbb{N}} Z_{n} \times_{\mathcal{C}} X_{n}$ with respect to the weak topology. As $Y$ is a $G-C W$-complex, the claim follows in this case as well.

The induction begins by taking $Y_{-1}=\emptyset$. The induction step from $n-1$ to $n \geq 0$ is done as follows. We can write $Z_{n}$ as a pushout of contravariant $\mathcal{C}$-spaces

$$
\begin{gathered}
\bigsqcup_{j \in J} \operatorname{mor}_{\mathcal{C}}\left(?, c_{j}\right) \times S^{n-1} \longrightarrow Z_{n-1} \\
\amalg_{j \in J} \operatorname{id}_{\text {mor }_{\mathcal{C}}\left(?, c_{j}\right)} \times i \mid \\
\coprod_{j \in J} \operatorname{mor}_{\mathcal{C}}\left(?, c_{j}\right) \times D^{n} \longrightarrow Z_{n}^{\perp}
\end{gathered}
$$

where $i: S^{n-1} \rightarrow D^{n}$ is the inclusion. Applying $-\times_{\mathcal{C}} X$ yields a $G$-pushout

$$
\begin{aligned}
& \coprod_{j \in J} X\left(c_{j}\right) \times S^{n-1} \stackrel{u}{\longrightarrow} Z_{n-1} \times_{\mathcal{C}} X
\end{aligned}
$$

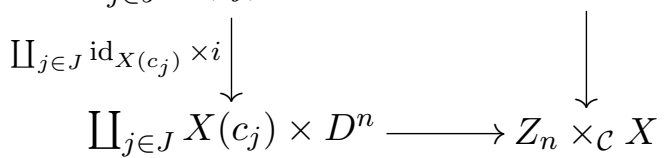

Obviously, the left vertical arrow is a $G$-cofibration and hence the same is true for the right vertical arrow. Choose, for every $c \in \mathcal{C}$, a $G$-CW-complex $X(c)^{\prime}$ of dimension $\operatorname{hdim}^{G}(X(c))$ together with a $G$-homotopy equivalence $u_{c}: X(c)^{\prime} \rightarrow$ $X(c)$. By the equivariant cellular approximation theorem (see for instance $[13$, Theorem 2.1 on page 32$]$ ), the composition

$$
\coprod_{j \in J} X\left(c_{j}\right)^{\prime} \times S^{n-1} \stackrel{\amalg_{j \in J} u_{c_{j}} \times \mathrm{id}_{S^{n-1}}}{\longrightarrow} \coprod_{j \in J} X\left(c_{j}\right) \times S^{n-1} \stackrel{u}{\longrightarrow} Z_{n-1} \times_{\mathcal{C}} X \stackrel{f_{n-1}}{\longrightarrow} Y_{n-1}
$$

is $G$-homotopic to some cellular $G$-map $v: \coprod_{j \in J} X\left(c_{j}\right)^{\prime} \times S^{n-1} \rightarrow Y_{n-1}$. Define $Y_{n}$ by the $G$-pushout

$$
\begin{gathered}
\bigsqcup_{j \in J} X\left(c_{j}\right)^{\prime} \times S^{n-1} \stackrel{v}{\longrightarrow} Y_{n-1} \\
\amalg_{j \in J} \operatorname{id}_{X\left(c_{j}\right)^{\prime}} \times i \mid \\
\amalg_{j \in J} X\left(c_{j}\right)^{\prime} \times D^{n} \longrightarrow Y_{n}
\end{gathered}
$$

This is a $G-C W$-complex whose dimension satisfies

$$
\operatorname{dim}\left(Y_{n}\right) \leq \max \left\{n+\sup \left\{\operatorname{dim}\left(X\left(c_{j}\right)^{\prime}\right) \mid j \in J\right\}, \operatorname{dim}\left(Y_{n-1}\right)\right\},
$$


and the induction hypothesis applied to $Y_{n-1}$ implies

$$
\operatorname{dim}\left(Y_{n}\right) \leq n+\sup \left\{\operatorname{hdim}^{G}(X(c)) \mid c \in \operatorname{ob}(\mathcal{C})\right\} .
$$

Having shown this, we beg the reader's pardon for omitting the actual construction of the $G$-homotopy equivalence $f_{n}: Z_{n} \times_{\mathcal{C}} X \rightarrow Y_{n}$. This construction is based on various standard cofibration arguments and is essentially the same as in the proof that the $G$-homotopy type of a $G$-CW-complex depends only on the $G$-homotopy classes of the attaching maps.

(ii) There is an obvious natural map

$$
f_{Z}: Z \times_{\mathcal{C}} X^{H} \rightarrow\left(Z \times_{\mathcal{C}} X\right)^{H},
$$

and analogously for $Z_{n}$ in place of $Z$. We show by induction over $n$ that the map $f_{Z_{n}}$ is a homeomorphism. Then the claim follows for all finite-dimensional $\mathcal{C}-C W$ complexes $Z$. The infinite-dimensional case follows using a colimit argument.

In the induction step, just note that the various homeomorphisms $f_{Z_{n-1}}$ induce a map from the pushout

$$
\begin{gathered}
\coprod_{j \in J} X\left(c_{j}\right)^{H} \times S^{n-1} \longrightarrow Z_{n-1} \times_{\mathcal{C}} X^{H} \\
\amalg_{j \in J} \operatorname{id}_{X\left(c_{j}\right)^{H}} \times i \downarrow \\
\coprod_{j \in J} X\left(c_{j}\right)^{H} \times D^{n} \longrightarrow Z_{n} \times_{\mathcal{C}} X^{H}
\end{gathered}
$$

to the pushout

$$
\begin{aligned}
\left(\coprod_{j \in J} X\left(c_{j}\right) \times S^{n-1}\right)^{H} & \longrightarrow\left(Z_{n-1} \times_{\mathcal{C}} X\right)^{H} \\
\left(\amalg_{j \in J} \operatorname{id}_{X\left(c_{j}\right)} \times i\right)^{H} \downarrow & \downarrow \\
\left(\coprod_{j \in J} X\left(c_{j}\right) \times D^{n}\right)^{H} & \longrightarrow\left(Z_{n} \times_{\mathcal{C}} X\right)^{H}
\end{aligned}
$$

and that a pushout of homeomorphisms is again a homeomorphism.

Lemma 4.5. Let $\left\{G_{i} \mid i \in I\right\}$ be a directed system of groups. Let $G=\operatorname{colim}_{i \in I} G_{i}$ be its colimit. Let $\mathcal{F}$ be a family of subgroups of $G$ such that every $H \in \mathcal{F}$ is contained in $\operatorname{im}\left(\psi_{i}\right)$ for some $i \in I$. We obtain a covariant functor $G / \operatorname{im}\left(\psi_{\text {? }}\right): I \rightarrow$ $G$-SPACES by sending $i$ to $G / \operatorname{im}\left(\psi_{i}\right)$.

Then $E I \times_{I} G / \operatorname{im}\left(\psi_{\text {? }}\right)$ is $G$-homotopy equivalent to $\{\bullet\}$. 
Proof. We already know from Lemma 4.4 (i) that $E I \times_{I} G / \operatorname{im}\left(\psi_{\text {? }}\right)$ has the $G$ homotopy type of a $G$-CW-complex (one can actually check easily that it is itself a $G$ - $C W$-complex, the $n$-skeleton being $\left.E I_{n} \times{ }_{I} G / \operatorname{im}\left(\psi_{\text {? }}\right)\right)$. Hence, by the equivariant Whitehead theorem (see for instance [13, Theorem 2.4 on page 36]), it suffices to show that $\left(E I \times{ }_{I} G / \operatorname{im}\left(\psi_{\text {? }}\right)\right)^{H}$ is weakly contractible for every subgroup $H \subseteq G$. Because of Lemma 4.4 (ii), it remains to show for every $H \subseteq G$ that $E I \times_{I}\left(G / \operatorname{im}\left(\psi_{\text {? }}\right)\right)^{H}$ is weakly contractible.

Recall that $E I \times_{I}\left(G / \operatorname{im}\left(\psi_{\text {? }}\right)\right)^{H}$ is defined as $\coprod_{i \in I} E I(i) \times\left(G / \operatorname{im}\left(\psi_{i}\right)\right)^{H} / \sim$ for some tensor product-like equivalence relation $\sim($ see $[6$, Definition 1.4] $)$. Consider two points $x_{1}$ and $x_{2}$ in $E I \times_{I}\left(G / \operatorname{im}\left(\psi_{?}\right)\right)^{H}$. Since $G=\bigcup_{i \in I} \operatorname{im}\left(\psi_{i}\right)$ and, by assumption, $I$ is directed and there is an index $j \in I$ with $H \subseteq \operatorname{im}\left(\psi_{j}\right)$, we can find an index $k \in I$ and $z_{1}, z_{2} \in E I(k)$ such that $\left(z_{l}, 1 \cdot \operatorname{im}\left(\psi_{k}\right)\right) \in E I(k) \times G / \operatorname{im}\left(\psi_{k}\right)$ lies in $E I(k) \times\left(G / \operatorname{im}\left(\psi_{k}\right)\right)^{H}$ and represent $x_{l}$ for $l=1,2$. As $E I(k)$ is contractible and in particular path-connected, we can join $z_{1}$ and $z_{2}$ and hence $x_{1}$ and $x_{2}$ by a path. This shows that $E I \times_{I}\left(G / \operatorname{im}\left(\psi_{\text {? }}\right)\right)^{H}$ is path-connected.

It remains to show for every compact subspace $C \subseteq E I \times_{I}\left(G / \operatorname{im}\left(\psi_{\text {? }}\right)\right)^{H}$ that we can find a subspace $D \subseteq E I \times_{I}\left(G / \operatorname{im}\left(\psi_{\text {? }}\right)\right)^{H}$ such that $C \subseteq D$ and such that $D$ is homotopy equivalent to a discrete set. In the following, we use the functorial bar model as described in [6, page 230] as the model for EI. Notice that the $n$-cells in $E I$ are indexed by the set of all sequences $i_{0}<i_{1}<\ldots<i_{n}$ of elements in $I$. Since $C$ is compact, it is contained in the union of finitely many of the equivariant cells of $E I \times{ }_{I} G / \operatorname{im}\left(\psi_{\text {? }}\right)$. Such an equivariant cell is of the shape $\left(\operatorname{mor}_{I}(?, i) \times D^{n}\right) \times{ }_{I} G / \operatorname{im}\left(\psi_{?}\right)=G / \operatorname{im}\left(\psi_{i}\right) \times D^{n}$ for some cell $\operatorname{mor}_{I}(?, i) \times D^{n}$ of $E I$. Since $I$ is directed, we can find an index $j \in I$ such that $C$ is already contained in $(E I)_{\leq j} \times_{I} G / \operatorname{im}\left(\psi_{?}\right)$, where $(E I)_{\leq j}$ is the $I$ - $C W$-subcomplex of $E I$ built of cells for which the indexing sequence $i_{0}<i_{1}<\ldots<i_{n}$ satisfies $i_{n} \leq j$. Let $k_{j}: I_{\leq j} \rightarrow I$ be the inclusion of the full subcategory $I_{\leq j}$ of $I$ formed by the objects $i \in I$ with $i \leq j$. Then $(E I)_{\leq j}$ is isomorphic to the induction $\left(k_{j}\right)_{*} E\left(I_{\leq j}\right)$ of the bar model $E\left(I_{\leq j}\right)$ of the classifying space for the category $I_{\leq j}$. However, $I_{\leq j}$ has a terminal object, namely $j$, so the canonical projection $E\left(I_{\leq j}\right) \rightarrow \operatorname{mor}_{I_{\leq j}}(?, j)$ is a homotopy equivalence of contravariant $I_{\leq j}$-spaces. It 
induces a homotopy equivalence

$$
\begin{aligned}
(E I)_{\leq j} \times_{I} G / \operatorname{im}\left(\psi_{?}\right) \cong\left(k_{j}\right)_{*} E\left(I_{\leq j}\right) \times_{I} G / \operatorname{im}\left(\psi_{?}\right) \\
\\
\stackrel{\simeq}{\cong}\left(k_{j}\right)_{*} \operatorname{mor}_{I_{\leq j}}(?, j) \times_{I} G / \operatorname{im}\left(\psi_{?}\right)=G / \operatorname{im}\left(\psi_{j}\right) .
\end{aligned}
$$

Summarizing, $C \subseteq D$ holds for $D:=(E I)_{\leq j} \times_{I} G / \operatorname{im}\left(\psi_{\text {? }}\right)$, and $D$ is homotopy equivalent to a discrete set.

Now we can give the proof of Theorem 4.3.

Proof. Fix a model $E_{\mathcal{F}}(G)$. We get a covariant functor $G / \operatorname{im}\left(\psi_{\text {? }}\right) \times E_{\mathcal{F}}(G): I \rightarrow$ $G$-SPACES by sending $i$ to $G / \operatorname{im}\left(\psi_{i}\right) \times E_{\mathcal{F}}(G)$ and, for $i \leq j$, by sending the morphism $i \rightarrow j$ in $I$ to the $G$-map pr $\times$ id: $G / \operatorname{im}\left(\psi_{i}\right) \times E_{\mathcal{F}}(G) \rightarrow G / \operatorname{im}\left(\psi_{j}\right) \times$ $E_{\mathcal{F}}(G)$, where pr is the obvious projection. From Lemma 4.5 , we obtain a $G$ homotopy equivalence

$$
\begin{aligned}
E I \times_{I}\left(G / \operatorname{im}\left(\psi_{?}\right) \times E_{\mathcal{F}}(G)\right) \cong\left(E I \times_{I} G / \operatorname{im}\left(\psi_{?}\right)\right) & \times E_{\mathcal{F}}(G) \\
& \stackrel{\simeq}{\longrightarrow}\{\bullet\} \times E_{\mathcal{F}}(G)=E_{\mathcal{F}}(G) .
\end{aligned}
$$

The $G$-space $G / \operatorname{im}\left(\psi_{i}\right) \times E_{\mathcal{F}}(G)$ is a $G$-CW-complex for every $i$ and is $G$ homeomorphic to $G \times_{G_{i}} \psi_{i}^{*} E_{\mathcal{F}}(G)$, where $\psi_{i}^{*} E_{\mathcal{F}}(G)$ is the $G_{i^{-}} C W$-complex obtained from the $G$-CW-complex $E_{\mathcal{F}}(G)$ by restriction with $\psi_{i}$. One easily checks that $\psi_{i}^{*} E_{\mathcal{F}}(G)$ is a model for $E_{\psi_{i}^{*} \mathcal{F}}\left(G_{i}\right)$. Hence, the $G$-space $G / \operatorname{im}\left(\psi_{i}\right) \times E_{\mathcal{F}}(G)$ is $G$-homotopy equivalent to a $G$-CW-complex of dimension $\operatorname{hdim}^{G_{i}}\left(E_{\psi_{i}^{*} \mathcal{F}}\left(G_{i}\right)\right)$. Now the claim follows from Lemma 4.4 (i) applied to $Z=E I$ and $X=G / \operatorname{im}\left(\psi_{\text {? }}\right) \times$ $E_{\mathcal{F}}(G)$.

\section{On the dimension of $\underline{\underline{E}} G$}

In this section, we deal with the question what can be said about the dimension of $G-C W$-complexes which are models for $\underline{\underline{E}} G$. The same question for $\underline{E} G$ has already been thoroughly investigated, and in many cases satisfying answers are known (see for instance [16], where further references to relevant papers are given). 


\subsection{Lower bounds for the homotopy dimension for $\mathcal{V C Y}$ by the one for $\mathcal{F I N}$}

In this subsection, we give lower bounds for $\operatorname{hdim}^{G}(\underline{\underline{E}} G)$ in terms of $\operatorname{hdim}^{G}(\underline{E} G)$. We have introduced the notion of the homotopy dimension $\operatorname{hdim}^{G}(X)$ of a $G$ $C W$-complex $X$ in Definition 4.1.

Proposition 5.1. Let $\mathcal{F} \subseteq \mathcal{G}$ be families of subgroups of $G$.

(i) Let $n \geq 0$ be an integer. Suppose that for any $H \in \mathcal{G}$ there is an $n$ dimensional model for $E_{\mathcal{F} \cap H}(H)$. Then

$$
\operatorname{hdim}^{G}\left(E_{\mathcal{F}}(G)\right) \leq n+\operatorname{hdim}^{G}\left(E_{\mathcal{G}}(G)\right) ;
$$

(ii) Suppose that there exists a finite $H-C W$-model for $E_{\mathcal{F} \cap H}(H)$ for every $H \in \mathcal{G}$ and a finite $G$-CW-model for $E_{\mathcal{G}}(G)$. Then there exists a finite $G$-CW-model for $E_{\mathcal{F}}(G)$.

The same is true if we replace "finite" by "of finite type" everywhere.

Proof. We only give the proof of assertion (i) since the one for assertion (ii) is similar.

Let $Z$ be an $m$-dimensional $G$-CW-complex with isotropy groups in $\mathcal{G}$. We will show that then $Z \times E_{\mathcal{F}}(G)$ is $G$-homotopy equivalent to an $(n+m)$-dimensional $G$-CW-complex, which implies the claim of the proposition as $E_{\mathcal{G}}(G) \times E_{\mathcal{F}}(G)$ is a model for $E_{\mathcal{F}}(G)$.

We utilize induction over the dimension $d$ of $Z$. If $Z=\emptyset$, then there is nothing to show, so let $d \geq 0$. Crossing the $G$-pushout telling how $Z_{d}$ arises from $Z_{d-1}$ with $E_{\mathcal{F}}(G)$ yields a $G$-pushout

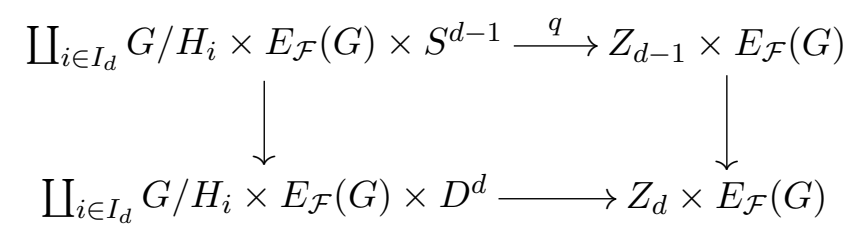

There is a $G$-homotopy equivalence $f_{i}: G \times_{H_{i}} E_{\mathcal{F} \cap H_{i}}\left(H_{i}\right) \rightarrow G / H_{i} \times E_{\mathcal{F}}(G)$ since the restriction of $E_{\mathcal{F}}(G)$ to $H_{i}$ is a $H_{i}$ - $C W$-model for $E_{\mathcal{F} \cap H_{i}}\left(H_{i}\right)$. We 
put $f_{S}:=\coprod_{i} f_{i} \times \operatorname{id}_{S^{d-1}}$ and $f_{D}:=\coprod_{i} f_{i} \times \operatorname{id}_{D^{d}}$. Furthermore, by induction hypothesis, there is a $G$-homotopy equivalence $h: Z^{\prime} \rightarrow Z_{d-1} \times E_{\mathcal{F}}(G)$, where $Z^{\prime}$ is an $(n+d-1)$-dimensional $G$ - $C W$-complex. Choose a $G$-homotopy inverse $k$ of $h$.

For $j: \coprod_{i} G \times_{H_{i}} E_{\mathcal{F} \cap H_{i}}\left(H_{i}\right) \times S^{d-1} \rightarrow \operatorname{cyl}\left(k \circ q \circ f_{S}\right)$ and $p: \operatorname{cyl}\left(k \circ q \circ f_{S}\right) \rightarrow Z^{\prime}$ the obvious inclusion and projection, $h \circ p \circ j$ and $q \circ f_{S}$ are $G$-homotopy equivalent. Since $j$ is a $G$-cofibration, $h \circ p$ can be altered within its $G$-homotopy class to yield a $G$-map $h^{\prime}: \operatorname{cyl}\left(k \circ q \circ f_{S}\right) \rightarrow Z_{d-1} \times E_{\mathcal{F}}(G)$ such that $h^{\prime} \circ j=q \circ f_{S}$. Now consider the $(n+d)$-dimensional $G$ - $C W$-complex $Z^{\prime \prime}$ which is defined by the $G$-pushout

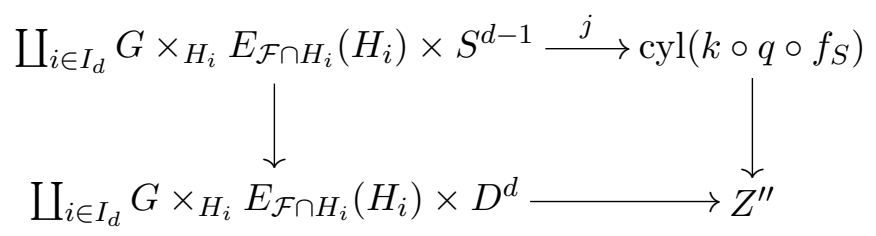

The $G$-homotopy equivalences $f_{S}, f_{D}$ and $h^{\prime}$ induce a map of $G$-pushouts from (5.3) to (5.2), and, as the left vertical arrows in these diagrams are $G$-cofibrations, $Z_{d} \times E_{\mathcal{F}}(G)$ is $G$-homotopy equivalent to $Z^{\prime \prime}$ by [13, Lemma 2.13].

Since any virtually cyclic group $V$ admits a finite 1-dimensional model for $\underline{E} V$, Proposition 5.1 implies:

Corollary 5.4. Let $G$ be a group.

(i) We have $\operatorname{hdim}^{G}(\underline{E} G) \leq 1+\operatorname{hdim}^{G}(\underline{\underline{E}} G)$;

(ii) If there is a model for $\underline{\underline{E}} G$ which is finite or of finite type, then there is a model for $\underline{E} G$ which is finite or of finite type respectively.

Remark 5.5. The inequality appearing in Corollary 5.4 (i) is sharp (see Example 5.29, Theorem 5.33 (i) and Example 5.34).

Corollary 5.6. If $G$ and $H$ are groups, then

$$
\operatorname{hdim}^{G \times H}(\underline{\underline{E}}(G \times H)) \leq \operatorname{hdim}^{G}(\underline{\underline{E}} G)+\operatorname{hdim}^{H}(\underline{\underline{E}} H)+3 .
$$


Proof. Let $\mathcal{F}$ be the family of subgroups of $G \times H$ which are contained in subgroups of the shape $V \times W$ for virtually cyclic subgroups $V \subseteq G$ and $W \subseteq H$. Then $\underline{\underline{E}} G \times \underline{\underline{E}} H$ is a model for $E_{\mathcal{F}}(G \times H)$. An element $K$ in $\mathcal{F}$ contains $\mathbb{Z}^{n}$ for some $n \in\{0,1,2\}$ as subgroup of finite index. This implies $\operatorname{hdim}^{K}(\underline{\underline{E}} K) \leq 3$ (see Example 5.21). Now apply Proposition 5.1 to $G \times H$ for $\mathcal{V C Y} \subseteq \mathcal{F}$.

Remark 5.7. The inequality appearing in Corollary 5.6 is sharp, as can already be seen in the case $G=H=\mathbb{Z}$ (see Example 5.21).

For another example, let $G_{-1}$ be the group appearing in Example 5.29. Then $\operatorname{hdim}^{G_{-1}}\left(\underline{\underline{E}} G_{-1}\right)=3$. Since the center of $G_{-1} \times G_{-1}$ is isomorphic to $\mathbb{Z}^{2}$ and $\operatorname{vcd}\left(G_{-1} \times G_{-1}\right)=\operatorname{vcd}\left(G_{-1}\right)+\operatorname{vcd}\left(G_{-1}\right)=8$ by Lemma 5.14 (iv), Theorem 5.13 (iii) implies $\operatorname{hdim}^{G_{-1} \times G_{1}}\left(\underline{\underline{E}}\left(G_{-1} \times G_{-1}\right)\right)=9$.

\subsection{Upper bounds for the homotopy dimension for $\mathcal{V} \mathcal{Y} \mathcal{Y}$ by the one for $\mathcal{F I N}$}

The goal of this subsection is to give upper bounds for $\operatorname{hdim}^{G}(\underline{\underline{E}} G)$ in terms of $\operatorname{hdim}^{G}(\underline{E} G)$. Recall Notation 2.7.

Theorem 5.8. (i) Let $G$ be a group satisfying $M_{\mathcal{F} \mathcal{I N} \subseteq \mathcal{V} \mathcal{Y Y}}$. Suppose that one of the following conditions is satisfied:

(a) For every maximal infinite virtually cyclic subgroup $V \subseteq G$ of type $I$ we have

$$
\operatorname{dim}\left(E W_{G} V\right)<\infty
$$

(b) We have: $\operatorname{hdim}^{G}(\underline{\underline{E}} G)<\infty$.

Then:

$$
\operatorname{hdim}^{G}(\underline{\underline{E}} G) \leq \operatorname{hdim}^{G}(\underline{E} G)+1
$$

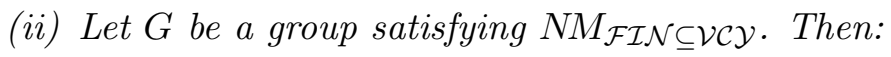

$$
\operatorname{hdim}^{G}(\underline{\underline{E}} G) \begin{cases}=\operatorname{hdim}^{G}(\underline{E} G) & \text { if } \operatorname{hdim}^{G}(\underline{E} G) \geq 2 \\ \leq 2 & \text { if } \operatorname{hdim}^{G}(\underline{E G}) \leq 1\end{cases}
$$


Proof. (i) We first explain that condition (i)(b) implies condition (i)(a). Choose a finite-dimensional $G$-CW-model for $\underline{\underline{E}} G$. Let $V \subseteq G$ be a maximal infinite virtually cyclic subgroup. The $G$-action on $\underline{\underline{E}} G$ induces a $N_{G} V$-action by restriction and hence a $W_{G} V$-action on $\underline{\underline{E}} G^{V}$. Obviously $\underline{\underline{E}} G^{V}$ is a $W_{G} V$-CW-complex which has finite dimension and is contractible (after forgetting the group action). It suffices to show that it is a model for $E W_{G} V$, i.e., that $W_{G} V$ acts freely on $\underline{\underline{E}} G^{V}$. For $x \in \underline{\underline{E}} G^{V}$ the isotropy group $G_{x}$ under the $G$-action is a virtually cyclic subgroup of $G$ which contains $V$. By the maximality of $V$ we conclude $G_{x}=V$. Hence the isotropy group of $x$ under the $W_{G} V$-action is trivial.

Hence it suffices to prove assertion (i) provided that condition (i)(a) is true.

Let cyl $\left(f_{V}\right)$ be the mapping cylinder of the $N_{G} V$-map $f_{V}: \underline{E} N_{G} V \rightarrow E W_{G} V$ appearing in Corollary 2.10. Then we obtain from Corollary 2.10 a cellular $G$ pushout

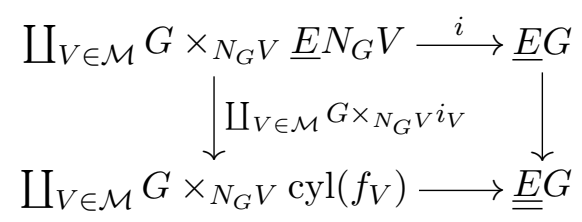

where the $i_{V}$ are the obvious inclusions. Since $\operatorname{res}_{G}^{N_{G} V} \underline{E} G$ is an $N_{G} V-C W$-model for $\underline{E} N_{G} V$, it suffices to show

$$
\operatorname{hdim}^{W_{G} V}\left(E W_{G} V\right) \leq \operatorname{hdim}^{N_{G} V}\left(\underline{E} N_{G} V\right) .
$$

Let $d=\operatorname{hdim}^{W_{G} V}\left(E W_{G} V\right)$ if $\operatorname{hdim}^{W_{G} V}\left(E W_{G} V\right)$ is finite, or let $d$ be any positive integer if $\operatorname{hdim}^{W_{G} V}\left(E W_{G} V\right)$ is infinite. We can choose a left $\mathbb{Z}\left[W_{G} V\right]$-module $M$ such that $H_{W_{G} V}^{d}\left(E W_{G} V ; M\right) \neq 0$ (see [4, Proposition 2.2 on page 185]). Since $W_{G} V$ is torsionfree, $V \backslash \underline{E} N_{G} V$ is a free $W_{G} V$-CW-complex. By the equivariant Whitehead theorem (see [13, Theorem 2.4 on page 36]), the projection $V \backslash \underline{E} N_{G} V \times E W_{G} V \rightarrow V \backslash \underline{E} N_{G} V$ is a $W_{G} V$-homotopy equivalence, where $W_{G} V$ acts diagonally on the source. Since $E W_{G} V$ is a free $W_{G} V-C W$-complex, we obtain a cohomology spectral sequence converging to $H_{W_{G} V}^{p+q}\left(V \backslash \underline{E} N_{G} V ; M\right)$ whose $E_{2}$-term is given by

$$
E_{2}^{p, q}=H_{W_{G} V}^{p}\left(E W_{G} V ; H_{W_{G} V}^{q}\left(V \backslash \underline{E} N_{G} V \times W_{G} V ; M\right)\right) .
$$

Here, $W_{G} V$ acts diagonally from the left on $V \backslash \underline{E} N_{G} V \times W_{G} V$, and the $W_{G} V$ action on $\left.H_{W_{G} V}^{q}\left(V \backslash \underline{E} N_{G} V \times W_{G} V ; M\right)\right)$ comes from the right $W_{G} V$-action on 
$V \backslash \underline{E} N_{G} V \times W_{G} V$ which is given by $\left(x, w^{\prime}\right) \cdot w=\left(x, w^{\prime} w\right)$ (and which commutes with the left $W_{G} V$-action). Now consider the homeomorphism

$$
\phi: V \backslash \underline{E} N_{G} V \times W_{G} V \rightarrow V \backslash \underline{E} N_{G} V \times W_{G} V, \quad(x, w) \mapsto(w x, w) .
$$

It is equivariant with respect to the left $W_{G} V$-actions given by $w \cdot\left(x, w^{\prime}\right)=$ $\left(x, w w^{\prime}\right)$ on the source and the diagonal action on the target. Moreover, it is equivariant with respect to the right $W_{G} V$-actions given by $\left(x, w^{\prime}\right) \cdot w=\left(w^{-1} x, w^{\prime} w\right)$ on the source and by $\left(x, w^{\prime}\right) \cdot w=\left(x, w^{\prime} w\right)$ on the target. Thus, we obtain an isomorphism of abelian groups

$$
\begin{array}{r}
\alpha: H_{W_{G} V}^{q}\left(V \backslash \underline{E} N_{G} V \times W_{G} V ; M\right) \stackrel{H_{W_{G} V}^{q}(\phi ; M)}{\longrightarrow} H_{W_{G} V}^{q}\left(V \backslash \underline{E} N_{G} V \times W_{G} V ; M\right) \\
\stackrel{\mu}{\longrightarrow} H^{q}\left(V \backslash \underline{E} N_{G} V ; M\right) .
\end{array}
$$

where $\mu$ is induced by the isomorphism of $\mathbb{Z}$-cochain complexes

$$
\begin{aligned}
& \operatorname{hom}_{\mathbb{Z}\left[W_{G} V\right]}\left(C_{*}\left(V \backslash \underline{E} N_{G} V \times W_{G} V\right), M\right) \\
& \cong \operatorname{hom}_{\mathbb{Z}\left[W_{G} V\right]}\left(C_{*}\left(V \backslash \underline{E} N_{G} V\right) \otimes_{\mathbb{Z}} \mathbb{Z}\left[W_{G} V\right], M\right) \cong \operatorname{hom}_{\mathbb{Z}}\left(C_{*}\left(V \backslash \underline{E} N_{G} V\right) ; M\right) .
\end{aligned}
$$

The isomorphism $\alpha$ becomes an isomorphism of left $\mathbb{Z}\left[W_{G} V\right]$-modules if we equip the target with the $W_{G} V$-action that comes from the following $W_{G} V$-action on the $\mathbb{Z}$-chain complex $\operatorname{hom}_{\mathbb{Z}}\left(C_{*}\left(V \backslash \underline{E} N_{G} V\right) ; M\right)$ : namely, $w \in W_{G} V$ acts by sending $f$ to $l_{w} \circ f \circ C_{*}\left(l_{w^{-1}}\right)$, where $l_{w}$ and $l_{w^{-1}}$ denotes left multiplication with $w$ and $w^{-1}$. The universal coefficient theorem for the ring $\mathbb{Z}$ yields an exact sequence of $\mathbb{Z}\left[W_{G} V\right]$-modules

$$
\begin{aligned}
& 0 \rightarrow \operatorname{Ext}_{\mathbb{Z}}^{1}\left(H_{q-1}\left(V \backslash \underline{E} N_{G} V\right), M\right) \rightarrow H^{q}\left(V \backslash \underline{E} N_{G} V ; M\right) \\
& \rightarrow \operatorname{hom}_{\mathbb{Z}}\left(H_{q}\left(V \backslash \underline{E} N_{G} V\right), M\right) \rightarrow 0,
\end{aligned}
$$

where the $W_{G} V$-actions on the first and third term are given by combining the obvious right $W_{G} V$-action on $H_{*}\left(V \backslash \underline{E} N_{G} V\right)$ and the given left $W_{G} V$-action on $M$.

Now we separately treat the two cases that $V$ is a virtually cyclic group of type I or of type II. In the first case, we can find an epimorphism $\alpha: V \rightarrow \mathbb{Z}$ with finite kernel. Then $E \mathbb{Z}$, viewed as a $V$-space by restricting with $\alpha$, is a 
model for $\underline{E} V$. Similarly, $\operatorname{res}_{N_{G} V} \underline{E} N_{G} V$ is a model for $\underline{E} V$. Hence, there is a $V$-homotopy equivalence $\underline{E} N_{G} V \rightarrow E \mathbb{Z}$, which induces a homotopy equivalence $V \backslash \underline{E} N_{G} V \rightarrow \mathbb{Z} \backslash E \mathbb{Z}$. We conclude that $H_{q}\left(V \backslash \underline{E} N_{G} V\right)$ is infinite cyclic for $q=0,1$ and trivial for $q \geq 2$. This implies that we obtain an isomorphism of $\mathbb{Z}\left[W_{G} V\right]$ modules

$$
H^{q}\left(V \backslash \underline{E} N_{G} V ; M\right) \stackrel{\cong}{\longrightarrow} \operatorname{hom}_{\mathbb{Z}}\left(H_{q}\left(V \backslash \underline{E} N_{G} V\right), M\right) .
$$

Let $\epsilon: W_{G} V \rightarrow\{ \pm 1\}$ the homomorphism which is uniquely determined by the property that $w \in W_{G} V$ acts on $H_{1}\left(V \backslash \underline{E} N_{G} V\right)$ by multiplication with $\epsilon(w)$. Let $M^{\epsilon}$ be the $\mathbb{Z}\left[W_{G} V\right]$-module whose underlying abelian group is $M$ but on which the $W_{G} V$-action is twisted by $\epsilon$, that is, $w \cdot x$ is defined to be $\epsilon(w) w x$ for $w \in W_{G} V$ and $x \in M$. Then $\operatorname{hom}_{\mathbb{Z}}\left(H_{1}\left(V \backslash \underline{E} N_{G} V\right), M\right)$ is $\mathbb{Z}\left[W_{G} V\right]$-isomorphic to $M^{\epsilon}$. Summarizing, what we have shown so far is that $E_{2}^{p, q}=H_{W_{G} V}^{p}\left(E W_{G} V ; M^{\epsilon}\right)$ for $q=1$ and $E_{2}^{p, q}=0$ for $q \geq 2$. We get $d<\infty$ from condition (i)(a). Obviously $E_{2}^{p, q}=0$ holds for $p>d$. So the $E^{2}$-term of the first quadrant spectral sequence looks like

\begin{tabular}{|c|c|c|c|c|c|}
\hline$\vdots$ & $\vdots$ & $\vdots$ & $\vdots$ & $\vdots$ & $\vdots$ \\
\hline 0 & $\ldots$ & 0 & 0 & 0 & 0 \\
\hline 0 & $\ldots$ & 0 & 0 & 0 & 0 \\
\hline$E_{2}^{0,1}$ & $\cdots$ & $E_{2}^{2, d-1}$ & $H_{W_{G} V}^{d}\left(E W_{G} V ; M^{\epsilon}\right)$ & 0 & 0 \\
\hline$E_{2}^{0,0}$ & . & $E_{2}^{d-1,0}$ & $E_{2}^{d, 0}$ & 0 & 0 \\
\hline
\end{tabular}

Hence we obtain an isomorphism

$$
H_{W_{G} V}^{d+1}\left(V \backslash \underline{E} N_{G} V ; M\right) \cong H_{W_{G} V}^{d}\left(E W_{G} V ; M^{\epsilon}\right) .
$$

Since $\left(M^{\epsilon}\right)^{\epsilon}=M$ and $H_{W_{G} V}^{d}\left(E W_{G} V ; M\right) \neq 0$, this implies that we have $H_{W_{G} V}^{d+1}\left(V \backslash \underline{E} N_{G} V ; M^{\epsilon}\right) \neq 0$. It follows that $\operatorname{hdim}^{N_{G} V}\left(\underline{E} N_{G} V\right) \geq d+1$, and hence assertion (5.9) is true in the case that $V$ is of type I. 
It remains to treat the (easier) case II, in which there is an epimorphism $\alpha: V \rightarrow D_{\infty}$ onto the infinite dihedral group. Just as above, we obtain a homotopy equivalence $V \backslash \underline{E} N_{G} V \rightarrow D_{\infty} \backslash \underline{E} D_{\infty}$, and as $D_{\infty} \backslash \underline{E} D_{\infty}$ is contractible, $H_{q}\left(V \backslash \underline{E} N_{G} V\right)$ is trivial for $q \geq 1$ and is isomorphic as a $\mathbb{Z}\left[W_{G} V\right]$-module to $\mathbb{Z}$ with the trivial $W_{G} V$-action for $q=0$. Hence, the cohomology spectral sequence yields an isomorphism

$$
H_{W_{G} V}^{d}\left(V \backslash \underline{E} N_{G} V ; M\right) \cong H_{W_{G} V}^{d}\left(E W_{G} V ; M\right),
$$

whence it follows that $H_{W_{G} V}^{d}\left(V \backslash \underline{E} N_{G} V ; M\right) \neq 0$. So hdim ${ }^{N_{G} V}\left(\underline{E} N_{G} V\right) \geq d$, and we get assertion (5.9).

(ii) From Corollary 2.11 we get a cellular $G$-pushout

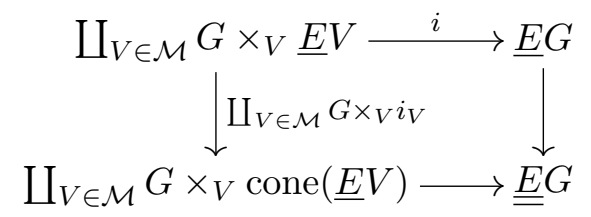

where $\mathcal{M}$ is a complete system of representatives of the conjugacy classes of maximal infinite virtually cyclic subgroups and $i_{V}$ is the obvious inclusion. Since each $V$ is virtually cyclic, we can choose a 1-dimensional model for $\underline{E V}$. Then $\operatorname{cone}(\underline{E} V)$ is 2-dimensional. This implies that there exists a $\max \{d, 2\}$-model for $\underline{\underline{E}} G$ if there is a $d$-dimensional model for $\underline{E} G$. Hence it remains to prove $\operatorname{hdim}^{G}(\underline{E} G) \leq \operatorname{hdim}^{G}(\underline{\underline{E}} G)$ provided that $\operatorname{hdim}^{G}(\underline{E} G) \geq 2$ holds.

Put $d=\operatorname{hdim}^{G}(\underline{E} G)$. We get from [13, Proposition 11.10 on page 221] a contravariant $\mathbb{Z} \operatorname{Or}_{\mathcal{F} \mathcal{I N}}(G)$-module $M$ with $H^{d}\left(\operatorname{hom}_{\mathbb{Z O O}} \operatorname{rof}_{\mathcal{F}(G)}\left(C_{*}(\underline{E} G), M\right)\right) \neq 0$. Define a contravariant $\mathbb{Z O r}_{\mathcal{V C Y}}(G)$-module $\bar{M}$ by putting $\bar{M}(G / H)=M(G / H)$ if $H \in \mathcal{F} \mathcal{I N}$ and $\bar{M}(G / H)=0$ if $H \notin \mathcal{F} \mathcal{I N}$. If $f: G / H \rightarrow G / K$ is a morphism in $\operatorname{Or}_{\mathcal{V C Y}}(G)$, define $\bar{M}(f)=M(f)$ if $H, K \in \mathcal{F} \mathcal{I N}$, and $\bar{M}(f)=0$ otherwise. If $j: \operatorname{Or}_{\mathcal{F} \mathcal{I N}}(G) \rightarrow \operatorname{Or}_{\mathcal{V C Y}}(G)$ is the inclusion, then the restriction $j^{*} \bar{M}$ agrees with $M$. The adjunction between induction and restriction yields an isomorphism of $\mathbb{Z}$-chain complexes

$$
\begin{aligned}
& \left.\left.\operatorname{hom}_{\mathbb{Z O r} r_{\mathcal{F N}}(G)}\left(C_{*}(\underline{E} G), M\right)\right) \cong \operatorname{hom}_{\mathbb{Z O r _ { \mathcal { F I N } }}(G)}\left(C_{*}(\underline{E} G), j^{*} \bar{M}\right)\right) \\
& \left.\cong \operatorname{hom}_{\mathbb{Z} \operatorname{Or}_{\mathcal{V} \mathcal{Y}}(G)}\left(j_{*} C_{*}(\underline{E} G), \bar{M}\right)\right) .
\end{aligned}
$$


Since there exists a 1-dimensional model for $\underline{E} V$ if $V \in \mathcal{M}$ and since $d \geq 2$, we obtain an epimorphism

$$
H^{d}\left(\operatorname{hom}_{\mathbb{Z} \operatorname{Or}_{\mathcal{V} \mathcal{Y}}(G)}\left(C_{*}(\underline{\underline{E}} G), \bar{M}\right)\right) \rightarrow H^{d}\left(\operatorname{hom}_{\mathbb{Z} \operatorname{Or}_{\mathcal{V C Y}}(G)}\left(j_{*} C_{*}(\underline{E} G), \bar{M}\right)\right)
$$

from the long exact cohomology sequence of the pair $(\underline{\underline{E}} G, \underline{E} G)$ with coeffi-

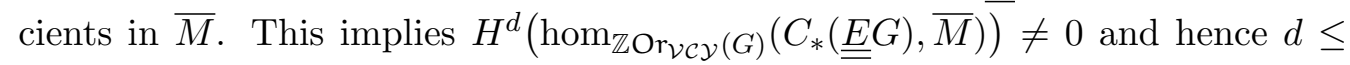
$\operatorname{hdim}^{G}(\underline{\underline{E}} G)$. This finishes the proof of Theorem 5.8.

Remark 5.10. Condition (i)(a) appearing Theorem 5.8 could be avoided if one could show the following: If $1 \rightarrow C \rightarrow \Gamma \rightarrow Q \rightarrow 1$ is an extension of groups such that $C$ is infinite cyclic, $Q$ is torsionfree and there exists a finite-dimensional model for $B \Gamma$, then there exists a finite-dimensional model for $B Q$. However, it is unlikely that such a statement is true, although we have no explicit counterexample.

Suppose that we have such an extension for which no finite-dimensional model for $B Q$ exists. Then the Gysin sequence, whose existence follows from spectral sequence argument appearing in Theorem 5.8, implies that for every $\mathbb{Z} Q$-module $M$ and $n \geq \operatorname{dim}(B \Gamma)$ we have $H_{Q}^{n}(E Q ; M) \cong H_{Q}^{n+2}(E Q ; M)$, i.e., the cohomology of $Q$ is for large $n$ two-periodic with respect to any $\mathbb{Z} Q$-coefficient module $M$.

Remark 5.11. The inequality appearing in Theorem 5.8 (i) is sharp. For instance, we have $\operatorname{hdim}^{\mathbb{Z}^{n}}\left(\underline{E} \mathbb{Z}^{n}\right)=n$ and $\operatorname{hdim} \mathbb{Z}^{n}\left(\underline{\underline{E}} \mathbb{Z}^{n}\right)=n+1$ for $n \geq 2$ (see Example 5.21).

Example 5.12 (Counterexample to a conjecture of Connolly-Fehrmann-Hartglass). Let $G$ be the fundamental group of a closed hyperbolic manifold $M$ of dimension $d$. Then $G$ is torsionfree and satisfies $N M_{\mathcal{F} \mathcal{I N} \subseteq \mathcal{V} \mathcal{Y} Y}$ by Example 3.6. This implies, using Theorem 5.8 (ii)

$$
d=\operatorname{hdim}^{\{1\}}(M)=\operatorname{hdim}^{G}(E G)=\operatorname{hdim}^{G}(\underline{\underline{E}} G) .
$$

Obviously, $G$ is a discrete cocompact subgroup of the isometry group of the $d$ dimensional hyperbolic space, which is a Lie group with only finitely many path components.

Thus, we get counterexamples to a conjecture stated by Connolly-FehrmannHartglass [5, Conjecture 8.1], which predicts that $\operatorname{hdim}^{G}(\underline{\underline{E}} G)=d+1$. 


\section{$5.3 \quad$ Virtually poly- $\mathbb{Z}$ groups}

In this subsection, we give a complete calculation of $\operatorname{hdim}^{G}(\underline{\underline{E}} G)$ for virtually poly-ZZZ groups. Surprisingly, it will depend not only on $\operatorname{vcd}(G)=\operatorname{hdim}^{G}(\underline{E} G)$.

A group $G$ is poly- $\mathbb{Z}$ if there is a finite sequence $\{1\}=G_{0} \subseteq G_{1} \subseteq \ldots \subseteq$ $G_{n}=G$ of subgroups such that $G_{i-1}$ is normal in $G_{i}$ with infinite cyclic quotient $G_{i} / G_{i-1}$ for $i=1,2, \ldots, n$.

We have introduced notations like $[\mathcal{V C Y} \backslash \mathcal{F} \mathcal{I} \mathcal{N}],[V]$ and $N_{G}[V]$ at the beginning of Section 2. In particular, recall Definition 2.2 .

Theorem 5.13. Let $G$ be a virtually poly $\mathbb{Z}$ group which is not virtually cyclic. Let $[\mathcal{V C Y} \backslash \mathcal{F} \mathcal{I N}]_{f}$ be the subset of $[\mathcal{V C Y} \backslash \mathcal{F} \mathcal{I N}]$ consisting of those elements $[V]$ for which $N_{G}[V]$ has finite index in $G$. Let $[\mathcal{V C Y} \backslash \mathcal{F} \mathcal{I N}]_{f} / G$ be its quotient under the $G$-action coming from conjugation. Then

$$
\operatorname{hdim}^{G}(\underline{E} G)=\operatorname{vcd}(G),
$$

and precisely one of the following cases occurs:

(i) The set $[\mathcal{V C Y} \backslash \mathcal{F I N}]_{f} / G$ is empty. This is equivalent to the condition that every finite index subgroup of $G$ has a finite center. In this case,

$$
\operatorname{hdim}^{G}(\underline{\underline{E}} G)=\operatorname{vcd}(G)
$$

(ii) The set $[\mathcal{V C Y} \backslash \mathcal{F} \mathcal{I N}]_{f} / G$ contains exactly one element $[V] \cdot G$. This is equivalent to the condition that there exists an infinite normal cyclic subgroup $C \subseteq G$ and for every infinite cyclic subgroup $D \subseteq G$ with $\left[G: N_{G} D\right]<\infty$ we have $C \cap D \neq\{1\}$. Then there is the following dichotomy:

(a) For every $[W] \cdot G \in[\mathcal{V C Y} \backslash \mathcal{F I N}] / G$ such that $[W] \cdot G \neq[V] \cdot G$ we have $\operatorname{vcd}\left(N_{G}[W]\right) \leq \operatorname{vcd}(G)-2$. In this case, $\operatorname{vcd}(G) \geq 4$ and

$$
\operatorname{hdim}^{G}(\underline{\underline{E}} G)=\operatorname{vcd}(G)-1 ;
$$

(b) There exists $[W] \cdot G \in[\mathcal{V C Y} \backslash \mathcal{F} \mathcal{I N}] / G$ such that $[W] \cdot G \neq[V] \cdot G$ and $\operatorname{vcd}\left(N_{G}[W]\right)=\operatorname{vcd}(G)-1$. In this case, $\operatorname{vcd}(G) \geq 3$ and

$$
\operatorname{hdim}^{G}(\underline{\underline{E}} G)=\operatorname{vcd}(G) ;
$$


(iii) The set $[\mathcal{V C Y} \backslash \mathcal{F} \mathcal{I N}]_{f} / G$ contains more than one element. This is equivalent to the condition that there is a finite index subgroup of $G$ whose center contains a subgroup isomorphic to $\mathbb{Z}^{2}$. In this case,

$$
\operatorname{hdim}^{G}(\underline{\underline{E}} G)=\operatorname{vcd}(G)+1
$$

The proof of Theorem 5.13 needs some preparations.

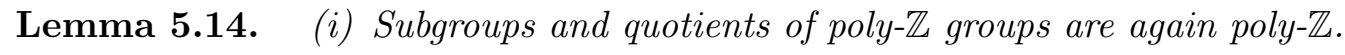
If $1 \rightarrow G_{0} \rightarrow G_{1} \rightarrow G_{2} \rightarrow 1$ is an extension of groups and $G_{0}$ and $G_{2}$ are poly $\mathbb{Z}$ groups, then $G$ is a poly- $\mathbb{Z}$ group.

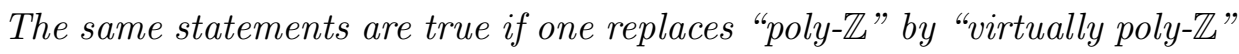
everywhere;

(ii) Let $G$ be a poly- $\mathbb{Z}$ group of cohomological dimension $\operatorname{cd}(G)=d$. Then $H^{d}(G)$ is isomorphic to $\mathbb{Z}$ or to $\mathbb{Z} / 2$;

(iii) Let $G$ be a poly- $\mathbb{Z}$ group. Suppose that there is a finite sequence $\{1\}=$ $G_{0} \subseteq G_{1} \subseteq \ldots \subseteq G_{n}=G$ of subgroups such that $G_{i-1}$ is normal in $G_{i}$ with infinite cyclic quotient $G_{i} / G_{i-1}$ for $i=1,2, \ldots, n$. Then

$$
n=\operatorname{cd}(G)
$$

(iv) If $1 \rightarrow G_{0} \rightarrow G_{1} \rightarrow G_{2} \rightarrow 1$ is an extension of virtually poly-ZZZ groups, then

$$
\operatorname{vcd}\left(G_{1}\right)=\operatorname{vcd}\left(G_{0}\right)+\operatorname{vcd}\left(G_{2}\right)
$$

(v) A virtually poly-ZZZ group $G$ has the following property:

If $\phi: G \rightarrow G$ is an automorphism, $g \in G$ is an element

(A) of infinite order and $a, b \in \mathbb{Z}$ are integers such that $\phi\left(g^{a}\right)=g^{b}$, then $a= \pm b$.

Proof. (i) The elementary proof of this assertion is left to the reader.

(ii) We use induction over the number $n$ for which there is a finite sequence $\{1\}=G_{0} \subseteq G_{1} \subseteq \ldots \subseteq G_{n}=G$ of subgroups such that $G_{i-1}$ is normal in $G_{i}$ with infinite cyclic quotient $G_{i} / G_{i-1}$ for $i=1,2, \ldots, n$. If $n=0$, then $G$ is trivial 
and the claim is obviously true. The induction step from $n-1$ to $n \geq 1$ is done as follows.

By the induction hypothesis, the claim is true for $G_{n-1}$. This means that $\operatorname{cd}\left(G_{n-1}\right)=n-1$, that $H^{n-1}\left(G_{n-1}\right)$ is isomorphic to $\mathbb{Z}$ or $\mathbb{Z} / 2$ and that $H^{i}\left(G_{n-1}\right)=$ 0 for $i \geq n$. We have the extension $1 \rightarrow G_{n-1} \rightarrow G \rightarrow \mathbb{Z} \rightarrow 1$ if we identify $G / G_{n-1}$ with $\mathbb{Z}$. Let $f: G_{n-1} \rightarrow G_{n}$ be the group automorphism induced by conjugation with some preimage in $G$ of a generator of $\mathbb{Z}$. The associated Hochschild-Serre cohomology spectral sequence yields the exact Wang sequence

$$
H^{n-1}\left(G_{n-1}\right) \stackrel{\text { id }-H^{n-1}(f)}{\longrightarrow} H^{n-1}\left(G_{n-1}\right) \rightarrow H^{n}(G) \rightarrow H^{n}\left(G_{n-1}\right)=0 .
$$

Since $H^{n-1}\left(G_{n-1}\right)$ is isomorphic to $\mathbb{Z}$ or $\mathbb{Z} / 2$, the automorphism $H^{n-1}(f)$ is \pm id. Hence $H^{n}(G) \cong \mathbb{Z}$ if and only if $H^{n-1}\left(G_{n-1}\right) \cong \mathbb{Z}$ and $H^{n-1}(f)=$ id, and $H^{n}(G) \cong \mathbb{Z} / 2$ if and only if either $H^{n-1}\left(G_{n-1}\right) \cong \mathbb{Z}$ and $H^{n-1}(f)=-$ id or $H^{n-1}\left(G_{n-1}\right) \cong \mathbb{Z} / 2$.

(iii) We use induction over the number $n$ as above. Since $H^{n}(G)$ is non-trivial, we have $\operatorname{cd}(G) \geq n$. On the other hand, the cohomological dimension is subadditive under extensions, i.e., $\operatorname{cd}(G) \leq \operatorname{cd}\left(G_{n-1}\right)+\operatorname{cd}(\mathbb{Z})=(n-1)+1=n$. We conclude $\operatorname{cd}(G)=n$.

(iv) One easily constructs an exact sequence of poly- $\mathbb{Z}$ groups $1 \rightarrow G_{0}^{\prime} \rightarrow G_{1}^{\prime} \rightarrow$ $G_{2}^{\prime} \rightarrow 1$ such that $G_{i}^{\prime}$ is a subgroup of finite index in $G_{i}$ for $i=0,1,2$. Now, it suffices to show that $\operatorname{cd}\left(G_{1}^{\prime}\right)=\operatorname{cd}\left(G_{0}^{\prime}\right)+\operatorname{cd}\left(G_{2}^{\prime}\right)$. By induction over the number $n$ as above but with $G_{2}^{\prime}$ in place of $G$, the claim reduces to the case that $G_{2}^{\prime}=\mathbb{Z}$, which follows from assertion (iii).

(v) Consider a group extension $1 \rightarrow G_{0} \stackrel{i}{\rightarrow} G_{1} \stackrel{p}{\rightarrow} G_{2} \rightarrow 1$ such that $G_{0}$ is characteristic in $G_{1}$ and $G_{0}$ and $G_{2}$ have property $(A)$. Then $G_{1}$ has property $(A)$ by the following argument. Consider an automorphism $\phi_{1}: G_{1} \rightarrow G_{1}$. It induces automorphisms $\phi_{0}: G_{0} \rightarrow G_{0}$ and $\phi_{2}: G_{2} \rightarrow G_{2}$ satisfying $\phi_{1} \circ i=i \circ \phi_{0}$ and $\phi_{2} \circ p=p \circ \phi_{1}$ since $G_{0}$ is characteristic by assumption. Let $g \in G$ be of infinite order and $a, b \in \mathbb{Z}$ such that $\phi_{1}\left(g^{a}\right)=g^{b}$. Then $\phi_{2}\left(p(g)^{a}\right)=p(g)^{b}$, and if $p(g)$ has infinite order, we see that $a= \pm b$ since $G_{2}$ has property $(A)$ by assumption. If $p(g)$ has finite order, we can choose $c \in \mathbb{Z}, c \neq 0$ such that $g^{c} \in G_{0}$. Then $\phi_{1}\left(g^{a}\right)=g^{b}$ implies $\phi_{0}\left(\left(g^{c}\right)^{a}\right)=\left(g^{c}\right)^{b}$ in $G_{0}$ and hence $a= \pm b$ since $G_{0}$ has property $(A)$ by assumption. Hence, $G_{1}$ has property $(A)$. 
Now we prove by induction over $\operatorname{vcd}(G)$ that a virtually poly- $\mathbb{Z}$ group $G$ has property $(A)$. The induction beginning $\operatorname{vcd}(G)=0$ is trivial since then $G$ is finite. The induction step is done as follows. Let $K \subseteq G$ be a subgroup of finite index such that $K$ is poly- $\mathbb{Z}$. Then $K_{0}=\bigcap\{\phi(K) \mid \phi \in \operatorname{aut}(G)\}$ is a characteristic subgroup of $G$. Since $G$ is finitely generated and, thus, contains only finitely many subgroups of any given index, the intersection defining $K_{0}$ is an intersection of finitely many groups of finite index. Hence, $K_{0}$ is a characteristic subgroup of finite index in $G$. Since every finite group has property $(A)$, the group $G$ has property $(A)$ if $K_{0}$ has. Consider the group extension $1 \rightarrow K_{1} \rightarrow$ $K_{0} \rightarrow H_{1}\left(K_{0}\right) / \operatorname{tors}\left(H_{1}\left(K_{0}\right)\right) \rightarrow 1$. Obviously, $K_{1}$ is a characteristic subgroup of $K_{0}$. Since $K_{0}$, being a subgroup of the poly- $\mathbb{Z}$ group $K$, is poly- $\mathbb{Z}$, the abelian group $H_{1}\left(K_{0}\right) / \operatorname{tors}\left(H_{1}\left(K_{0}\right)\right.$ is isomorphic to $\mathbb{Z}^{n}$ for some $n \geq 1$. This implies that $K_{1}$ is a poly- $\mathbb{Z}$ group satisfying $\operatorname{vcd}\left(K_{1}\right)<\operatorname{vcd}\left(K_{0}\right)=\operatorname{vcd}(G)$. By induction hypothesis, $K_{1}$ has property $(A)$. Clearly, $\mathbb{Z}^{n}$ also has property $(A)$. This implies that $K_{0}$ and hence $G$ have property $(A)$. This finishes the proof of Lemma 5.14.

The statements about the top homology of a poly- $\mathbb{Z}$ group $G$ appearing in Lemma 5.14 would be obvious if there were closed manifold models for $B G$. Instead of dealing with this difficult question we have preferred to give an easy homological argument.

Lemma 5.15. Let $G$ be a virtually poly- $\mathbb{Z}$ group. Let $V \subseteq G$ be an infinite virtually cyclic group. Then we can find an infinite cyclic subgroup $C \subseteq V$ such that $[V]=[C] \in[\mathcal{V C Y} \backslash \mathcal{F I N}]$ and $N_{G} C=N_{G}[V]$ holds, and such that a model for $E_{\mathcal{V C Y}[V]}\left(N_{G}[V]\right)$ is given by $\underline{E} W_{G} C$ considered as an $N_{G}[V]=N_{G} C-C W$ complex by restriction with the canonical projection $N_{G} C \rightarrow W_{G} C$.

Proof. We can assume that $V$ itself is infinite cyclic (otherwise, we pass to a subgroup of finite index). Since $N_{G}[V]$ is a subgroup of the virtually poly- $\mathbb{Z}$ group $G$, it is virtually poly- $\mathbb{Z}$ and, in particular, finitely generated. Let $\left\{g_{1}, g_{2}, \ldots, g_{r}\right\}$ be a finite generating set. Fix a generator $c \in V$. Since $g_{i} \in N_{G}[V]$, we can find integers $a_{i}, b_{i} \in \mathbb{Z}$ different from zero such that $g_{i} c^{a_{i}} g_{i}^{-1}=c^{b_{i}}$. Property $(A)$ (see Lemma $5.14(\mathrm{v}))$ implies $b_{i}= \pm a_{i}$. Put $a=a_{1} a_{2} \cdots a_{r}$. Then $g_{i} c^{a} g_{i}^{-1}=c^{ \pm a}$ holds for $i=1,2, \ldots, r$. Let $C$ be the infinite cyclic subgroup of $V$ generated by 
$c^{a}$. Then, of course, $[C]=[V]$. Furthermore, $g_{i} \in N_{G} C$ for $i=1,2, \ldots, r$, which implies $N_{G}[V] \subseteq N_{G} C$. Hence, $N_{G}[V]=N_{G} C$.

It remains to prove that $\underline{E} W_{G} C$ is a model for $E_{\mathcal{V C Y}[C]}\left(N_{G} C\right)$. This follows from the fact that, under the projection $N_{G} C \rightarrow W_{G} C$, the family of all virtually cyclic subgroups of $N_{G} C$ whose intersection with $C$ is infinite coincides with the family of all finite subgroups of $W_{G} C$.

Now we are ready to prove Theorem 5.13.

Proof. Because of Lemma 5.15, we can choose a set $I$ of infinite cyclic subgroups of $G$ and models for $\underline{E} N_{G} C$ and $\underline{E} W_{G} C$ for $C \in I$ such that the following properties hold:

- For every element $\eta \in[\mathcal{V C Y} \backslash \mathcal{F I N}] / G$, there exists precisely one $C \in I$ such that $[C] \cdot G=\eta$;

- $N_{G}[C]=N_{G} C$ for all $C \in I$;

- For all $C \in I$, a model for $E_{\mathcal{V C Y}[C]}\left(N_{G}[C]\right)$ is given by $\underline{E} W_{G} C$, considered as an $N_{G} C$-CW-complex by restriction with the canonical projection $N_{G} C \rightarrow$ $W_{G} C$.

From Theorem 2.3 we obtain a $G$-pushout

$$
\begin{aligned}
& \coprod_{C \in I} G \times_{N_{G} C} \underline{E} N_{G} C \stackrel{i}{\longrightarrow} \underline{E} G \\
& \downarrow \amalg_{C \in I} \operatorname{id}_{G} \times_{N_{G} C} f_{C} \downarrow \\
& \amalg_{C \in I} G \times_{N_{G} C} \underline{E} W_{G} C \longrightarrow \stackrel{\llcorner}{\underline{E}} G
\end{aligned}
$$

such that $f_{C}$ is a cellular $N_{G} C$-map for every $C \in I$ and $i$ is an inclusion of $G$ - $C W$-complexes.

In the remainder of the proof, we will frequently use that Lemma 5.14 (iv) implies

$$
\begin{aligned}
\operatorname{vcd}\left(W_{G} C\right) & =\operatorname{vcd}\left(N_{G} C\right)-1 ; \\
\operatorname{vcd}\left(N_{G} C\right) & \leq \operatorname{vcd}(G) ; \\
\operatorname{vcd}\left(N_{G} C\right)=\operatorname{vcd}(G) & \Leftrightarrow\left[G: N_{G} C\right]<\infty .
\end{aligned}
$$


Furthermore, we conclude from [16, Example 5.26 on page 305] that

$$
\begin{aligned}
\operatorname{hdim}^{G}(\underline{E} G) & =\operatorname{vcd}(G) ; & & \\
\operatorname{hdim}^{G}\left(\underline{E} N_{G} C\right) & =\operatorname{vcd}\left(N_{G} C\right) & & \text { for } C \in I ; \\
\operatorname{hdim}^{G}\left(\underline{E} W_{G} C\right) & =\operatorname{vcd}\left(N_{G} C\right)-1 & & \text { for } C \in I .
\end{aligned}
$$

(i) As $[\mathcal{V C Y} \backslash \mathcal{F} \mathcal{I N}]_{f} / G=\emptyset$, we must have $\operatorname{hdim}^{G}\left(\underline{E} W_{G} C\right)<\operatorname{hdim}^{G}\left(\underline{E} N_{G} C\right)<$ $\operatorname{vcd}(G)$, whereas $\operatorname{hdim}^{G}(\underline{E} G)=\operatorname{vcd}(G)$. Hence, we conclude from the $G$-pushout (5.16) together with Remark 2.5 that hdim${ }^{G}(\underline{\underline{E}} G) \leq \operatorname{vcd}(G)$. In order to show hdim ${ }^{G}(\underline{\underline{E}} G) \geq$ $\operatorname{vcd}(G)$ we can assume without loss of generality that $G$ is poly- $\mathbb{Z}$ since there is a poly- $\mathbb{Z}$ subgroup $G^{\prime}$ of finite index and the restriction of $\underline{\underline{E}} G$ to $G^{\prime}$ is a model for $\underline{\underline{E}} G^{\prime}$. The $G$-pushout (5.16) yields a pushout of $C W$-complexes

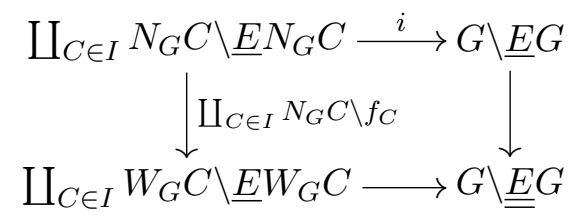

From the associated long exact Mayer-Vietoris sequence, we obtain an epimorphism $H^{\operatorname{vcd}(G)}(G \backslash \underline{\underline{E}} G) \rightarrow H^{\operatorname{vcd}(G)}(G \backslash \underline{E} G)$. However, $H^{\operatorname{vcd}(G)}(G \backslash \underline{E} G)$ is nontrivial by Lemma 5.14 (ii), so $H^{\operatorname{vcd}(G)}(G \backslash \underline{\underline{E}} G)$ is also non-trivial. This means that $\operatorname{hdim}^{G}(\underline{\underline{E}} G) \geq \operatorname{vcd}(G)$.

As $C_{G} C$ has finite index in $N_{G} C$ for $C \in I$, the condition $[\mathcal{V C Y} \backslash \mathcal{F} \mathcal{I N}]_{f} / G=\emptyset$ is equivalent to the condition that for every infinite cyclic subgroup $C \subseteq G$ we have $\left[G: C_{G} C\right]=\infty$. Obviously, this is true if and only if the center of any finite index subgroup of $G$ is finite. This finishes the proof of assertion (i).

(iii) We conclude from the $G$-pushout (5.16) together with Remark 2.5 that $\operatorname{hdim}^{G}(\underline{\underline{E}} G) \leq \operatorname{vcd}(G)+1$ as $\operatorname{hdim}^{G}\left(\underline{E} N_{G} C\right), \operatorname{hdim}^{G}\left(\underline{E} W_{G} C\right)$ and $\operatorname{hdim}^{G}(\underline{E} G)$ are less or equal to $\operatorname{vcd}(G)$. It remains to show that $\operatorname{hdim}^{G}(\underline{\underline{E}} G) \geq \operatorname{vcd}(G)+1$, and it suffices to do this for a subgroup of finite index. Hence, we can assume without loss of generality that $G$ is poly- $\mathbb{Z}$. We obtain from the pushout (5.17) the short exact sequence

$$
H^{\operatorname{vcd}(G)}(G \backslash \underline{E} G) \rightarrow \prod_{C \in I} H^{\operatorname{vcd}(G)}\left(N_{G} C \backslash \underline{E} N_{G} C\right) \rightarrow H^{\operatorname{vcd}(G)+1}(G \backslash \underline{\underline{E}} G) \rightarrow 0 .
$$


If we can show that $H^{\operatorname{vcd}(G)}(G \backslash \underline{E} G) \rightarrow \prod_{C \in I} H^{\operatorname{vcd}(G)}\left(N_{G} C \backslash \underline{E} N_{G} C\right)$ is not surjective, then $H^{\operatorname{vcd}(G)+1}(G \backslash \underline{\underline{E}} G)$ is non-trivial, implying $\operatorname{hdim}^{G}(\underline{\underline{E}} G) \geq \operatorname{vcd}(G)+1$. By Lemma 5.14 (ii), $H^{\operatorname{vcd}(G)}(G \backslash \underline{E} G)$ and $H^{\operatorname{vcd}\left(N_{G} C\right)}\left(N_{G} C \backslash \underline{E} N_{G} C\right)$ are nontrivial cyclic groups. Thus, $H^{\operatorname{vcd}(G)}(G \backslash \underline{E} G) \rightarrow \prod_{C \in I} H^{\operatorname{vcd}(G)}\left(N_{G} C \backslash \underline{E} N_{G} C\right)$ cannot be surjective if there is more than one element $C \in I$ with $\operatorname{vcd}\left(N_{G} C\right)=$ $\operatorname{vcd}(G)$, which is indeed the case here since, by assumption, $[\mathcal{V C Y} \backslash \mathcal{F} \mathcal{I N}]_{f} / G$ contains more than one element.

We still want to show that this latter condition is equivalent to the condition that there is a finite index subgroup of $G$ whose center contains a copy of $\mathbb{Z}^{2}$. Suppose first that $[\mathcal{V C Y} \backslash \mathcal{F} \mathcal{I N}]_{f} / G$ contains two different elements $\left[V_{1}\right] \cdot G$ and $\left[V_{2}\right] \cdot G$. Lemma 5.15 shows that we may assume that $V_{i}$ is infinite cyclic and $N_{G} V_{i}=N_{G}\left[V_{i}\right]$ for $i=1,2$. Then, as $C_{G} V_{i}$ has finite index in $N_{G} V_{i}$ for $i=1,2$, the subgroup $C_{G} V_{1} \cap C_{G} V_{2} \subseteq G$ is also of finite index. Choose infinite cyclic groups $C_{1}$ and $C_{2}$ such that $C_{1} \subseteq V_{1} \cap C_{G} V_{2}$ and $C_{2} \subseteq V_{2} \cap C_{G} V_{1}$, and let $H \subseteq G$ be the subgroup generated by $C_{1}$ and $C_{2}$. Then $C_{G} H$ contains $C_{G} V_{1} \cap C_{G} V_{2}$. Hence $C_{G} H$ has finite index in $G$ and $H \subseteq C_{G} H$. In particular, $H$ is abelian. Since $\left[V_{1}\right] \neq\left[V_{2}\right]$, we have $C_{1} \cap C_{2}=\{1\}$. So $H$ is isomorphic to $\mathbb{Z}^{2}$, and we have found a finite index subgroup of $G$, namely $C_{G} H$, whose center contains $\mathbb{Z}^{2}$.

Conversely, suppose that $G^{\prime} \subseteq G$ is a subgroup of finite index whose center contains $\mathbb{Z}^{2}$. By passing to a subgroup of finite index in $G^{\prime}$, we can arrange for $G^{\prime} \subseteq G$ to be torsionfree and normal with finite quotient $G / G^{\prime}$. Since $\operatorname{cent}\left(G^{\prime}\right)$ is isomorphic to $\mathbb{Z}^{n}$ for some $n \geq 2$, it contains infinitely many maximal infinite cyclic subgroups. As $G / G^{\prime}$ is finite, $[\mathcal{V C Y} \backslash \mathcal{F} \mathcal{I N}]_{f} / G$ must be infinite and, in particular, contains more than one element.

(ii) We claim that $[\mathcal{V C Y} \backslash \mathcal{F} \mathcal{I N}]_{f} / G$ consists of precisely one element if and only if $G$ contains an infinite cyclic normal subgroup $C$ and for every infinite cyclic subgroup $D \subseteq G$ with $\left[G: N_{G} D\right]<\infty$ we have $C \cap D \neq\{1\}$. The "if"-statement is obvious, so let us show that $G$ contains an infinite cyclic normal subgroup if $[\mathcal{V C Y} \backslash \mathcal{F} \mathcal{I N}]_{f} / G$ consists of precisely one element $[V] \cdot G$. Again, just as in the proof of (iii), we can assume by Lemma 5.15 that $V$ is infinite cyclic and $\left[G: C_{G} V\right]<\infty$. This implies that $K:=\bigcap_{g \in G} g C_{G} V g^{-1}$ is a normal subgroup of $G$ of finite index. Thus, the center $\operatorname{cent}(K)$ of $K$, being a characteristic subgroup of $K$, is also normal in $G$. Moreover, we conclude from assertion (iii) that cent $(K)$ 
does not contain $\mathbb{Z}^{2}$ as subgroup of finite index. Hence, it is isomorphic to $\mathbb{Z} \times A$ for some finite abelian subgroup $A$. If $a$ is the order of $A$, then $C:=\{a \cdot x \mid x \in$ $\operatorname{cent}(K)\}$ is a characteristic infinite cyclic subgroup of $\operatorname{cent}(K)$ and hence normal in $G$. The claim follows.

In the remainder, let $C_{0} \subseteq G$ be an infinite cyclic normal subgroup. This means that $[\mathcal{V C Y} \backslash \mathcal{F} \mathcal{I N}]_{f} / G=\left\{\left[C_{0}\right] \cdot G\right\}$. Since we assume that $G$ is not virtually cyclic, $\operatorname{vcd}(G) \geq 2$. In fact, we have $\operatorname{vcd}(G)>2$. Namely, if $\operatorname{vcd}(G)=2$, then $G$ contains a subgroup of finite index isomorphic to $\mathbb{Z}^{2}$, which is only possible in the case (iii) and not in the case (ii) under consideration.

Next, we want to show that $\operatorname{vcd}(G) \geq 4$ in the case (ii)(a) and $\operatorname{vcd}(G) \geq 3$ in the case (ii)(b). We can assume that $C_{0} \subseteq G$ is central and that $G$ is poly- $\mathbb{Z}$ for, otherwise, we can replace $G$ by a subgroup of finite index. Choose an element $g \in G$ whose image under the projection $G \rightarrow G / C_{0}$ is an element of infinite order. Let $D$ be the cyclic subgroup generated by $g \in G$. Then $D \cap C_{0}=\{1\}$ and $N_{G} D$ contains a subgroup isomorphic to $\mathbb{Z}^{2}$. Hence $\operatorname{vcd}\left(N_{G} D\right) \geq 2$ and $[D] \neq\left[C_{0}\right]$. The assumptions imply $\operatorname{vcd}(D) \leq \operatorname{vcd}(G)-2$, which in turn implies $\operatorname{vcd}(G) \geq 4$ in the case (ii)(a) and $\operatorname{vcd}(G) \geq 3$ in the case (ii)(b).

In order to get a model for $\underline{\underline{E}} G$ of the desired dimension, we have to investigate the $G$-pushout (5.16) more closely and use some machinery. Let $\underline{\underline{E}} G$ be the $G$ - $C W$-model obtained from the $G$-pushout (5.16). Given a natural number $d \geq 3$, we will show that there exists a $G$-CW-complex of dimension $d$ which is $G$-homotopy equivalent to $\underline{\underline{E}} G$ by checking that for every contravariant $\mathbb{Z}$ Or $(G)$-module $M$ the Bredon cohomology $H_{\mathbb{Z} \operatorname{Or}(G)}^{d+1}(\underline{\underline{E}} G ; M)$ vanishes and for every $i \geq d+1$ the homology of $\underline{\underline{E}} G^{H}$ vanishes for $H \subseteq G$ (see [13, Proposition 11.10 on page 221 and Theorem 13.19 on page 268]). The latter condition is obviously satisfied since $\underline{\underline{E}} G^{H}$ is contractible or empty. It remains to prove $H_{\mathbb{Z} \operatorname{Or}(G)}^{d+1}(\underline{\underline{E}} G ; M)=0$.

Let $C \in I$ be the unique element for which $\operatorname{vcd}\left(N_{G} C\right)=\operatorname{vcd}(G)$. After replacing $C$ by $C_{0}$, we can actually assume that $N_{G} C=G$. Let us put $d=\operatorname{vcd}(G)$ if $\operatorname{vcd}\left(N_{G} D\right)=\operatorname{vcd}(G)-1$ for at least one $D \in I$ with $D \neq C$ and $d=\operatorname{vcd}(G)-1$ if $\operatorname{vcd}\left(N_{G} D\right) \leq \operatorname{vcd}(G)-2$ holds for all $D \in I$ with $D \neq C$. Then we have $d \geq 3$ and, in all cases, $\operatorname{vcd}\left(N_{G} D\right)<d$ for all $D \in I$ with $D \neq C$. In particular, 
$H_{\mathbb{Z} \operatorname{Or}(G)}^{k}\left(G \times_{N_{G} D} \underline{E} N_{G} D ; M\right)$ as well as $H_{\mathbb{Z} \operatorname{Or}(G)}^{k}\left(G \times_{N_{G} D} \underline{E} W_{G} D ; M\right)$ vanish for all $D \in I$ with $D \neq C$ if $k \geq d$. The long exact Mayer-Vietoris sequence for the Bredon cohomology associated to the $G$-pushout (5.16) yields the exact sequence

$$
\begin{aligned}
H_{\mathbb{Z} \mathrm{Or}(G)}^{d}(\underline{E} G ; M) \stackrel{\mathrm{id}}{\rightarrow} H_{\mathbb{Z} \mathrm{Or}(G)}^{d}(\underline{E} G ; M) \rightarrow H_{\mathbb{Z} \mathrm{Or}(G)}^{d+1}(\underline{\underline{E}} G ; M) \\
\rightarrow H_{\mathbb{Z} \mathrm{Or}(G)}^{d+1}(\underline{E} G ; M) \stackrel{\mathrm{id}}{\longrightarrow} H_{\mathbb{Z} \mathrm{Or}(G)}^{d+1}(\underline{E} G ; M) .
\end{aligned}
$$

Hence, $H_{\mathbb{Z} \operatorname{Or}(G)}^{d+1}(\underline{\underline{E}} G ; M)$ is trivial and, as explained above, we can find a $d$ dimensional $G$ - $C W$-model for $\underline{\underline{E}} G$. We conclude that $\operatorname{hdim}^{G}(\underline{\underline{E}} G) \leq \operatorname{vcd}(G)-1$ in the case (ii)(a) and $\operatorname{hdim}^{G}(\underline{\underline{E}} G) \leq \operatorname{vcd}(G)$ in the case (ii)(b).

Finally, we prove $\operatorname{hdim}^{G}(\underline{\underline{E}} G) \geq \operatorname{vcd}(G)-1$ in the case (ii)(a) as well as $\operatorname{hdim}^{G}(\underline{\underline{E}} G) \geq \operatorname{vcd}(G)$ in the case (ii)(b). The first inequality follows from Corollary 5.4. Concerning the second, we can assume that $G$ is poly- $\mathbb{Z}$. Consider the long exact Mayer-Vietoris sequence associated to the pushout (5.17). Since a poly-ZZ-group is torsionfree, $N_{G} C=G$ and $\operatorname{cd}\left(E W_{G} D\right) \leq d-2$ for $D \in I$ with $D \neq C$, it yields the long exact sequence

$$
\begin{aligned}
H^{d-1}(G) \stackrel{\operatorname{id} \times j}{\longrightarrow} H^{d-1}(G) \times \prod_{D \in I, D \neq C} H^{d-1}\left(N_{G} D\right) \rightarrow H^{d}(G \backslash \underline{\underline{E}} G) & \\
& \rightarrow H^{d}(G) \stackrel{\mathrm{id}}{\longrightarrow} H^{d}(G) .
\end{aligned}
$$

As $H^{d-1}\left(N_{G} D\right)$ is non-trivial if $D \in I$ is such that $\operatorname{vcd}\left(N_{G} D\right)=d-1$ (see Lemma 5.14 (ii)), we conclude $H^{d}(G \backslash \underline{\underline{E}} G) \neq 0$ and hence $\operatorname{hdim}^{G}(\underline{\underline{E}} G) \geq d=$ $\operatorname{vcd}(G)$. This finishes the proof of Theorem 5.13.

Corollary 5.18. Let $G$ be a virtually poly- $\mathbb{Z}$ group. Suppose that the group $G^{\prime}$ is a subgroup of $G$ of finite index or that $G^{\prime}$ is the quotient of $G$ by a finite normal subgroup. Then

$$
\begin{aligned}
& \operatorname{hdim}^{G^{\prime}}\left(\underline{E} G^{\prime}\right)=\operatorname{hdim}^{G}(\underline{E} G) ; \\
& \operatorname{hdim}^{G^{\prime}}\left(\underline{\underline{E}} G^{\prime}\right)=\operatorname{hdim}^{G}(\underline{\underline{E}} G) .
\end{aligned}
$$

Proof. We get $\operatorname{vcd}\left(G^{\prime}\right)=\operatorname{vcd}(G)$ from Lemma 5.14 (iv). Hence, $\operatorname{hdim}^{G^{\prime}}\left(\underline{E} G^{\prime}\right)=$ $\operatorname{hdim}^{G}(\underline{E} G)$ follows immediately from Theorem 5.13.

The second assertion of the corollary will follow from assertions (i), (ii) and (iii) of Theorem 5.13. We only need to show that every subgroup of $G^{\prime}$ of finite index 
has a finite center if and only if every subgroup of $G$ of finite index has a finite center and that $G^{\prime}$ contains a finite index subgroup whose center contains $\mathbb{Z}^{2}$ if and only if $G$ contains a finite index subgroup whose center contains $\mathbb{Z}^{2}$.

We begin with the case that $G^{\prime}$ is a subgroup of finite index in $G$. A finitely generated abelian group $A$ satisfies $\operatorname{vcd}(A) \geq 2$ if and only if it contains a subgroup isomorphic to $\mathbb{Z}^{2}$. Obviously, a subgroup $H \subseteq G$ satisfies $[G: H]<\infty$ if and only if $\left[G^{\prime}:\left(G^{\prime} \cap H\right)\right]<\infty$. Now the claims follow from the observation that for a subgroup $H \subseteq G$ we have $\operatorname{cent}(H) \cap G^{\prime} \subseteq \operatorname{cent}\left(H \cap G^{\prime}\right)$ and $[\operatorname{cent}(H)$ : $\left.\left(\operatorname{cent}(H) \cap G^{\prime}\right)\right]<\infty$, which implies that $\operatorname{vcd}(\operatorname{cent}(H)) \leq \operatorname{vcd}\left(\operatorname{cent}\left(H \cap G^{\prime}\right)\right)$ holds.

It remains to treat the case that there is an extension $1 \rightarrow F \rightarrow G \stackrel{p}{\rightarrow} G^{\prime} \rightarrow 1$ in which the group $F$ is finite. Let $K \subseteq G$ be a subgroup, then $p(\operatorname{cent}(K)) \subseteq$ $\operatorname{cent}(p(K))$, which implies

$$
\operatorname{vcd}(\operatorname{cent}(K)) \leq \operatorname{vcd}(\operatorname{cent}(p(K))) .
$$

Let $H \subseteq G^{\prime}$ be a subgroup. Since $G$ is virtually poly- $\mathbb{Z}$, by Lemma 5.14 (i) the same is true for the subgroup $p^{-1}(H)$. In particular, $p^{-1}(H)$ is finitely generated, say by the finite set $\left\{s_{1}, s_{2}, \ldots, s_{r}\right\}$. Consider the map

$$
\gamma: p^{-1}(\operatorname{cent}(H)) \rightarrow \prod_{i=1}^{r} F, \quad h \mapsto\left\{\left[h, s_{i}\right] \mid i=1,2, \ldots r\right\} .
$$

We have $p\left(\operatorname{cent}\left(p^{-1}(H)\right) \subseteq \operatorname{cent}\left(p\left(p^{-1}(H)\right)=\operatorname{cent}(H)\right.\right.$ and hence $\operatorname{cent}\left(p^{-1}(H)\right)$ is a subgroup of $p^{-1}(\operatorname{cent}(H))$. Now one easily checks that $\gamma$ induces an injection $p^{-1}(\operatorname{cent}(H)) / \operatorname{cent}\left(p^{-1}(H)\right) \rightarrow \prod_{i=1}^{r} F$, which means that $\operatorname{cent}\left(p^{-1}(H)\right)$ has finite index in $p^{-1}(\operatorname{cent}(H))$. As $F$ is finite, we conclude that

$$
\operatorname{vcd}\left(\operatorname{cent}\left(p^{-1}(H)\right)\right)=\operatorname{vcd}(\operatorname{cent}(H)) \text {. }
$$

Obviously, $[G: K]<\infty$ if and only if $\left[G^{\prime}: p(K)\right]<\infty$, and $\left[G^{\prime}: H\right]<\infty$ if and only if $\left[G: p^{-1}(H)\right]<\infty$. Thus, the claim follows from (5.19) and (5.20).

\subsection{Extensions of free abelian groups}

The following is a consequence of Theorem 5.13 (iii) and Corollary 5.18. It has already been proved for crystallographic groups by Connolly-Fehrmann-Hartglass [5]. 
Example 5.21 (Virtually $\mathbb{Z}^{n}$-groups). Let $G$ be a group which contains $\mathbb{Z}^{n}$ as subgroup of finite index. If $n \geq 2$, then

$$
\operatorname{hdim}^{G}(\underline{\underline{E}} G)=n+1
$$

If $n \leq 1$, then $\operatorname{hdim}^{G}(\underline{\underline{E}} G)=0$.

Theorem 5.22. Let $1 \rightarrow \mathbb{Z}^{n} \rightarrow G \stackrel{p}{\rightarrow} \mathbb{Z} \rightarrow 1$ be a group extension for $n \geq 2$. Let $\phi: \mathbb{Z}^{n} \rightarrow \mathbb{Z}^{n}$ be the automorphism given by conjugation with some $g \in G$ which is mapped to a generator of $\mathbb{Z}$ under $p$.

Then there is the following dichotomy:

(i) We have $\operatorname{cd}\left(\operatorname{ker}\left(\phi^{k}-\mathrm{id}\right)\right) \leq 1$ for all $k \in \mathbb{Z}$. In this case,

$$
\operatorname{hdim}^{G}(\underline{\underline{E}} G)=n+1
$$

(ii) We have $\operatorname{cd}\left(\operatorname{ker}\left(\phi^{k}-\mathrm{id}\right)\right) \geq 2$ for some $k \in \mathbb{Z}$. In this case,

$$
\operatorname{hdim}^{G}(\underline{\underline{E}} G)=n+2 .
$$

Proof. We first deal with the case that $\phi$ is periodic, i.e., there exists $k \geq 1$ such that $\phi^{k}=$ id. Since $n \geq 2$, we are then in the situation of case (ii). The group $G$ contains a subgroup of finite index which is isomorphic to $\mathbb{Z}^{n+1}$ and, hence, $\operatorname{hdim}^{G}(\underline{\underline{E}} G)=n+2$ by Example 5.21. Thus, we can assume in the remainder that $\phi$ is not periodic.

Next, we show that the epimorphism $p: G \rightarrow \mathbb{Z}$ satisfies $p(V)=0$ for all $[V] \cdot G \in[\mathcal{V C Y} \backslash \mathcal{F} \mathcal{I N}]_{f} / G$. Suppose to the contrary that there exists $[V] \in$ $[\mathcal{V C Y} \backslash \mathcal{F} \mathcal{I N}]$ such that $N_{G}[V]$ has finite index in $G$ and $p(V) \neq 0$. Because of Lemma 5.15, we can assume without loss of generality that $V$ is infinite cyclic and $N_{G}[V]=N_{G} V$. Since $C_{G} V$ has finite index in $N_{G} V$, we conclude that $C_{G} V$ has finite index in $G$. Let $v \in V$ be a generator of $V$. Then $\phi^{p(v)}: \mathbb{Z}^{n} \rightarrow \mathbb{Z}^{n}$ is given by conjugation with $v$ and, hence, is the identity on the subgroup $\mathbb{Z}^{n} \cap C_{G} V$ of finite index in $\mathbb{Z}^{n}$. This shows that $\phi^{p(v)}=\mathrm{id}$, contradicting the assumption that $\phi$ is not periodic. 
Now, consider $[V] \cdot G \in[\mathcal{V C} \mathcal{Y} \backslash \mathcal{F} \mathcal{I} \mathcal{N}]_{f} / G$. Then $p(V)=0$ implies $\mathbb{Z}^{n} \subseteq N_{G}[V]$ and $N_{G}[V]=p^{-1}\left(p\left(N_{G}[V]\right)\right)$. One easily checks that

$$
p\left(N_{G}[V]\right)=\left\{k \in \mathbb{Z} \mid V \subseteq \operatorname{ker}\left(\phi^{k}-\mathrm{id}\right) \text { or } V \subseteq \operatorname{ker}\left(\phi^{k}+\mathrm{id}\right)\right\} .
$$

(i) Obviously, $\operatorname{ker}\left(\phi^{k}-\right.$ id $)=0$ holds for all $k \in \mathbb{Z}$ if and only if $[\mathcal{V C Y} \backslash \mathcal{F} \mathcal{I N}]_{f} / G$ is empty. In this case, $\operatorname{hdim}^{G}(\underline{\underline{E}} G)=n+1$ because of Theorem 5.13 (i). We are left to treat the case that there exists $n \geq 1$ such that $\operatorname{cd}\left(\operatorname{ker}\left(\phi^{n}-\mathrm{id}\right)\right)=1$, while $\operatorname{cd}\left(\operatorname{ker}\left(\phi^{k}-\mathrm{id}\right)\right) \leq 1$ holds for all $k \geq 1$. Put $V:=\operatorname{ker}\left(\phi^{n}-\mathrm{id}\right)$. Then the the index of $N_{G} V$ in $G$ is finite, and so $[V] \cdot G$ belongs to $[\mathcal{V C Y} \backslash \mathcal{F} \mathcal{I N}]_{f} / G$. Consider another element $[W] \cdot G \in[\mathcal{V C Y} \backslash \mathcal{F} \mathcal{I N}]_{f} / G$. There exists $k \geq 1$ such that $W \subseteq \operatorname{ker}\left(\phi^{k}-\mathrm{id}\right)$ or $W \subseteq \operatorname{ker}\left(\phi^{k}+\mathrm{id}\right)$, hence $W \subseteq \operatorname{ker}\left(\phi^{2 k n}-\mathrm{id}\right)$. Since also $V \subseteq \operatorname{ker}\left(\phi^{2 k n}-\mathrm{id}\right)$ and $\operatorname{cd}\left(\operatorname{ker}\left(\phi^{2 k n}-\mathrm{id}\right)\right) \leq 1$, we conclude $[V] \cdot G=[W] \cdot G$, and we have shown that $[\mathcal{V C Y} \backslash \mathcal{F} \mathcal{I N}]_{f} / G=\{[V] \cdot G\}$ contains precisely one element $[V] \cdot G$.

Now $\operatorname{hdim}^{G}(\underline{\underline{E}} G)=n+1$ follows from Theorem 5.13 (ii)(b). Namely, any infinite cyclic subgroup $C \subseteq \mathbb{Z}^{n}$ such that $C \cap V=\{1\}$ yields an element $[C] \cdot G \in$ $[\mathcal{V C Y} \backslash \mathcal{F} \mathcal{I N}]_{f} / G$ such that $\operatorname{cd}\left(N_{G}[C]\right)=n=\operatorname{cd}(G)-1$.

(ii) Choose $k \geq 1$ with $\operatorname{cd}\left(\operatorname{ker}\left(\phi^{k}-\right.\right.$ id $\left.)\right) \geq 2$ and put $G^{\prime}:=p^{-1}(k \mathbb{Z})$. Then $G^{\prime}$ is a subgroup of $G$ of finite index whose center contains $\mathbb{Z}^{2}$, and hdim ${ }^{G}(\underline{\underline{E}} G)=n+2$ follows from Theorem 5.13 (iii).

Theorem 5.23. Let $1 \rightarrow \mathbb{Z}^{m} \rightarrow G \stackrel{p}{\rightarrow} \mathbb{Z}^{n} \rightarrow 1$ be a group extension such that $\operatorname{cent}(G)=\mathbb{Z}^{m}$. Then

$$
\operatorname{hdim}^{G}(\underline{E} G)=\operatorname{cd}(G)=m+n
$$

and

$$
\operatorname{hdim}^{G}(\underline{\underline{E}} G)= \begin{cases}0 & \text { if } m+n \leq 1 \\ n+1 & \text { if } m=1 \text { and } n \geq 1 \\ m+n+1 & \text { if } m \geq 2 \text { and } n \geq 1\end{cases}
$$

Proof. The group $G$ is poly- $\mathbb{Z}$ by Lemma 5.14 (i). We get $\operatorname{hdim}^{G}(\underline{E} G)=\operatorname{cd}(G)=$ $m+n$ from Theorem 5.13 and Lemma 5.14 (iv).

If $m+n \leq 1$, then $G$ is infinite cyclic, so $\operatorname{hdim}^{G}(\underline{\underline{E}} G)=0$. If $m=0$, then $G=\mathbb{Z}^{n}$ and the claim follows from Example 5.21. If $m \geq 2$, the claim follows 
from Theorem 5.13 (iii). It remains to treat the case $m=1$ and $n \geq 1$, in which we want to show for an infinite cyclic subgroup $C \subseteq G$ with $[C] \neq[\operatorname{cent}(G)]$ that $\operatorname{cd}\left(N_{G}[C]\right)=n$.

Consider the canonical projection $p: G \rightarrow G / \operatorname{cent}(G) \cong \mathbb{Z}^{n}$. According to Lemma 5.15, we can assume that $N_{G} C=N_{G}[C]$. Since $C_{G} C$ has finite index in $N_{G} C$, it suffices to prove that $\operatorname{cd}\left(C_{G} C\right)=n$. Fix a generator $z \in C$ and an element $g \in G$. Let $c(g): G \rightarrow G$ be the automorphism sending $g^{\prime}$ to $g g^{\prime} g^{-1}$, then $p \circ c(g)(z)=p(z)$. There is, therefore, precisely one element $\sigma(g) \in \operatorname{cent}(G)$ satisfying $c(g)(z)=z \sigma(g)$. A straightforward computation for $g_{1}, g_{2} \in G$ shows that $z \sigma\left(g_{1} g_{2}\right)=z \sigma\left(g_{1}\right) \sigma\left(g_{2}\right)$, which implies $\sigma\left(g_{1} g_{2}\right)=\sigma\left(g_{1}\right) \sigma\left(g_{2}\right)$. Moreover, $\sigma(1)=1$. Thus, we have defined a group homomorphism $\sigma: G \rightarrow \operatorname{cent}(G)$ whose kernel is $C_{G} C$. As $[C] \neq[\operatorname{cent}(G)]$ by assumption, $\operatorname{cent}(G)$ cannot contain $C$, so $\operatorname{ker}(\sigma) \neq G$. This means that $\sigma$ is non-trivial and that, hence, its image is an infinite cyclic group. We conclude from Lemma 5.14 (iv) that $\operatorname{cd}\left(C_{G} C\right)=n$, as we wanted to show.

The above implies that we are in the situation of Theorem 5.13 (ii)(b), and we get $\operatorname{hdim}^{G}(\underline{\underline{E}} G)=\operatorname{cd}(G)=n+1$.

Theorem 5.24. Let $1 \rightarrow \mathbb{Z} \rightarrow H \stackrel{p}{\rightarrow} \mathbb{Z}^{n} \rightarrow 1$ be a group extension such that $\mathbb{Z}=\operatorname{cent}(H)$ and $n \geq 2$. Consider a group automorphism $f: H \rightarrow H$. It sends $\operatorname{cent}(H)$ to $\operatorname{cent}(H)$. Let $\bar{f}: \mathbb{Z}^{n} \rightarrow \mathbb{Z}^{n}$ be the group automorphism induced by $f$, i.e., which satisfies $p \circ f=\bar{f} \circ p$. Then $G:=H \rtimes_{f} \mathbb{Z}$ satisfies $\operatorname{cd}(G)=n+2$, we have

$$
\operatorname{hdim}^{G}(\underline{E} G)=n+2,
$$

and precisely one of the following cases occurs:

(i) We have $\operatorname{ker}\left(\bar{f}^{k}-\mathrm{id}\right)=0$ for every $k \in \mathbb{Z}, k \neq 0$. In this case,

$$
\operatorname{hdim}^{G}(\underline{\underline{E}} G)=n+1
$$

(ii) We have $\operatorname{ker}\left(\bar{f}^{k}-\mathrm{id}\right) \neq 0$ for some $k \in \mathbb{Z}, k \neq 0$ and $\bar{f}$ is not periodic, i.e., there is no $l \in \mathbb{Z}, l \geq 1$ such that $\bar{f}^{l}=\mathrm{id}$. In this case,

$$
\operatorname{hdim}^{G}(\underline{\underline{E}} G)=n+2
$$


(iii) The map $\bar{f}$ is periodic. In this case,

$$
\operatorname{hdim}^{G}(\underline{\underline{E}} G)=n+3
$$

Proof. Let pr: $G=H \rtimes_{f} \mathbb{Z} \rightarrow \mathbb{Z}$ be the obvious projection, then $G$ fits into an exact sequence $1 \rightarrow H \rightarrow G \stackrel{\mathrm{pr}}{\longrightarrow} \mathbb{Z} \rightarrow 1$. Therefore, $\operatorname{hdim}^{G}(\underline{E} G)=\operatorname{cd}(G)=n+2$ follows from Lemma 5.14 (i) and (iv) and Theorem 5.13.

Let $V \subseteq G$ be an infinite cyclic subgroup. Then $V \subseteq \operatorname{cent}(H)$ if and only if $[V]=[\operatorname{cent}(H)]$ and, in this case, $N_{G}[V]=G$. Provided that $[V] \neq[\operatorname{cent}(H)]$, we first want to show

$$
\operatorname{cd}\left(N_{G}[V]\right)=\left\{\begin{array}{lr}
n & \text { if } \operatorname{pr}\left(N_{G}[V]\right)=0 \\
n+1 & \text { if } \operatorname{pr}\left(N_{G}[V]\right) \neq 0 \\
& \text { and } \operatorname{pr}(V)=0 \\
2+\operatorname{cd}\left(p\left(N_{G}[V] \cap H\right)\right) & \text { if } \operatorname{pr}(V) \neq 0 .
\end{array}\right.
$$

Suppose that $\operatorname{pr}(V)=0$, i.e., $V \subseteq H$. We have already seen in the proof of Theorem 5.23 that $\operatorname{cd}\left(N_{H}[V]\right)=n$ for $[V] \neq[\operatorname{cent}(H)]$. It follows from the short exact sequence

$$
1 \rightarrow N_{H}[V]=N_{G}[V] \cap H \rightarrow N_{G}[V] \rightarrow \operatorname{pr}\left(N_{G}[V]\right) \rightarrow 1
$$

and Lemma 5.14 (iv) that $\operatorname{cd}\left(N_{G}[V]\right)=n+\operatorname{cd}\left(\operatorname{pr}\left(N_{G}[V]\right)\right)$. If $\operatorname{pr}\left(N_{G}[V]\right)=0$, then $\operatorname{cd}\left(\operatorname{pr}\left(N_{G}[V]\right)\right)=0$. If $\operatorname{pr}\left(N_{G}[V]\right) \neq 0$, then $\operatorname{pr}\left(N_{G}[V]\right) \cong \mathbb{Z}$, which implies $\operatorname{cd}\left(\operatorname{pr}\left(N_{G}[V]\right)\right)=1$.

Now suppose that $\operatorname{pr}(V) \neq 0$. Then $\operatorname{pr}(V)$ is infinite cyclic, so the same must be true for $\operatorname{pr}\left(N_{G}[V]\right)$. Let $w$ be a generator of $\operatorname{cent}(H)$ and $v$ be a generator of $V$. Since $f$ induces \pm id on $\operatorname{cent}(H)$, we have $v w v^{-1}=w^{ \pm 1}$. This implies $v w^{2} v^{-1}=w^{2}$, thus $w^{2} \in C_{G} V \subseteq N_{G}[V]$. Therefore, $N_{G}[V] \cap \operatorname{cent}(H)$ is an infinite cyclic group, and the short exact sequences

$$
1 \rightarrow N_{G}[V] \cap \operatorname{cent}(H) \rightarrow N_{G}[V] \cap H \rightarrow p\left(N_{G}[V] \cap H\right) \rightarrow 1
$$

and (5.26) together with Lemma 5.14 (iv) imply $\operatorname{cd}\left(N_{G}[V]\right)=2+\operatorname{cd}\left(p\left(N_{G}[V] \cap\right.\right.$ $H)$ ). This finishes the proof of $(5.25)$. 
Next, we show in the case $\operatorname{pr}(V) \neq 0$ that

$$
p\left(N_{G}[V] \cap H\right) \subseteq \operatorname{ker}\left(\bar{f}^{k}-\mathrm{id}\right) \quad \text { for some } k \in \mathbb{Z}, k \neq 0 .
$$

Because of Lemma 5.15, we can assume without loss of generality that $N_{G}[V]=$ $N_{G} V$. Recall that $C_{G} V$ has finite index in $N_{G} V$. Hence it suffices to show that $p\left(C_{G} V \cap H\right) \subseteq \operatorname{ker}\left(\bar{f}^{k}-\right.$ id $)$ if $k \in \operatorname{pr}(V)$. Let $t \in G=H \rtimes_{f} \mathbb{Z}$ be given by the generator of $\mathbb{Z}$. Then conjugation with $t$ induces the automorphism $f$ on $H$, and $h t^{k} \in V$ for some $h \in H$. We get for $u \in C_{G} V \cap H$

$$
\begin{aligned}
\bar{f}^{k}(p(u))=p \circ f^{k}(u)=p\left(t^{k} u t^{-k}\right)=p(h) p\left(t^{k} u t^{-k}\right) p(h)^{-1} & \\
& =p\left(h t^{k} u\left(h t^{k}\right)^{-1}\right)=p(u),
\end{aligned}
$$

which finishes the proof of (5.27).

Finally, we show in the case $\operatorname{pr}\left(N_{G}[V]\right) \neq 0$ and $\operatorname{pr}(V)=0$ that

$$
p(V) \subseteq \operatorname{ker}\left(\bar{f}^{k}-\mathrm{id}\right) \quad \text { for some } k \in \mathbb{Z}, k \neq 0 .
$$

Again, we can assume that $N_{G}[V]=N_{G} V$. Since $C_{G} V$ has finite index in $N_{G} V$, we have $\operatorname{pr}\left(C_{G}[V]\right) \neq 0$, so we can choose a non-trivial element $k \in \operatorname{pr}\left(C_{G}[V]\right)$. Then $h t^{k} \in C_{G} V$ for some $h \in H$, and the same calculation as above shows that $\bar{f}^{k}(p(v))=p(v)$ holds for $v \in V$. This finishes the proof of (5.28). Now we are ready to prove the assertions appearing in Theorem 5.24.

(i) Consider an infinite cyclic subgroup $V \subseteq G$ such that $[V] \neq[\operatorname{cent}(H)]$. We first deal with the case $\operatorname{pr}(V)=0$. Then $\operatorname{pr}\left(N_{G}[V]\right)=0$ since, otherwise, (5.28) would yield $V \subseteq \operatorname{cent}(H)$. Using (5.25), the conclusion is that $\operatorname{cd}\left(N_{G}[V]\right)=n$. Since $\operatorname{cd}(G)=n+2$, we get hdim ${ }^{G}(\underline{\underline{E}} G)=n+1$ from Theorem 5.13 (ii)(a). It remains to consider the case $\operatorname{pr}(V) \neq 0$. Then we conclude $\operatorname{cd}\left(N_{G}[V]\right)=2+\operatorname{cd}\left(p\left(N_{G} V \cap H\right)\right)$ from (5.25). Because of (5.27) we get $\operatorname{cd}\left(N_{G}[V]\right)=2$. Since $n \geq 2$, we have $\operatorname{vcd}(G) \geq 4$. Now $\operatorname{hdim}^{G}(\underline{\underline{E}} G)=n+1$ follows from Theorem 5.13 (ii)(a).

(ii) We claim that there exists an infinite cyclic subgroup $V \subseteq H$ such that $[V] \neq[\operatorname{cent}(H)], \operatorname{pr}(V)=0$ and $\operatorname{pr}\left(N_{G} V\right) \neq 0$. To do so, we choose an even $k \in \mathbb{Z}$ such that $\operatorname{ker}\left(\bar{f}^{k}-\mathrm{id}\right) \neq 0$, an element $h_{0} \in H$ such that $0 \neq p\left(h_{0}\right) \in \operatorname{ker}\left(\bar{f}^{k}-\mathrm{id}\right)$, and define $V$ to be the infinite cyclic group generated by $h_{0}$. Then $p\left(f^{k}\left(h_{0}\right)\right)=$ $\bar{f}^{k} \circ p\left(h_{0}\right)=p\left(h_{0}\right)$. Hence, we can find $z_{0} \in \operatorname{cent}(H)=\mathbb{Z}$ such that $t^{k} h_{0} t^{-k}=$ 
$f^{k}\left(h_{0}\right)=h_{0} z_{0}$. Since $k$ is even, $t^{k} z_{0} t^{-k}=z_{0}$. As $h_{0} \notin \operatorname{cent}(H)$, we can find $h_{1} \in$ $H$ such that $h_{1} h_{0} h_{1}^{-1} \neq h_{0}$. However, $p\left(h_{1} h_{0} h_{1}^{-1}\right)=p\left(h_{1}\right) p\left(h_{0}\right) p\left(h_{1}^{-1}\right)=p\left(h_{0}\right)$ and so $h_{1} h_{0} h_{1}^{-1}=h_{0} z_{1}$ for some non-trivial $z_{1} \in \operatorname{cent}(H)$. Choose integers $m_{0} \neq 0$ and $m_{1}$ such that $z_{0}^{m_{0}}=z_{1}^{m_{1}}$, and put $u=h_{1}^{-m_{1}} t^{m_{0} k}$. From $t^{m_{0} k} h_{0} t^{-m_{0} k}=h_{0} z_{0}^{m_{0}}$ and $h_{1}^{-m_{1}} h_{0} h_{1}^{m_{1}}=h_{0} z_{1}^{-m_{1}}$ we then conclude $u h_{0} u^{-1}=h_{0}$ and $\operatorname{pr}(u)=k m_{0}$. This finishes the proof of the above claim. It follows from (5.25) that $\operatorname{cd}\left(N_{G}[V]\right)=$ $n+1$.

Since $\operatorname{cd}\left(\operatorname{ker}\left(\bar{f}^{k}-\mathrm{id}\right)\right)=n$ implies $\bar{f}^{k}=\mathrm{id}$ but $\bar{f}$ is not periodic, we have $\operatorname{cd}\left(\operatorname{ker}\left(\bar{f}^{k}-\mathrm{id}\right)\right) \leq n-1$ for every $k \in \mathbb{Z}$ with $k \neq 0$. Thus, (5.25) and (5.27) show that $\operatorname{cd}\left(N_{G}[V]\right) \leq n+1$ holds for all infinite cyclic subgroups $V \subseteq G$ satisfying $[V] \neq[\operatorname{cent}(H)]$. Theorem 5.13 (ii)(b) now implies $\operatorname{hdim}^{G}(\underline{\underline{E}} G)=n+2$.

(iii) We want to show the existence of a finite index subgroup of $G$ whose center contains $\mathbb{Z}^{2}$. Then $\operatorname{hdim}^{G}(\underline{\underline{E}} G)=n+3$ follows from Theorem 5.13 (iii). Let us begin by choosing an even $k \in \mathbb{Z}$ such that $\bar{f}^{k}=$ id. We define a group homomorphism $\sigma_{f^{k}}: H \rightarrow \operatorname{cent}(H)=\mathbb{Z}$ by $\sigma_{f^{k}}(h):=h^{-1} f^{k}(h)$ for $h \in H$. Since $k$ is even, the restriction of $f^{k}$ to $\operatorname{cent}(H)=\mathbb{Z}$ is the identity, which implies that the restriction of $\sigma_{f^{k}}$ to $\operatorname{cent}(H)$ is trivial. Hence $\sigma_{f^{k}}$ factorizes through the projection $p: H \rightarrow H / \operatorname{cent}(H)=\mathbb{Z}^{n}$, yielding homomorphism $\bar{\sigma}_{f^{k}}: \mathbb{Z}^{n} \rightarrow \mathbb{Z}$. We have $f^{k}(h)=h \bar{\sigma}_{f^{k}}(p(h))$ for $h \in H$.

Conjugation with $h_{0} \in H$ induces a group automorphism $c\left(h_{0}\right): H \rightarrow H$ whose restriction to $\operatorname{cent}(H)$ is the identity and for which the induced automorphism $\overline{c\left(h_{0}\right)}$ of $H / \operatorname{cent}(H)=\mathbb{Z}^{n}$ is the identity. Just as above for $f^{k}$, we define a homomorphism $\bar{\sigma}_{c\left(h_{0}\right)}: \mathbb{Z}^{n} \rightarrow \mathbb{Z}$ such that $c\left(h_{0}\right)(h)=h_{0} h h_{0}^{-1}=h \bar{\sigma}_{c\left(h_{0}\right)}(p(h))$ holds for $h \in H$, and one easily checks that

$$
\bar{\sigma}_{c\left(h_{0} h_{1}\right)}=\bar{\sigma}_{c\left(h_{0}\right) \circ c\left(h_{1}\right)}=\bar{\sigma}_{c\left(h_{0}\right)}+\bar{\sigma}_{c\left(h_{0}\right)}
$$

for $h_{0}, h_{1} \in H$. That way, we obtain an injective group homomorphism

$$
\bar{\sigma}: H / \operatorname{cent}(H) \rightarrow \operatorname{hom}_{\mathbb{Z}}(H / \operatorname{cent}(H), \mathbb{Z}), \quad \bar{h} \mapsto \bar{\sigma}_{c(h)} .
$$

Since its source and target are isomorphic to $\mathbb{Z}^{n}$, its image has finite index. As $\bar{\sigma}_{f^{l k}}=l \cdot \bar{\sigma}_{f^{k}}$ holds for all $l \in \mathbb{Z}$, it follows that we can find an integer $l \geq 1$ and $h \in H$ such that $\bar{\sigma}_{f l k}=\bar{\sigma}_{c(h)}$, which implies $f^{k l}=c(h)$. 
The subgroup $\operatorname{pr}^{-1}(k l \cdot \mathbb{Z}) \subseteq G$ has finite index in $G$ and is isomorphic to the semidirect product $H \rtimes_{f k l} \mathbb{Z}$ with respect to the automorphism $f^{k l}: H \rightarrow H$. Since $f^{k l}$ is an inner automorphism, $\mathrm{pr}^{-1}(k l \cdot \mathbb{Z})$ is isomorphic to the direct product $H \times \mathbb{Z}$ and, hence, contains $\operatorname{cent}(H) \times \mathbb{Z} \cong \mathbb{Z}^{2}$ in its center. This finishes the proof of Theorem 5.24.

Example 5.29 (Three-dimensional Heisenberg group). The three-dimensional Heisenberg group is the group given by the presentation Hei $=\langle u, v, z|[u, v]=$ $z,[u, z]=1,[v, z]=1\rangle$. Define automorphisms $f_{k}$ : Hei $\rightarrow$ Hei for $k=-1,0,1$ by

$$
\begin{aligned}
& f_{-1}(u)=u^{3} v, \quad f_{-1}(v)=u^{2} v, \quad f_{-1}(z)=z ; \\
& f_{0}(u)=u, \quad f_{0}(v)=u v, \quad f_{0}(z)=z ; \\
& f_{1}(u)=u, \quad f_{1}(v)=v, \quad f_{1}(z)=z .
\end{aligned}
$$

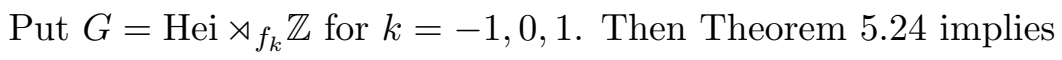

$$
\begin{aligned}
\operatorname{hdim}^{G_{k}}\left(\underline{E} G_{k}\right) & =4 ; \\
\operatorname{hdim}^{G_{k}}\left(\underline{\underline{E}} G_{k}\right) & =4+k .
\end{aligned}
$$

\subsection{Models for $E_{\mathcal{S F G}}(G)$}

Let $\mathcal{S F \mathcal { G }}$ be the family of subgroups of $G$ which are contained in some finitely generated subgroup, i.e., $H \in \mathcal{S F \mathcal { G }}$ if and only if there exists a finitely generated subgroup $H^{\prime} \subseteq G$ such that $H \subseteq H^{\prime}$. This is the smallest family of subgroups of $G$ which contains all finitely generated subgroups of $G$.

Example 5.30. Suppose that $G$ is countable. Then there exists a one-dimensional model for $E_{\mathcal{S F G}}(G)$ because of Theorem 4.3 and Lemma 4.2. In particular, there exists a one-dimensional model for $\underline{E} G$ or $\underline{\underline{E}} G$ if $G$ is locally finite or locally virtually cyclic, respectively.

Theorem 5.31. Let $G$ be an infinite group of cardinality $\aleph_{n}$. Let $\mathcal{S F G}$ be the family of subgroups defined above. Then

$$
\operatorname{hdim}^{G}\left(E_{\mathcal{S F G}}(G)\right) \leq n+1
$$


Proof. We use induction on $n \in \mathbb{N}$. The case $n=0$ has already been settled in Example 5.30. The induction step from $n-1$ to $n \geq 1$ is done as follows.

We can write $G=\bigcup_{\alpha<\omega_{n}} G_{\alpha}$ for subgroups $G_{\alpha}$ of cardinality $\aleph_{n-1}$ such that $G_{\alpha} \subseteq G_{\beta}$ if $\alpha \leq \beta$. By induction hypothesis, for $\alpha<\omega_{n}$ there are models $X_{\alpha}$ for $E_{\mathcal{S} \mathcal{F} \mathcal{G}}\left(G_{\alpha}\right)$ of dimension $n$. The induction step now must provide us with an $(n+1)$-dimensional model for $E_{\mathcal{S} \mathcal{F}}(G)$. If we set $G_{\omega_{n}}:=G$, this will be accomplished by using transfinite induction for $\alpha \leq \omega_{n}$ to construct $(n+1)$ dimensional models $Y_{\alpha}$ for $E_{\mathcal{S F \mathcal { G }}}\left(G_{\alpha}\right)$ such that $G_{\gamma} \times_{G_{\alpha}} Y_{\alpha} \subseteq G_{\gamma} \times{ }_{G_{\beta}} Y_{\beta}$ is a

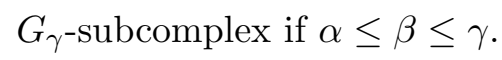

Let $Y_{0}:=X_{0}$. Now suppose that $\alpha$ has got a predecessor. Then the universal property of $E_{\mathcal{S} \mathcal{F} \mathcal{G}}\left(G_{\alpha}\right)$ yields a $G_{\alpha}$-map $f_{\alpha}: G_{\alpha} \times_{G_{\alpha-1}} X_{\alpha-1} \rightarrow X_{\alpha}$, which we can assume to be cellular by the equivariant cellular approximation theorem. We define $Y_{\alpha}$ by the $G_{\alpha}$-pushout

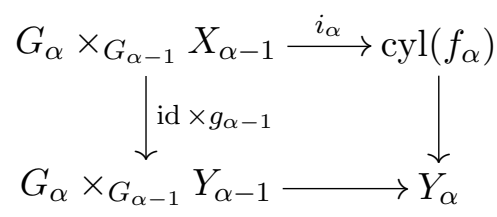

in which $i_{\alpha}$ is the obvious inclusion into the mapping cylinder of $f_{\alpha}$ and $g_{\alpha-1}$ the up to $G_{\alpha-1}$-homotopy unique homotopy equivalence which comes from the universal property of $E_{\mathcal{S F \mathcal { G }}}\left(G_{\alpha-1}\right)$. Hence, $Y_{\alpha}$ is $G_{\alpha}$-homotopy equivalent to $X_{\alpha}$ and therefore a model for $E_{\mathcal{S} \mathcal{F} \mathcal{G}}\left(G_{\alpha}\right)$. Moreover, $Y_{\alpha}$ is clearly $(n+1)$-dimensional. Finally, if $\alpha$ is a limit ordinal, we define $Y_{\alpha}$ to be the union of the $G_{\alpha} \times{ }_{G_{\beta}} Y_{\beta}$ for $\beta<\alpha$.

Example 5.32. Let $G$ be an infinite locally finite group of cardinality $\aleph_{n}$. Then it is consistent with ZFC (Zermelo-Fraenkel plus axiom of choice) that

$$
\operatorname{hdim}^{G}(\underline{E} G)=n+1
$$

The existence of an $(n+1)$-dimensional model for $\underline{E} G$ follows from Theorem 5.31 and has already been proved by Dicks-Kropholler-Leary [8, Theorem 2.6]. Now the claim follows from the fact that it is consistent with ZFC that the cohomological dimension over $\mathbb{Q}$ of $G$ satisfies $\operatorname{cd}_{\mathbb{Q}}(G)=n+1$ (see [12, Theorem A]). 
We emphasize that the assumption in Example 5.30 that $G$ is countable is necessary. Namely, Dunwoody [9, Theorem 1.1] has proved that for a group $K$ there is a one-dimensional model for $\underline{E} K$ if and only if $\operatorname{cd}_{\mathbb{Q}}(K) \leq 1$.

\subsection{Low dimensions}

For countable groups $G$, the question whether there are models for $\underline{E} G$ and $\underline{\underline{E}} G$ of small dimension has the following answer. The assumption that $G$ is countable is essential (see Example 5.32).

Theorem 5.33. (i) Let $G$ be a countable group which is locally virtually cyclic.

Then

$$
\operatorname{hdim}^{G}(\underline{E} G)= \begin{cases}0 & \text { if } G \text { is finite; } \\ 1 & \text { if } G \text { is infinite and either locally finite } \\ 2 & \text { otherwise, }\end{cases}
$$

and

$$
\operatorname{hdim}^{G}(\underline{\underline{E}} G)= \begin{cases}0 & \text { if } G \text { is virtually cyclic } \\ 1 & \text { otherwise }\end{cases}
$$

(ii) Let $G$ be a countable group satisfying $\operatorname{hdim}^{G}(\underline{E} G) \leq 1$. Then

$$
\operatorname{hdim}^{G}(\underline{\underline{E}} G)= \begin{cases}0 & \text { if } G \text { is virtually cyclic; } \\ 1 & \text { if } G \text { is locally virtually cyclic but } \\ 2 & \text { otherwiser } r\end{cases}
$$

Proof. (i) The claim about hdim ${ }^{G}(\underline{\underline{E}} G)$ follows from Example 5.30.

We conclude hdim${ }^{G}(\underline{E} G) \leq 2$ from $\operatorname{hdim}^{G}(\underline{\underline{E}} G) \leq 1$ and Corollary 5.4 (i). If $G$ is locally finite, then $\operatorname{hdim}^{G}(\underline{E} G) \leq 1$ has already been proved in Example 5.30. Let $G$ be a countable locally virtually cyclic group and $\operatorname{hdim}^{G}(\underline{E} G) \leq 1$. It remains to show that $G$ is virtually cyclic or locally finite. Assume that $G$ is not 
locally finite, then we can choose choose a sequence of infinite virtually cyclic subgroups $V_{0} \subseteq V_{1} \subseteq V_{2} \subseteq \ldots$ such that $G=\bigcup_{i \geq 0} V_{i}$, and we must show that $G$ itself is virtually cyclic.

We begin with the case that each $V_{i}$ is an infinite virtually cyclic group of type I. Type I means that we can find an epimorphism $p_{i}: V_{i} \rightarrow C_{i}$ onto an infinite cyclic group $C_{i}$. Let $F_{i}$ be the kernel of $p_{i}$, which is a finite group. Thus we obtain a nested sequence of inclusions of finite groups $F_{1} \subseteq F_{2} \subseteq \ldots$ and a sequence of inclusions of infinite cyclic groups $C_{1} \subseteq C_{2} \subseteq \ldots$ such that there exists a short exact sequence $1 \rightarrow F_{i} \rightarrow V_{i} \rightarrow C_{i} \rightarrow 1$ for each $i$.

Now we deal with the hardest step in the proof, where we show that the sequence $F_{0} \subseteq F_{1} \subseteq F_{2} \subseteq \ldots$ is eventually stationary. Let $t \in V_{0}$ be an element which is mapped to a generator under the epimorphism $p_{0}: V_{0} \rightarrow C_{0}$. If we consider $t$ as an element in $V_{i}$, conjugation with $t$ induces an automorphism $\phi_{i}: F_{i} \rightarrow F_{i}$. Obviously, the restriction of $\phi_{i}$ to $F_{i-1}$ is $\phi_{i-1}$. We obtain a nested sequence of virtually cyclic groups $F_{0} \rtimes \mathbb{Z} \subseteq F_{1} \rtimes \mathbb{Z} \subseteq F_{2} \rtimes \mathbb{Z} \subseteq \ldots$, where the $i$-th semi-direct product $F_{i} \rtimes \mathbb{Z}$ is to be understood with respect to $\phi_{i}$. Since $K:=\bigcup_{i \geq 0} F_{i} \rtimes \mathbb{Z}$ is a subgroup of $G$, it satisfies $\operatorname{hdim}^{K}(\underline{E} K) \leq 1$. This implies $H_{K}^{2}(\underline{E} K ; M)=0$ for any $\mathbb{Q}[K]$-module $M$.

The $\mathbb{Q}[K]$-chain map $C_{*}(E K) \rightarrow C_{*}(\underline{E} K)$ of the cellular $\mathbb{Q}[K]$-chain complexes coming from a $K$-map $E K \rightarrow \underline{E} K$ induces an isomorphism on homology. Since both of these $\mathbb{Q}[K]$-chain complexes are projective, this map is a $\mathbb{Q}[K]$ chain homotopy equivalence. Hence, we obtain for every $\mathbb{Q}[K]$-module $M$ an isomorphism

$$
\begin{array}{r}
H^{k}(K ; M)=H^{k}\left(\operatorname{hom}_{\mathbb{Q}[K]}\left(C_{*}(E K), M\right)\right) \cong H^{k}\left(\operatorname{hom}_{\mathbb{Q}[K]}\left(C_{*}(\underline{E} K), M\right)\right) \\
=H_{K}^{k}(\underline{E} K ; M) .
\end{array}
$$

In particular, $H^{2}(K ; M)=0$.

If we put $F=\bigcup_{i \geq 0} F_{i}$, then the collection of the $\phi_{i}$-s yields an automorphism $\phi: F \rightarrow F$, with respect to which $K=F \rtimes \mathbb{Z}$. Let $M$ be any $\mathbb{Q}[K]$-module. The Hochschild-Serre spectral sequence associated to $1 \rightarrow F \rightarrow K=F \rtimes \mathbb{Z} \rightarrow \mathbb{Z} \rightarrow 1$ yields the exact sequence

$$
H^{1}(F ; M) \stackrel{\text { id }-\phi_{*}}{\longrightarrow} H^{1}(F ; M) \rightarrow H^{2}(K ; M) \rightarrow H^{2}(F ; M)
$$


where the automorphism $\phi_{*}$ of $H^{1}(F ; M)$ is the one induced by $\phi: F \rightarrow F$ and the $\mathbb{Q}[F \rtimes \mathbb{Z}]$-structure on $M$. Hence

$$
H^{2}(K ; M) \cong \operatorname{coker}\left(\mathrm{id}-\phi_{*}: H^{1}(F ; M) \rightarrow H^{1}(F ; M)\right)
$$

since we will show in a moment that $H^{2}(F ; M)=0$.

Next, we compute $H^{*}(F ; M)$ for a given $\mathbb{Q}[F]$-module $M$. We have already explained the isomorphism

$$
H^{1}(F ; M)=H^{1}\left(\operatorname{hom}_{\mathbb{Q}[F]}\left(C_{*}(E F), M\right)\right) \cong H^{1}\left(\operatorname{hom}_{\mathbb{Q}[F]}\left(C_{*}(\underline{E} F), M\right)\right) .
$$

It follows from Lemma 4.5 that we obtain an $F$-pushout

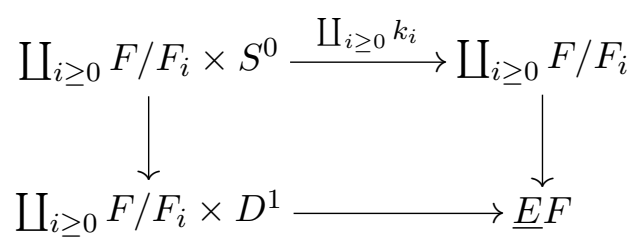

where the left vertical arrow is the canonical inclusion and $k_{i}: F / F_{i} \times S^{0} \rightarrow$ $\bigsqcup_{i \geq 0} F / F_{i}$ sends $(u,-1)$ to $u \in F / F_{i}$ and $(u, 1)$ to the image of $u$ under the projection $F / F_{i} \rightarrow F / F_{i+1}$. Hence, the cellular $\mathbb{Q}[F]$-chain complex of $\underline{E} F$ is concentrated in dimensions 0 and 1 and is given there by

$$
\bigoplus_{i \geq 0} \mathbb{Q}\left[F / F_{i}\right] \stackrel{\oplus_{i \geq 0} l_{i}}{\longrightarrow} \bigoplus_{i \geq 0} \mathbb{Q}\left[F / F_{i}\right],
$$

where $l_{i}$ sends $u \in \mathbb{Q}\left[F / F_{i}\right]$ to the difference of the element given by $u$ itself and the element given by the image of $u$ under $\mathbb{Q}\left[\mathrm{pr}_{i}\right]: \mathbb{Q}\left[F / F_{i}\right] \rightarrow \mathbb{Q}\left[F / F_{i+1}\right]$ for $\operatorname{pr}_{i}: F / F_{i} \rightarrow F / F_{i+1}$ the canonical projection. Therefore, $H^{k}(F ; M)=0$ for $k \geq 2$, whereas $H^{1}(F ; M)$ is the cokernel of the $\mathbb{Q}[F]$-homomorphism

$$
\delta_{M}: \prod_{i \geq 0} M^{F_{i}} \rightarrow \prod_{i \geq 0} M^{F_{i}}, \quad\left(x_{i}\right)_{i \geq 0} \mapsto\left(x_{i}-x_{i+1}\right)_{i \geq 0} .
$$

Let us show that $H^{1}(F ; \mathbb{Q}[F])=0$ if and only if the sequence $F_{0} \subseteq F_{1} \subseteq$ $F_{2} \subseteq \ldots$ is eventually stationary. If it becomes stationary, then $F$ is finite, so $H^{1}(F ; \mathbb{Q}[F])=0$. Suppose that $H^{1}(F ; \mathbb{Q}[F])=0$ and let $N_{F_{i}} \in \mathbb{Q}[F]^{F_{i}}$ be 
the element $\sum_{f \in F_{i}} f$. Since $\delta_{\mathbb{Q}[F]}$ is surjective, there exists $\left(x_{i}\right)_{i \geq 0} \in \prod_{i \geq 0} \mathbb{Q}[F]^{F_{i}}$ satisfying $N_{F_{i}}=x_{i}-x_{i+1}$. Thus, we get for $i \geq 1$

$$
x_{i}=x_{0}-N_{G_{i-1}}-N_{G_{i-2}}-\cdots-N_{G_{0}} .
$$

After choosing $i_{0}$ in such a way that $x_{0} \in \mathbb{Q}\left[F_{i_{0}}\right]$, we get that $x_{i} \in \mathbb{Q}\left[F_{i-1}\right]$ holds for for $i>i_{0}$. Since $x_{i} \in \mathbb{Q}[F]^{F_{i}}$, we conclude that $F_{i}=F_{i-1}$ holds for $i>i_{0}$, which means that $F_{0} \subseteq F_{1} \subseteq F_{2} \subseteq \ldots$ is eventually stationary. For more information about $H^{*}(F ; \mathbb{Q}[F])$ for locally finite groups $F$, we refer for instance to $[8]$.

Now suppose that $M$ is a $\mathbb{Q}[K]$-module. We obtain the commutative diagram

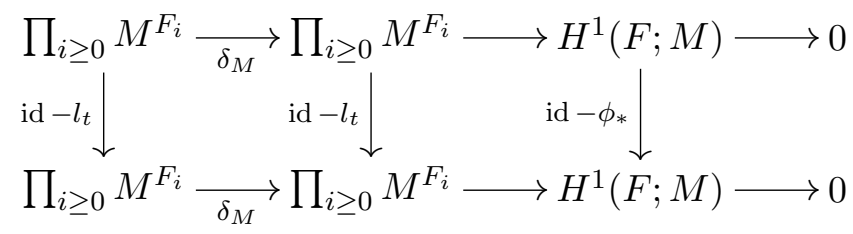

where $l_{t}: M \rightarrow M$ is multiplication with the generator of $\mathbb{Z}$ considered as an element in $K=F \rtimes \mathbb{Z}$.

Specializing to $M=\mathbb{Q}[K]=\mathbb{Q}[F \rtimes \mathbb{Z}]$, we can write down the following exact sequence

$$
0 \rightarrow \mathbb{Q}[F \rtimes \mathbb{Z}] \stackrel{\text { id }-l_{t}}{\longrightarrow} \mathbb{Q}[F \rtimes \mathbb{Z}] \stackrel{\epsilon}{\rightarrow} \mathbb{Q}[F] \rightarrow 0,
$$

where $\epsilon$ sends $\sum_{n \in \mathbb{Z}} r_{i} \cdot f_{i} t^{i}$ to $\sum_{n \in \mathbb{Z}} r_{i} \cdot \phi^{-i}\left(f_{i}\right)$ for $r_{i} \in \mathbb{Q}, f_{i} \in F$ and $t \in \mathbb{Z}$ a fixed generator. The composition

$$
\prod_{i \geq 0} \mathbb{Q}[F \rtimes \mathbb{Z}]^{F_{i}} \stackrel{\delta_{\mathbb{Q}[F \rtimes \mathbb{Z}]}^{\longrightarrow}}{\longrightarrow} \prod_{i \geq 0} \mathbb{Q}[F \rtimes \mathbb{Z}]^{F_{i}} \stackrel{\epsilon}{\rightarrow} \prod_{i \geq 0} \mathbb{Q}[F]^{F_{i}}
$$

agrees with the composition

$$
\prod_{i \geq 0} \mathbb{Q}[F \rtimes \mathbb{Z}]^{F_{i}} \stackrel{\epsilon}{\rightarrow} \prod_{i \geq 0} \mathbb{Q}[F]^{F_{i}} \stackrel{\delta_{\mathbb{Q}[F]}}{\rightarrow} \prod_{i \geq 0} \mathbb{Q}[F]^{F_{i}}
$$

since $\epsilon \circ l_{t}=\epsilon$. Hence we obtain an isomorphism

$$
\begin{aligned}
& H^{1}(F ; \mathbb{Q}[F]) \cong \operatorname{coker}\left(\delta_{\mathbb{Q}[F]}: H^{1}(F ; \mathbb{Q}[F]) \rightarrow H^{1}(F ; \mathbb{Q}[F])\right) \\
& \quad \cong \operatorname{coker}\left(\operatorname{id}-\phi_{*}: H^{1}(F ; \mathbb{Q}[F \rtimes \mathbb{Z}]) \rightarrow H^{1}(F ; \mathbb{Q}[F \rtimes \mathbb{Z}])\right) \cong H^{2}(K ; \mathbb{Q}[K])
\end{aligned}
$$


As $H^{2}(K ; \mathbb{Q}[K])$ is trivial, the same holds for $H^{1}(F ; \mathbb{Q}[F])$. We have already shown that this implies that $F=\bigcup_{i \geq 0} F_{i}$ is finite.

Returning to the beginning of the proof and denoting by $A$ the colimit of the system $C_{0} \subseteq C_{1} \subseteq C_{2} \subseteq \ldots$ of infinite cyclic groups, we obtain the exact sequence $1 \rightarrow F \rightarrow G \rightarrow A \rightarrow 1$. Since $\operatorname{hdim}^{G}(\underline{E} G) \leq 1$ by assumption, the cohomological dimension of $G$ over $\mathbb{Q}$ satisfies $\operatorname{cd}_{\mathbb{Q}}(G) \leq 1$. Consequently, also $\operatorname{cd}_{\mathbb{Q}}(A) \leq 1$ as $F$ is finite. By a result of Dunwoody [9, Theorem 1.1] already mentioned in Example 5.32, this means that $\operatorname{hdim}^{A}(\underline{E} A) \leq 1$. However, as $A$ is torsionfree, this implies that $A$ is free (see [18] and [19]). Since $\mathbb{Z}$ is the only non-trivial abelian free group, $A$ is infinite cyclic, so $G$ is virtually cyclic. This finishes the proof in the case that each $V_{i}$ is infinite cyclic of type I.

We are left to treat the case that there exists $i_{0}$ such that $V_{i_{0}}$ is infinite virtually cyclic of type II, i.e., there exists an epimorphism $V_{i_{0}} \rightarrow D_{\infty}$ onto the infinite dihedral group. Since $V_{i_{0}}$ is a subgroup of $V_{i}$ for $i \geq i_{0}$, the virtually cyclic group $V_{i}$ is of type II for $i \geq i_{0}$ or, equivalently, $H_{1}\left(V_{i}\right)$ is finite for $i \geq i_{0}$. Let $W_{i}$ be the commutator of $V_{i}$. Then we obtain a sequence of virtually cyclic subgroups $W_{0} \subseteq W_{1} \subseteq W_{2} \subseteq \ldots$ such that $W_{i}$ is of type I and has finite index in $V_{i}$ for $i \geq i_{0}$. Let $W$ be the subgroup $\bigcup_{i \geq 0} W_{i}$ of $G$. Since $\operatorname{dim}^{G}(\underline{E} G) \leq 1$ by assumption, we have $\operatorname{hdim}^{W}(\underline{E} W) \leq 1$ as well. Thus, by what we have already proved above, $W$ is virtually cyclic and, in particular, finitely generated. Hence, the sequence $W_{0} \subseteq W_{1} \subseteq W_{2} \subseteq \ldots$ is eventually stationary, and we can assume without loss of generality that $W=W_{i}$ for all $i \geq 0$. This implies that each map $H_{1}\left(V_{i}\right) \rightarrow H_{1}\left(V_{i+1}\right)$ is injective. Putting $A:=\bigcup_{i \geq 0} H_{1}\left(V_{i}\right)$, we obtain an exact sequence $1 \rightarrow W \rightarrow G \rightarrow A \rightarrow 0$, where $W$ is an infinite virtually cyclic group of type I. We are going to show that $A$ is finite.

Let $K$ denote the kernel of the canonical epimorphism $W \rightarrow C$ for $C=$ $H_{1}(W) / \operatorname{tors}\left(H_{1}(W)\right)$. Then $K$ is a finite characteristic subgroup of $W$ and hence normal in $G$, so there is an exact sequence $1 \rightarrow K \rightarrow G \rightarrow G / K \rightarrow$ 1. Note that we have already explained above (using [9, Theorem 1.1]) why $\operatorname{hdim}^{G}(\underline{E} G) \leq 1$ implies hdim ${ }^{G / K}(\underline{E}(G / K)) \leq 1$. Furthermore, there is an exact sequence $1 \rightarrow C \rightarrow G / K \rightarrow A \rightarrow 1$. Let $A \rightarrow$ aut $(C)$ be the associated conjugation homomorphism, let $A^{\prime} \subseteq A$ be its kernel, and let $G^{\prime}$ the preimage of $A^{\prime}$ under the epimorphism $G / K \rightarrow A$. Then we obtain a central extension 
$1 \rightarrow C \rightarrow G^{\prime} \rightarrow A^{\prime} \rightarrow 1$ such that $\operatorname{hdim}^{G^{\prime}}\left(\underline{E} G^{\prime}\right) \leq 1$ and $A^{\prime} \subseteq A$ is a subgroup of finite index. We have $A^{\prime}=\bigcup_{i \geq 0}\left(A^{\prime} \cap H_{1}\left(V_{i}\right)\right)$. The computations appearing in the proof above imply that either $A^{\prime}$ is a finite abelian group or $H^{1}\left(A^{\prime} ; \mathbb{Q}\left[A^{\prime}\right]\right) \cong$ $\operatorname{coker}\left(\delta_{\mathbb{Q}\left[A^{\prime}\right]}\right) \neq 0$, and that $H^{k}\left(A^{\prime} ; \mathbb{Q}\left[A^{\prime}\right]\right)=0$ for $k \geq 2$. From the HochschildSerre cohomology spectral sequence applied to $1 \rightarrow C \rightarrow G^{\prime} \rightarrow A^{\prime} \rightarrow 1$ we obtain an isomorphism $H^{1}\left(A^{\prime} ; \mathbb{Q}\left[A^{\prime}\right]\right) \cong H^{2}\left(G^{\prime} ; \mathbb{Q}\left[A^{\prime}\right]\right)$, and the latter term vanishes as $\operatorname{hdim}^{G / K}(\underline{E}(G / K)) \leq 1$. We conclude that $A^{\prime}$ and hence $A$ are finite, showing that $G$ is virtually cyclic. This finishes the proof of assertion (i).

(ii) First we show that $\operatorname{hdim}^{G}(\underline{\underline{E}} G) \leq 2$ provided that $\operatorname{hdim}^{G}(\underline{E} G) \leq 1$ and $G$ is countable. Consider an infinite virtually cyclic subgroup $V \subseteq G$. Let $H \subseteq N_{G}[V]$ be a finitely generated subgroup such that $V \subseteq H$. From $\operatorname{hdim}^{G}(\underline{E} G) \leq 1$ we get $\operatorname{hdim}^{H}(\underline{E} H) \leq 1$, and since $H$ is finitely generated, this implies that it is virtually finitely generated free (see [11, Theorem 1]). In particular, $H$ is word-hyperbolic, so we conclude from Theorem 3.1 and Example 3.6 that $N_{H}[V]$ is virtually cyclic. Since $H=H \cap N_{G}[V]=N_{H}[V]$, this means that $H$ is virtually cyclic. Thus, we have shown that $N_{G}[V]$ is locally virtually cyclic, and as $\operatorname{hdim}^{G}(\underline{E} G) \leq 1$ implies $\operatorname{hdim}^{N_{G}[V]}\left(\underline{E} N_{G}[V]\right) \leq 1$, assertion (i) shows that $N_{G}[V]$ is locally finite or virtually cyclic. In fact, $N_{G}[V]$ contains the infinite virtually cyclic group $V$, so $N_{G}[V]$ is virtually cyclic. This implies that $\mathcal{V C Y}[V]$ consists of all subgroups of $N_{G}[V]$ and, hence, $\{\bullet\}$ is a model for $E_{\mathcal{V C Y}[V]}\left(N_{G}[V]\right)$. Summarizing, we can arrange that $\operatorname{dim}\left(\underline{E} N_{G}[V]\right) \leq 1$ and $\operatorname{dim}\left(E_{\mathcal{V C Y}[V]}\left(N_{G}[V]\right)\right) \leq 1$ for every infinite virtually cyclic subgroup $V$ of $G$. Now Theorem 2.3 together with Remark 2.5 implies $\operatorname{hdim}^{G}(\underline{\underline{E}} G) \leq 2$.

If $G$ is countable and locally virtually cyclic, then $\operatorname{hdim}^{G}(\underline{\underline{E}} G) \leq 1$ has already been proved in assertion (i). It remains to show that $G$ is locally virtually cyclic provided that $\operatorname{hdim}^{G}(\underline{E} G)$ and $\operatorname{hdim}^{G}(\underline{\underline{E}} G)$ are less or equal to 1 . Let $G_{0} \subseteq G$ be a finitely generated subgroup of $G$. Then $\operatorname{hdim}^{G}\left(\underline{E} G_{0}\right)$ and $\operatorname{hdim}^{G}\left(\underline{\underline{E}} G_{0}\right)$ are less or equal to 1 . This implies that $G_{0}$ contains a finitely generated free subgroup $G_{1}$ of finite index (see [11, Theorem 1]). The group $G_{1}$ satisfies $N M_{\mathcal{F} \mathcal{I N} \subseteq \mathcal{V} \mathcal{Y} Y}$ by 
Example 3.6, so from Corollary 2.11 we get a cellular $G_{1}$-pushout

$$
\begin{aligned}
& \coprod_{V \in \mathcal{M}} G_{1} \times_{V} \underline{E} V \longrightarrow \underline{E} G_{1} \\
& \downarrow \amalg_{V \in \mathcal{M}} \operatorname{id}_{G_{1}} \times_{V} i_{V} \downarrow \\
& \amalg_{V \in \mathcal{M}} G_{1} \stackrel{\vee}{\times}_{V} \operatorname{cone}(\underline{E} V) \longrightarrow \stackrel{\sim}{E} G_{1}
\end{aligned}
$$

where $\mathcal{M}$ is a complete system of representatives of the conjugacy classes of maximal infinite virtually cyclic subgroups of $G_{1}$ and $i_{V}$ is the obvious inclusion. Dividing out the $G_{1}$-action and then taking the associated Mayer-Vietoris exact sequence yields an injection

$$
\bigoplus_{V \in \mathcal{M}} H_{1}(V \backslash \underline{E} V) \rightarrow H_{1}\left(G_{1} \backslash \underline{E} G_{1}\right)
$$

because $\operatorname{hdim}^{G_{1}}\left(\underline{\underline{E}} G_{1}\right) \leq 1$. Since $G_{1}$ is finitely generated free, $H_{1}\left(G_{1} \backslash \underline{E} G_{1}\right)$ is finitely generated free and $H_{1}(V \backslash \underline{E} V)$ is isomorphic to $\mathbb{Z}$ for every $V \in \mathcal{M}$. This implies that $\mathcal{M}$ is finite, i.e., $G_{1}$ contains only finitely many infinite cyclic subgroups up to conjugacy. Thus, $G_{1}$ is either trivial or infinite cyclic, showing that $G_{0}$ is virtually cyclic. Hence $G$ is locally virtually cyclic. This finishes the proof of Theorem 5.33.

Example 5.34. For the prime number $p$, let $\mathbb{Z}[1 / p]$ be the subgroup of $\mathbb{Q}$ consisting of rational numbers $x \in \mathbb{Q}$ for which $p^{n} \cdot x \in \mathbb{Z}$ for some positive integer $n$ (see Example 2.6). Every finitely generated subgroup of $\mathbb{Z}[1 / p]$ is trivial or infinite cyclic. Thus, Theorem 5.33 implies

$$
\begin{gathered}
\operatorname{hdim}^{\mathbb{Z}[1 / p]}(\underline{E} \mathbb{Z}[1 / p])=2 ; \\
\operatorname{hdim}^{\mathbb{Z}[1 / p]}(\underline{\underline{E}} \mathbb{Z}[1 / p])=1 .
\end{gathered}
$$

Remark 5.35. Let $G$ be a countable group. Then Theorem 5.33 implies that $G$ is infinite locally finite if and only if $\operatorname{hdim}^{G}(\underline{E} G)=\operatorname{hdim}^{G}(\underline{\underline{E}} G)=1$ holds.

\section{Equivariant homology of relative assembly maps}

Let $\mathcal{H}_{*}^{?}$ be an equivariant homology theory in the sense of $[14$, Section 1$]$. Our main example is the equivariant homology theory $H_{*}^{?}\left(-; \mathbf{K}_{R}\right)$ appearing in the 
$K$-theoretic Farrell-Jones conjecture, where $R$ is a ring (here, rings are always assumed to be associative and unital). It has the property that

$$
H_{n}^{G}\left(G / H ; \mathbf{K}_{R}\right)=H_{n}^{H}\left(\{\bullet\} ; \mathbf{K}_{R}\right)=K_{n}(R H)
$$

holds for every subgroup $H \subseteq G$, where $K_{n}(R H)$ denotes the algebraic $K$-theory of the group ring $R H$. The Farrell-Jones conjecture for a group $G$ and a ring $R$ says that the assembly map, which is the map induced by the projection $\underline{\underline{E}} G \rightarrow G / G$, is an isomorphism

$$
H_{n}^{G}\left(\underline{\underline{E}} G ; \mathbf{K}_{R}\right) \stackrel{\cong}{\rightrightarrows} H_{n}^{G}\left(G / G ; \mathbf{K}_{R}\right)=K_{n}(R G)
$$

for all $n \in \mathbb{Z}$.

Bartels [2] has shown that for every group $G$, every $\operatorname{ring} R$, and every $n \in \mathbb{Z}$ the relative assembly map

$$
H_{n}^{G}\left(\underline{E} G ; \mathbf{K}_{R}\right) \rightarrow H_{n}^{G}\left(\underline{\underline{E}} G ; \mathbf{K}_{R}\right)
$$

is split-injective. Hence, the source of the assembly map appearing in the FarrellJones conjecture can be computed in two steps, involving the computation of $H_{n}^{G}\left(\underline{E} G ; \mathbf{K}_{R}\right)$ and the computation of the remaining term which we denote by $H_{n}^{G}\left(\underline{E} G \rightarrow \underline{\underline{E}} G ; \mathbf{K}_{R}\right)$. Rationally, $H_{n}^{G}\left(\underline{E} G ; \mathbf{K}_{R}\right)$ can be computed using equivariant Chern characters (see [14, Section 1]) or with the help of nice models for $\underline{E} G$.

The goal of this section is to give some information about $H_{n}^{G}\left(\underline{E} G \rightarrow \underline{\underline{E}} G ; \mathbf{K}_{R}\right)$. Namely, using the induction structure on $\mathcal{H}_{*}$, Corollary 2.10 implies:

Corollary 6.1. Let $\mathcal{H}_{*}^{\text {? }}$ be an equivariant homology theory. Suppose that the group $G$ satisfies $\left(M_{\mathcal{F} \mathcal{I N} \subseteq \mathcal{V} C \mathcal{Y}}\right)$. Let $\mathcal{M}$ be a complete system of representatives of the conjugacy classes of maximal infinite virtually cyclic subgroups of $G$. Then, for $n \in \mathbb{Z}$, we obtain an isomorphism

$$
\bigoplus_{V \in \mathcal{M}} \mathcal{H}_{n}^{N_{G} V}\left(\underline{E} N_{G} V \rightarrow E W_{G} V\right) \stackrel{\cong}{\rightarrow} \mathcal{H}_{n}^{G}(\underline{E} G \rightarrow \underline{\underline{E}} G)
$$

Remark 6.2. The term $\mathcal{H}_{n}^{N_{G} V}\left(\underline{E} N_{G} V \rightarrow E W_{G} V\right)$ appearing in Corollary 6.1 can be analyzed further. 
Namely, by assigning to a free $W_{G} V$-CW-complex $X$ the $\mathbb{Z}$-graded abelian group $\mathcal{H}_{*}^{N_{G} V}\left(\underline{E} N_{G} V \times X \rightarrow X\right)$, we obtain a $W_{G} V$-homology theory for free $W_{G} V$-CW-complexes. Here, we consider $X$ as an $N_{G} V$ - $C W$-complex by restriction with the projection $N_{G} V \rightarrow W_{G} V$, equip $\underline{E} N_{G} V \times X$ with the diagonal $N_{G} V$-action and let $\underline{E} N_{G} V \times X \rightarrow X$ be the projection onto $X$. There is an Atiyah-Hirzebruch spectral sequence converging to $\mathcal{H}_{p+q}^{N_{G} V}\left(\underline{E} N_{G} V \times X \rightarrow X\right)$ whose $E^{2}$-term is

$$
E_{p, q}^{2}=H_{p}^{W_{G} V}\left(E W_{G} V ; \mathcal{H}_{q}^{N_{G} V}\left(E N_{G} V \times W_{G} V \rightarrow W_{G} V\right)\right)
$$

where the right $\mathbb{Z}\left[W_{G} V\right]$-module structure on $\mathcal{H}_{q}^{N_{G} V}\left(\underline{E} N_{G} V \times W_{G} V \rightarrow W_{G} V\right)$ comes from the obvious right $W_{G} V$-action on $W_{G} V$ and the trivial $W_{G} V$-action on $\underline{E} N_{G} V$.

For any $N_{G} V$-space $Y$, the map $N_{G} V \times \times_{V} \operatorname{res}_{N_{G} V}^{V} Y \rightarrow Y \times W_{G} V$ given by sending $(g, y)$ to $(g y, g V)$ is an $N_{G} V$-homeomorphism. In particular, since $\operatorname{res}_{N_{G} V}^{V} \underline{E} N_{G} V$ is a model for $\underline{E} V$, we obtain an $N_{G} V$-homeomorphism $N_{G} V \times_{V}$ $\underline{E} V \rightarrow \underline{E} N_{G} V \times W_{G} V$. Together with the induction structure, this induces an isomorphism of abelian groups

$$
\left.\mathcal{H}_{q}^{N_{G} V}\left(E N_{G} V \times W_{G} V \rightarrow W_{G} V\right)\right) \cong \mathcal{H}_{q}^{V}(\underline{E} V \rightarrow\{\bullet\}),
$$

which becomes an isomorphism of $\mathbb{Z}\left[N_{G} V\right]$-modules if we equip the target with the following $W_{G} V$-action.

Given $\bar{g} \in W_{G} V$, choose a preimage $g \in N_{G} V$ under the projection $N_{G} V \rightarrow$ $W_{G} V$. Conjugation with $g$ yields a group homomorphism $c(g): V \rightarrow V$. The induction structure yields an isomorphism $\mathcal{H}_{q}^{V}(\underline{E} V \rightarrow\{\bullet\}) \rightarrow \mathcal{H}_{q}^{V}\left(\operatorname{ind}_{c(g)} \underline{E} V \rightarrow\right.$ $\left.\operatorname{ind}_{c(g)}\{\bullet\}\right)$. Let $f: \operatorname{ind}_{c(g)} \underline{E} V \rightarrow \underline{E} V$ be the $V$-homotopy equivalence which is unique up to $V$-homotopy. Obviously, $\operatorname{ind}_{c(g)}\{\bullet\}=\{\bullet\}$. Hence, $f$ induces a homomorphism $f_{q}: \mathcal{H}_{q}^{V}\left(\operatorname{ind}_{c(g)} \underline{E} V \rightarrow \operatorname{ind}_{c(g)}\{\bullet\}\right) \rightarrow \mathcal{H}_{q}^{V}(\underline{E} V \rightarrow\{\bullet\})$. The composition of these two homomorphisms is an automorphism of $\mathcal{H}_{q}^{V}(\underline{E} V \rightarrow\{\bullet\})$, which we define to be multiplication with $\bar{g}$. It is easy to check that this definition is independent of the choice of the preimage $g$ of $\bar{g}$, and that it defines a $W_{G} V$ action on $\mathcal{H}_{q}^{V}(\underline{E} V \rightarrow\{\bullet\})$ which is compatible with the one on $\mathcal{H}_{q}^{N_{G} V}\left(E N_{G} V \times\right.$ $\left.W_{G} V \rightarrow W_{G} V\right)$. 
Example 6.3. Let us consider the special case that the equivariant homology theory is $H_{*}^{?}\left(-; \mathbf{K}_{R}\right)$, the one appearing in the $K$-theoretic Farrell-Jones conjecture. Let $V$ be an infinite virtually cyclic group. If $V$ is of type $I$, we can write $V$ as a semi-direct product $F \rtimes \mathbb{Z}$, and $H_{*}^{V}\left(\underline{E} V \rightarrow\{\bullet\} ; \mathbf{K}_{R}\right)$ is the non-connective version of Waldhausen's Nil-term associated to this semi-direct product (see $[1$, Section 9]). If $V$ is of type II, then it can be written as an amalgamated product $V_{1} * V_{0} V_{2}$ of finite groups, where $V_{0}$ has index two in both $V_{1}$ and $V_{2}$. In this case, $H_{*}^{V}\left(\underline{E} V \rightarrow\{\bullet\} ; \mathbf{K}_{R}\right)$ is the non-connective version of Waldhausen's Nil-term associated to this amalgamated product (see [1, Section 9]). The $W_{G} V$-action on these Nil-terms comes from the action of $N_{G} V$ on $V$ by conjugation and the fact that inner automorphisms of groups induce the identity on algebraic $K$-groups associated to group rings.

In the case of $L$-theory, the terms $H_{*}^{V}\left(\underline{E} V \rightarrow\{\bullet\} ; \mathbf{L}_{R}^{\langle-\infty\rangle}\right)$ vanish if $V$ is of type I and are given by UNil-terms if $V$ is of type II (see [15, Lemma 4.2]).

Example 6.4. Let $\mathcal{H}_{*}^{\text {? }}$ be an equivariant homology theory. Suppose that the group $G$ satisfies $\left(N M_{\mathcal{F} \mathcal{I N} \subseteq \mathcal{V} \mathcal{Y} Y}\right)$. Let $\mathcal{M}$ be a complete system of representatives of the conjugacy classes of maximal infinite virtually cyclic subgroups of $G$. Then, for $n \in \mathbb{Z}$, Corollary 6.1 yields an isomorphism

$$
\bigoplus_{V \in \mathcal{M}} \mathcal{H}_{n}^{V}(\underline{E} V \rightarrow\{\bullet\}) \cong \mathcal{H}_{n}^{G}(\underline{E} G \rightarrow \underline{\underline{E}} G) .
$$

Now assume in addition that $G$ is torsionfree. If the equivariant homology theory is $H_{*}^{?}\left(-, \mathbf{K}_{R}\right)$, then $H_{*}^{V}\left(\underline{E} V \rightarrow\{\bullet\} ; \mathbf{K}_{R}\right)$ reduces to $N K_{n}(R) \oplus N K_{n}(R)$. Here, $N K_{n}(R)$ is the $n$-th Bass-Nil-group which is defined as the cokernel of the obvious split injection $K_{n}(R) \rightarrow K_{n}(R[t])$. In the case of $L$-theory, we get $H_{*}^{V}(\underline{E} V \rightarrow$ $\left.\{\bullet\} ; \mathbf{L}_{R}^{\langle-\infty\rangle}\right)=0$.

\section{References}

[1] A. Bartels and W. Lück. Isomorphism conjecture for homotopy $K$-theory and groups acting on trees. J. Pure Appl. Algebra, 205(3):660-696, 2006.

[2] A. C. Bartels. On the domain of the assembly map in algebraic $K$-theory. Algebr. Geom. Topol., 3:1037-1050 (electronic), 2003. 
[3] M. R. Bridson and A. Haefliger. Metric spaces of non-positive curvature. Springer-Verlag, Berlin, 1999. Die Grundlehren der mathematischen Wissenschaften, Band 319 .

[4] K. S. Brown. Cohomology of groups, volume 87 of Graduate Texts in Mathematics. Springer-Verlag, New York, 1982.

[5] F. X. Connolly, B. Fehrmann, and M. Hartglass. On the dimension of the virtually cyclic classifying space of a crystallographic group. arXiv: math.AT/0610387, 2006.

[6] J. F. Davis and W. Lück. Spaces over a category and assembly maps in isomorphism conjectures in $K$ - and $L$-theory. $K$-Theory, 15(3):201-252, 1998.

[7] J. F. Davis and W. Lück. The p-chain spectral sequence. K-Theory, 30(1):71-104, 2003. Special issue in honor of Hyman Bass on his seventieth birthday. Part I.

[8] W. Dicks, P. H. Kropholler, I. J. Leary, and S. Thomas. Classifying spaces for proper actions of locally finite groups. J. Group Theory, 5(4):453-480, 2002 .

[9] M. J. Dunwoody. Accessibility and groups of cohomological dimension one. Proc. London Math. Soc. (3), 38(2):193-215, 1979.

[10] D. Juan-Pineda and I. J. Leary. On classifying spaces for the family of virtually cyclic subgroups. In Recent developments in algebraic topology, volume 407 of Contemp. Math., pages 135-145. Amer. Math. Soc., Providence, RI, 2006 .

[11] A. Karrass, A. Pietrowski, and D. Solitar. Finite and infinite cyclic extensions of free groups. J. Austral. Math. Soc., 16:458-466, 1973. Collection of articles dedicated to the memory of Hanna Neumann, IV.

[12] P. H. Kropholler and S. Thomas. The consistency of Holt's conjectures on cohomological dimension of locally finite groups. J. London Math. Soc. (2), 55(1):76-86, 1997. 
[13] W. Lück. Transformation groups and algebraic K-theory. Springer-Verlag, Berlin, 1989.

[14] W. Lück. Chern characters for proper equivariant homology theories and applications to $K$ - and L-theory. J. Reine Angew. Math., 543:193-234, 2002.

[15] W. Lück. $K$ - and $L$-theory of the semi-direct product of the discrete 3 dimensional Heisenberg group by $\mathbb{Z} / 4$. Geom. Topol., 9:1639-1676 (electronic), 2005.

[16] W. Lück. Survey on classifying spaces for families of subgroups. In Infinite groups: geometric, combinatorial and dynamical aspects, volume 248 of Progr. Math., pages 269-322. Birkhäuser, Basel, 2005.

[17] J.-P. Serre. Trees. Springer-Verlag, Berlin, 1980. Translated from the French by J. Stillwell.

[18] J. R. Stallings. On torsion-free groups with infinitely many ends. Annals of Math. (2), 88:312-334, 1968.

[19] R. G. Swan. Groups of cohomological dimension one. J. Algebra, 12:585-610, 1969.

Wolfgang Lück

Fachbereich Mathematik

Universität Münster

Einsteinstr. 62

48149 Münster

Germany

E-mail: lueck@math.uni-muenster.de

www: http://www.math.uni-muenster.de/u/lueck/

FAX: 492518338370

Michael Weiermann

Fachbereich Mathematik

Universität Münster 
On The Classifying Space of The Family of Virtually Cyclic Subgroups 555

Einsteinstr. 62

48149 Münster

Germany

E-mail: michi@uni-muenster.de 